\title{
A CLASS OF NONHOLOMORPHIC MODULAR FORMS II: EQUIVARIANT ITERATED EISENSTEIN INTEGRALS
}

\author{
FRANCIS BROWN \\ Mathematical Institute, University of Oxford; \\ email: francis.brown@all-souls.ox.ac.uk
}

Received 26 April 2019; accepted 14 April 2020

\begin{abstract}
We introduce a new family of real-analytic modular forms on the upper-half plane. They are arguably the simplest class of 'mixed' versions of modular forms of level one and are constructed out of real and imaginary parts of iterated integrals of holomorphic Eisenstein series. They form an algebra of functions satisfying many properties analogous to classical holomorphic modular forms. In particular, they admit expansions in $q, \bar{q}$ and $\log |q|$ involving only rational numbers and singlevalued multiple zeta values. The first nontrivial functions in this class are real-analytic Eisenstein series.
\end{abstract}

2010 Mathematics Subject Classification: 11F03, 11M32 (primary); 11F67, 11M3, 81T30 (secondary)

\section{Introduction}

Let $\mathfrak{H}=\{z: \operatorname{Im} z>0\}$ be the upper-half plane with its action of $\mathrm{SL}_{2}(\mathbb{Z})$ :

$$
\gamma z=\frac{a z+b}{c z+d}, \quad \text { where } \gamma=\left(\begin{array}{ll}
a & b \\
c & d
\end{array}\right) \in \mathrm{SL}_{2}(\mathbb{Z}) .
$$

We shall say that a real-analytic function

$$
f: \mathfrak{H} \longrightarrow \mathbb{C}
$$

is modular of weights $(r, s)$ with $r, s \in \mathbb{Z}$ if for all $\gamma \in \mathrm{SL}_{2}(\mathbb{Z})$, it satisfies

$$
f(\gamma z)=(c z+d)^{r}(c \bar{z}+d)^{s} f(z) .
$$

(c) The Author 2020. This is an Open Access article, distributed under the terms of the Creative Commons Attribution licence (http://creativecommons.org/licenses/by/4.0/), which permits unrestricted re-use, distribution, and reproduction in any medium, provided the original work is properly cited. 
In this paper, we construct a space $\mathcal{M I}^{E}$ of real-analytic modular functions on $\mathfrak{H}$, which are modular analogues of the single-valued polylogarithms. They are obtained by taking real and imaginary parts of iterated primitives of the holomorphic Eisenstein series, which are defined for all even $k \geqslant 4$ by

$$
\mathbb{G}_{k}(q)=-\frac{B_{k}}{2 k}+\sum_{n \geqslant 1} \sigma_{k-1}(n) q^{n},
$$

where $\sigma$ denotes the divisor function. The modular analogues of the single-valued logarithm $\log |z|^{2}$ are real-analytic Eisenstein series and are well-known. The modular analogues of the Bloch-Wigner dilogarithm $D(z)=\operatorname{Im}\left(\operatorname{Li}_{2}(z)+\log |z| \log (1-z)\right)$ have already led to completely new functions with interesting properties [6]. In this paper, we study the entire space of such functions. Most of our results are summarized here.

THEOREM 1.1. The space of real-analytic functions $\mathcal{M I}^{E}$ has the following properties:

(1) (Expansions). Every $f \in \mathcal{M I}^{E}$ is modular of weights $(r, s)$ for integers $r, s \geqslant 0$. It admits a unique expansion, for some $N \geqslant 0$, of the form

$$
f(q)=\sum_{k=-N}^{N} \mathbb{L}^{k}\left(\sum_{m, n \geqslant 0} a_{m, n}^{(k)} q^{m} \bar{q}^{n}\right),
$$

where $q=e^{2 \pi i z}, \mathbb{L}:=\log |q|=-2 \pi \operatorname{Im}(z)$, and the coefficients $a_{m, n}^{(k)}$ are single-valued multiple zeta values.

(2) (Filtrations). The space $\mathcal{M} \mathcal{I}^{E}\left[\mathbb{L}, \mathbb{L}^{-1}\right]$ is an algebra over $\mathbb{Q}$, bigraded by the modular weights $(r, s)$. Furthermore, $\mathcal{M I}^{E}$ admits a filtration by length

$$
0 \subset \mathcal{M I}_{0}^{E} \subset \mathcal{M I}_{1}^{E} \subset \cdots \subset \mathcal{M I}_{k}^{E} \subset \cdots
$$

and also by motivic weight $M$, which is conjecturally a grading. (In this paper, we have chosen to halve the M-weight compared to [6], in keeping with the standard convention for the weight of multiple zeta values, which is one half of the Hodge-theoretic weight.) The coefficients in the expansion (1.4) have $M$-weight bounded above by the $M$-weight of $f$, where $\mathbb{L}$ has $M$-weight 1 .

(3) (Finiteness). The subspaces $M_{w} \mathcal{M I}_{r, s}^{E}$ of motivic weight $\leqslant w$, and fixed modular degree $(r, s)$, are finite-dimensional $\mathbb{Q}$-vector spaces. A function in such a subspace is uniquely determined by a finite number of coefficients $a_{m, n}^{(k)}$. 
(4) (Differential structure). Let $\partial$ be the differential operator of modular bidegree $(1,-1)$, which acts on functions of modular weights $(r, \bullet)$ by

$$
\partial_{r}=(z-\bar{z}) \frac{\partial}{\partial z}+r .
$$

Let $\bar{\partial}$ denote its complex conjugate of modular bidegree $(-1,1)$. These operators are close variants of the Maass raising and lowering operators. Then

$$
\begin{aligned}
& \partial \mathcal{M I}_{\ell}^{E} \subset \mathcal{M I}_{\ell}^{E}+\left(E \times \mathcal{M I}_{\ell-1}^{E}\right), \\
& \bar{\partial} \mathcal{M I}_{\ell}^{E} \subset \mathcal{M I}_{\ell}^{E}+\left(\bar{E} \times \mathcal{M I}_{\ell-1}^{E}\right),
\end{aligned}
$$

where $E$ denotes the $\mathbb{Q}$-vector space generated by the product of a holomorphic Eisenstein series (1.3) with a non-negative power of $\mathbb{L}$. The terms on both sides of these equations are contained in the space of realanalytic functions on the upper-half plane. Concretely, the first equation states that if $f \in \mathcal{M I}_{\ell}^{E}$, we may write

$$
\partial f=f_{0}+\sum_{i \geqslant 2, j \geqslant 0} \mathbb{G}_{2 i} \mathbb{L}^{j} f_{i, j}
$$

where $f_{0} \in \mathcal{M I}_{\ell}^{E}$ and $f_{i, j} \in \mathcal{M I}_{\ell-1}^{E}$. The functions $f_{0}, f_{i, j}$ are in fact unique. In particular, for $f \in \mathcal{M I}_{\ell}^{E}$ of length $\ell$ and of modular weights $(n, 0)$, we have

$$
\partial f \in E \times \mathcal{M I}_{\ell-1}^{E}
$$

since the modular weights of $\mathcal{M I}^{E}$ lie in the first quadrant. In this case, the function $f_{0}$ vanishes in equation (1.6) and $\partial f$ is built out of functions of strictly smaller length. Therefore the space $\mathcal{M I}^{E}$ is generated by taking iterated primitives (or antiderivatives) of holomorphic and antiholomorphic Eisenstein series with respect to $\partial, \bar{\partial}$. Equations (1.5) also imply eigenvector relations on $\mathcal{M I}^{E}$ with respect to the bigraded Laplace-Beltrami operator.

(5) (Orthogonality). For every $f \in \mathcal{M I}^{E}$, the function $\partial f$ is orthogonal to the space of holomorphic cusp forms with respect to the Petersson inner product.

(6) (Algebraic structure). Let $\operatorname{lw}\left(\mathcal{M I}^{E}\right) \subset \mathcal{M I}^{E}$ denote the subspace of functions with modular weights $(n, 0)$, that is, satisfying the classical modular transformation property with no antiholomorphic automorphy factor (the notation lw stands for 'lowest weight' in the sense of $\mathfrak{s l}_{2}$ representations). Then $\operatorname{lw}\left(\mathcal{M I}^{E}\right)$ is dual to the lowest weight vectors in 
a certain well-known Lie algebra $\mathfrak{u}^{\text {geom }}$ of geometric derivations on the free Lie algebra on two generators.

This theorem is not exhaustive. The class of functions $\mathcal{M I}^{E}$ has several other properties which are proved in this paper, and many more which are not. For example, with every $f \in \mathcal{M I}^{E}$, we can associate an $L$-function that is a linear combination of Dirichlet series. It admits a meromorphic continuation to $\mathbb{C}$ and satisfies a functional equation. We show that this $L$-function is a single-variable restriction of the multiple-variable $L$-functions recently defined in [1].

\subsection{Examples}

1.1.1. Length zero. In length zero, we have

$$
\mathcal{M I}_{0}^{E}=\mathcal{Z}^{\mathrm{sv}}
$$

where $\mathcal{Z}^{\text {sv }}$ is the weight-filtered $\mathbb{Q}$-algebra of single-valued multiple zeta values, viewed as constant functions of modular weights $(0,0)$. A definition is recalled below. This space is already infinite-dimensional but is finite in each $M$-weight. It does not contain any powers of $\pi$ but contains the odd zeta values

$$
\zeta^{\mathrm{sv}}(2 n+1)=2 \zeta(2 n+1)
$$

in $M$-weight $2 n+1$, for $n \geqslant 1$. The first interesting generator occurs in weight 11 :

$$
\zeta^{\mathrm{sv}}(3,5,3)=2 \zeta(3,5,3)-2 \zeta(3) \zeta(3,5)-10 \zeta(3)^{2} \zeta(5) .
$$

The space $\mathcal{M I}^{E}$ is an algebra over $\mathcal{Z}^{\text {sv }}$ and satisfies

$$
M_{0} \mathcal{M I}_{0}^{E}=M_{0} \mathcal{Z}^{\mathrm{sv}}=\mathbb{Q}
$$

1.1.2. Length one. In length one, $\mathcal{M I}^{E}$ contains the first interesting functions:

$$
\mathcal{M I}_{1}^{E} \cong \bigoplus_{r, s} \mathcal{E}_{r, s} \mathcal{Z}^{\mathrm{sv}}
$$

where the direct sum is over all integers $r, s \geqslant 0$ such that $w=r+s>0$ is even and

$$
\mathcal{E}_{r, s}(z)=\frac{w !}{(2 \pi i)^{w+2}} \frac{1}{2} \sum_{(m, n) \in \mathbb{Z}^{2} \backslash(0,0)} \frac{\mathbb{L}}{(m z+n)^{r+1}(m \bar{z}+n)^{s+1}}
$$


are real-analytic Eisenstein series, which are modular of weights $(r, s)$. The functions $\mathcal{E}_{r, s}$ admit an expansion of the form

$$
\mathcal{E}_{r, s}=a_{w} \mathbb{L}+b_{r, s} \zeta^{\mathrm{sv}}(w+1) \mathbb{L}^{-w}+\left(\text { a series in } \mathbb{Q}[[q, \bar{q}]]\left[\mathbb{L}^{-1}\right]\right),
$$

where $a_{w}, b_{r, s}$ are explicit rational numbers. The elements $\mathcal{E}_{r, s}$ and $\mathbb{L}$ have $M$ filtration equal to 1 . One checks that the $M$-filtration of every term in the previous expansion is indeed $\leqslant 1$. The differential structure amounts to the equations

$$
\text { ว } \mathcal{E}_{w, 0}=\mathbb{L} \mathbb{G}_{w+2} \quad \text { and } \quad \partial \mathcal{E}_{r, s}=(r+1) \mathcal{E}_{r+1, s-1}
$$

for all $1 \leqslant s \leqslant w$, and their complex conjugates. The family of functions $\mathcal{E}_{r, s}$ is associated with a universal mixed elliptic motive [21] whose fibre at the cusp is a simple extension of mixed Tate motives: namely, $\mathbb{Q}(-w-1)$ by $\mathbb{Q}$, whose (single-valued) periods are rational multiples of 1 and $\zeta^{\text {sv }}(w+1)$. The completed $L$-function $\Lambda\left(\mathcal{E}_{a, b}, \bullet\right)$ associated with $\mathcal{E}_{a, b}$ is proportional to a certain rational function multiplied by a product of two Riemann zeta functions $\zeta(\bullet) \zeta(\bullet-2 w+1)$.

1.1.3. Higher length. Starting from length two, $\mathcal{M I}^{E}$ contains polynomials in the $\mathcal{E}_{r, s}$ together with a large array of completely new functions, some examples of which were described in [6]. In length two, the enumeration is as follows. To every pair of Eisenstein series $\mathbb{G}_{2 m+2}, \mathbb{G}_{2 n+2}$ we can take real and imaginary parts of the double iterated integrals of $\mathbb{G}_{2 m+2} \tau^{i}, \mathbb{G}_{2 n+2} \tau^{j}$ for $0 \leqslant i \leqslant 2 m, 0 \leqslant j \leqslant 2 n$ in a prescribed way to obtain a priori $(2 m+1)(2 n+1)$ functions. However, the condition of orthogonality (5) imposes a constraint (in every relevant modular bidegree) for every holomorphic cusp form and pushes the dimension of $\mathcal{M I}_{2}^{E}$ down by that amount. The precise condition involves the critical values of $L$ functions of cusp forms, and is related to the existence of nontrivial extensions of $\mathbb{Q}$ by motives of cusp forms (which are governed by noncritical values of the said $L$-functions). Thus the space of functions $\mathcal{M I}^{E}$ grows approximately quadratically in length two and cubically in length three. The precise enumeration in lengths two and three is stated in Section 12.4, but is not known explicitly in length four or above, and is closely related to deep questions about the structure of the category of mixed elliptic motives, the Broadhurst-Kreimer conjecture on depth-graded multiple zeta values, and the existence of 'higher' Rankin-Selberg convolutions.

\subsection{Comments}

- The class of functions $\mathcal{M I}^{E}$ shares many properties with classical holomorphic modular forms. Indeed, a key feature of the latter is the fact that a holomorphic 
modular form is uniquely determined by an explicit number of Fourier coefficients depending on its modular weight. The same property is true for the subspace of functions in $\mathcal{M I}^{E}$ of bounded motivic weight. It would be very interesting to make this effective and determine an exact bound for the number of expansion coefficients that determine an element of $\mathcal{M I}^{E}$ uniquely.

- It should be relatively straightforward to generalize this theory to higher levels. In a different direction, $\mathcal{M I}^{E}$ is a subspace of a larger class of functions, which also includes iterated primitives of cusp forms as well as Eisenstein series [2]. For this, one must allow poles at the cusps, for example. The length one part of this class is the theory of weak harmonic Maass forms and mock modular forms of level one [7], but involves new functions thereafter.

- The algebra $\mathfrak{u}^{\text {geom }}$ has occurred in various guises, most notably as the fundamental representation of the Tannaka Lie algebra of Hain and Matsumoto's universal mixed elliptic motives. The space $\mathcal{M I}^{E}$ can be viewed as an elementary realization of this category as a space of functions: for example, the structure of $\mathcal{M} \mathcal{I}^{E}$ encodes information about extension data in that category.

- The functions $\mathcal{M I}^{E}$ are expected to contain the modular graph functions studied in string theory (see below), and in fact general correlation functions for configurations of points on the universal elliptic curve. If true, then Theorem 1.1 implies most of the open conjectures about this class of functions.

- The Lie algebra $\mathfrak{u}^{\text {geom }}$ is closely related to combinatorial relations satisfied by multiple zeta values. In fact, in an earlier work, we showed that it satisfies linearized versions of the double-shuffle equations, about which several important conjectures are still open. Items (5) and (6) taken together therefore imply that these equations are, in a precise sense, orthogonal to cusp forms. This should shed light on their structure.

1.3. Construction. The functions in $\mathcal{M I}^{E}$ are the equivariant sections of the unipotent fundamental group of the universal elliptic curve. To explain this, let us recall the construction of single-valued polylogarithms in genus zero [4], before passing to its elliptic generalization. Consider the following multivalued function $L(z)$ on $\mathbb{C}^{\times} \backslash\{1\}$ taking values in formal power series $\mathbb{C}\left\langle\left\langle\mathrm{x}_{0}, \mathrm{x}_{1}\right\rangle\right\rangle$ in two noncommuting variables $x_{0}, x_{1}$. It is the unique solution to the KnizhnikZamolodchikov equation

$$
d L(z)=\omega_{K Z} L(z), \quad \text { where } \omega_{K Z}=\frac{d z}{z} \mathrm{x}_{0}+\frac{d z}{1-z} \mathrm{x}_{1},
$$


with the initial condition $L\left(\overrightarrow{1}_{0}\right)=1$ (denoting the regularized limit as $z \rightarrow 0$ along the real axis with unit speed), where $\mathrm{x}_{0}, \mathrm{x}_{1}$ act by left multiplication. It has a canonical single-valued version $\mathcal{L}: \mathbb{C}^{\times} \backslash\{1\} \rightarrow \mathbb{C}\left\langle\left\langle\mathrm{X}_{0}, \mathrm{X}_{1}\right\rangle\right\rangle$, which satisfies the identical differential equation and initial condition. It can be viewed as a formal power series

$$
\mathcal{L}=\sum_{w} \mathcal{L}_{w}(z) w \in \mathbb{C}\left\langle\left\langle\mathrm{x}_{0}, \mathrm{x}_{1}\right\rangle\right\rangle
$$

where the sum ranges over all words $w$ in $\mathrm{x}_{0}, \mathrm{x}_{1}$. Its coefficients $\mathcal{L}_{w}(z)$ are singlevalued analogues of multiple polylogarithms. Their regularized limits at the point 1 (approached with unit speed from below) are

$$
\zeta^{\mathrm{sv}}(w):=\mathcal{L}_{w}\left(-\overrightarrow{1}_{1}\right)
$$

and called single-valued multiple zeta values. They generate $\mathcal{Z}^{\text {sv }}$, which is strictly contained in the algebra of all multiple zeta values. Perhaps surprisingly, the numbers $\zeta^{\text {sv }}(w)$ satisfy all motivic relations between ordinary multiple zeta values.

1.3.1. Genus 1. The universal elliptic KZB equation $[8,9,16,19,25]$ that we shall use is

$$
d J(z)=\omega J(z)
$$

where $\omega$ is the formal 1-form:

$$
\omega=-\frac{d q}{q} \operatorname{ad}\left(\varepsilon_{0}\right)+\sum_{n \geqslant 1} \frac{2}{(2 n) !} \mathbb{G}_{2 n+2}(q) \frac{d q}{q} \varepsilon_{2 n+2} .
$$

It is obtained from the restriction of the full KZB connection first to the moduli scheme of elliptic curves equipped with a nonzero tangent vector [19, equation (13.1)] and from that to the zero section of the universal elliptic curve after setting $\varepsilon_{2}$ to zero (that is, by taking its image in the Lie algebra quotient by the central derivation $\varepsilon_{2}$. This has the effect of removing a term involving $\mathbb{G}_{2}$ from the KZB connection. Since $\varepsilon_{2}$ is central, the space of equivariant modular forms we shall obtain is only marginally smaller than if we had included the term involving $\mathbb{G}_{2}$.) The coefficients $\varepsilon_{2 n}$, for all $n \geqslant 0$ are derivations of the free Lie algebra on two generators Lie(a, b). They were first written down by Tsunogai [35] in the $\ell$-adic context, and studied by Nakamura [31]. They are uniquely determined by the formulae $\varepsilon_{2 n}[a, b]=0, \varepsilon_{2 n}(\mathrm{a}) \in[\operatorname{Lie}(\mathrm{a}, \mathrm{b}), \operatorname{Lie}(\mathrm{a}, \mathrm{b})]$ and

$$
\varepsilon_{2 n}(\mathrm{~b})=-\mathrm{ad}(\mathrm{b})^{2 n} \mathrm{a}
$$


They generate (together with an $\mathfrak{s l}_{2}$-action) a Lie algebra we denote by $\mathfrak{u}^{\text {geom }}$, and satisfy many interesting quadratic relations associated with cusp forms [32] (which will be a consequence of the orthogonality condition (5) in Theorem 1.1). Let $\mathcal{U}^{\text {geom }}$ denote the affine group scheme whose Lie algebra is the completion of $\mathfrak{u}^{\text {geom }}$. It admits a right action by $\mathrm{SL}_{2}$. The function $J$ is a multivalued function of $z \in \mathfrak{H}$ taking values in $\mathcal{U}^{\text {geom }}(\mathbb{C})$. Our first theorem proves the existence of a genus one analogue of $\mathcal{L}$.

THEOREM 1.2. There exists a real-analytic function

$$
J^{\text {eqv }}: \mathfrak{H} \longrightarrow \mathcal{U}^{\text {geom }}(\mathbb{C})
$$

which satisfies the equation

$$
\frac{\partial}{\partial \tau} J^{\mathrm{eqv}}=\omega J^{\mathrm{eqv}}
$$

and is equivariant for the action of $\mathrm{SL}_{2}(\mathbb{Z})$, that is,

$$
\left.J^{\mathrm{eqv}}(\gamma \tau)\right|_{\gamma}=J^{\mathrm{eqv}}(\tau) \quad \text { for all } \gamma \in \mathrm{SL}_{2}(\mathbb{Z})
$$

The generating series $J^{\text {eqv }}$, unlike its genus zero counterpart $\mathcal{L}$, is only welldefined up to right multiplication by an element $a \in \mathcal{U}^{\text {geom }}\left(\mathcal{Z}^{\mathrm{sv}}\right)^{\mathrm{SL}_{2}}$. The series $J^{\text {eqv }}$ can be viewed as a formal power series in certain coefficients, which are maps

$$
c\left(J^{\mathrm{eqv}}\right): \mathfrak{H} \longrightarrow V_{2 n} \otimes_{\mathbb{Q}} \mathbb{C},
$$

where $V_{2 n}=\bigoplus_{r+s=2 n} X^{r} Y^{s} \mathbb{Q}$ is the space of homogeneous polynomials in $X, Y$ (corresponding to $\mathrm{b}$ and $\mathrm{a}$ ), equipped with a right action of $\mathrm{SL}_{2}$. The coefficients $c\left(J^{\text {eqv }}\right)$ are vector-valued real-analytic modular forms and satisfy

$$
c\left(J^{\mathrm{eqv}}\right)(\gamma \tau)=\left.c\left(J^{\mathrm{eqv}}\right)(\tau)\right|_{\gamma}
$$

for all $\gamma \in \mathrm{SL}_{2}(\mathbb{Z})$. Any such function can be uniquely written in the form

$$
c\left(J^{\mathrm{eqv}}\right)(\tau)=\sum_{r+s=2 n} c_{r, s}(\tau)(X-\tau Y)^{r}(X-\bar{\tau} Y)^{s},
$$

where $c_{r, s}: \mathfrak{H} \rightarrow \mathbb{C}$ are real analytic and modular of weights $(r, s)$.

Definition 1.3. The space $\mathcal{M I}^{E}$ is the $\mathcal{Z}^{\text {sv }}$-module generated by all $c\left(J^{\text {eqv }}\right)_{r, s}(\tau)$. It is independent of the choice of solution $J^{\text {eqv }}$.

One could equivalently set up the theory using vector-valued modular forms $c\left(J^{\text {eqv }}\right)$ instead of the constituent functions $c_{r, s}$. This is merely a matter of choice: we opted for the latter because of potential applications to physics. 
1.4. Further work. There are a number of questions that we have not addressed.

(1) A mixed elliptic motive is a mixed Tate motive over $\mathbb{Z}$, equipped with an action of a certain Lie algebra on, say, its de Rham realization. This Lie algebra is conjecturally isomorphic to $\mathfrak{u}^{\text {geom }}$. One can define motivic lifts of the functions $f \in \mathcal{M I}^{E}$ whose coefficients will be single-valued motivic multiple zeta values. In this precise sense, the functions in $\mathcal{M I}^{E}$ are associated with mixed Tate motives over $\mathbb{Z}$ carrying some extra structure. In particular, they will inherit an action of the motivic Galois group of mixed Tate motives over $\mathbb{Z}$, whose action on $\mathfrak{u}^{\text {geom }}$ was studied in [5], and is known to lowest orders.

(2) The functions in $\mathcal{M I}^{E}$ provide kernels for the Rankin-Selberg method. In lengths zero and one, these can be computed by the unfolding technique. It would be interesting to extend this to higher lengths.

(3) The functions defined in this paper are constructed out of the action of the real Frobenius $F_{\infty}$ on the unipotent fundamental group of the universal elliptic curve. It would be interesting to study $p$-adic versions, which should be constructed in a similar manner using the Frobenius at a finite prime.

Classical holomorphic modular forms have algebraic coefficients and admit an action of the usual Galois group. The modular forms constructed here have coefficients that are periods [26]. Remark (1) suggests that they admit an action by a motivic Galois group.

One motivation for this construction was the long-standing problem of defining a natural class of functions that contain the modular graph functions arising in genus one closed string perturbation theory [12-14, 18, 37]. This problem was briefly discussed in [6], and has an extensive literature. In this paper, we construct the class of functions which we expect to solve this problem completely. Indeed, a relation between modular graph functions and the class $\mathcal{M} \mathcal{I}^{E}\left[\mathbb{L}, \mathbb{L}^{-1}\right]$ should follow by a version of the argument given in the author's thesis. The key point is that the bar de Rham complex defined in [8] has trivial cohomology. If as expected, the modular graph functions are indeed contained in $\mathcal{M} \mathcal{I}^{E}\left[\mathbb{L}, \mathbb{L}^{-1}\right]$, then the properties of this class of functions which are proved in this paper would immediately imply most of the open conjectures and phenomena that have been experimentally observed for modular graph functions.

\section{Notations and background on the class of functions $\mathcal{M}$}

We recall some notations and background from $[6,36]$. 
2.1. General definitions. We write $z=x+i y, q=\exp (2 \pi i z)$ and set

$$
\mathbb{L}=\log |q|=\frac{1}{2} \log q \bar{q}=\pi i(z-\bar{z})=-2 \pi y .
$$

Consider the bigraded algebra of real-analytic functions [6]

$$
\mathcal{M}=\bigoplus_{r, s} \mathcal{M}_{r, s}
$$

which are modular of weights $(r, s)$ and admit an expansion of the form (1.4), where $a_{m, n}^{(k)}$ are arbitrary complex numbers. The quantity $w=r+s$ is called the total modular weight. The space $\mathcal{M}_{r, s}=0$ if $w$ is odd. The constant part of $f$ (called the Laurent polynomial or zeroth Fourier mode in the physics literature) is defined to be

$$
f^{0}=\sum_{k} a_{0,0}^{(k)} \mathbb{L}^{k} \in \mathbb{C}\left[\mathbb{L}, \mathbb{L}^{-1}\right] .
$$

The subspace of cusp forms $\mathcal{S} \subset \mathcal{M}$ is defined to be $\operatorname{ker}\left(f \mapsto f^{0}: \mathcal{M} \rightarrow \mathbb{C}[\mathbb{L}\right.$, $\left.\mathbb{L}^{-1}\right]$ ). There is a decreasing filtration by the order of poles in $\mathbb{L}$ :

$$
P^{p} \mathcal{M}=\left\{f \in \mathcal{M}: a_{m, n}^{(k)}(f)=0 \text { if } k<p\right\},
$$

which satisfies $P^{a} \mathcal{M} \times P^{b} \mathcal{M} \subset P^{a+b} \mathcal{M}$. In this paper, we shall work in the subspace

$$
\bigoplus_{r, s \geqslant 0} P^{-r-s} \mathcal{M}_{r, s} \subset \mathcal{M}
$$

with nonnegative modular weights $(r, s)$, and whose poles in $\mathbb{L}$ are bounded by $w$.

2.2. Differential operators. For all $r, s \in \mathbb{Z}$, there exist operators

$$
\partial_{r}: \mathcal{M}_{r, s} \longrightarrow \mathcal{M}_{r+1, s-1}, \quad \bar{\partial}_{s}: \mathcal{M}_{r, s} \longrightarrow \mathcal{M}_{r-1, s+1},
$$

which are variants of the Maass raising and lowering operators, defined by

$$
\partial_{r}=(z-\bar{z}) \frac{\partial}{\partial z}+r, \quad \bar{\partial}_{s}=(\bar{z}-z) \frac{\partial}{\partial \bar{z}}+s .
$$

If we extend the action of $\partial_{r}, \bar{\partial}_{s}$ to all of $\mathcal{M}$ to be the zero map on all components $\mathcal{M}_{i, j}$ for $i \neq r$ or $j \neq s$, respectively, we obtain bigraded differential operators

$$
\partial=\sum_{r} \partial_{r}: \mathcal{M} \longrightarrow \mathcal{M} \text { and } \bar{\partial}=\sum_{s} \bar{\partial}_{s}: \mathcal{M} \longrightarrow \mathcal{M}
$$


of modular weights $(1,-1)$ and $(-1,1)$, respectively. Recall that $\mathbb{L} \in \mathcal{M}_{-1,-1}$. The operators act upon it via $\partial(\mathbb{L})=\bar{\partial}(\mathbb{L})=0$, and generate a copy of $\mathfrak{s l}_{2}$ acting upon $\mathcal{M}$ :

$$
[h, \partial]=2 \partial, \quad[h, \bar{\partial}]=-2 \bar{\partial}, \quad[\partial, \bar{\partial}]=h,
$$

where $h: \mathcal{M} \rightarrow \mathcal{M}$ is multiplication by $r-s$ on the component $\mathcal{M}_{r-s}$ of $\mathcal{M}$. In [6], it is shown that the kernels of the operators $\partial, \bar{\partial}$ are the direct sums over $r, s$ of

$$
\begin{aligned}
& \operatorname{ker}\left(\partial_{r}: \mathcal{M}_{r, s} \rightarrow \mathcal{M}_{r+1, s-1}\right)=\mathbb{L}^{-r} \bar{M}_{s-r} \\
& \operatorname{ker}\left(\bar{\partial}_{s}: \mathcal{M}_{r, s} \rightarrow \mathcal{M}_{r-1, s+1}\right)=\mathbb{L}^{-s} M_{r-s},
\end{aligned}
$$

where $M_{n}$ and $\bar{M}_{n}$ denote the spaces of holomorphic and antiholomorphic modular forms, respectively. In particular, $\operatorname{ker} \partial \cap \operatorname{ker} \bar{\partial}=\mathbb{C}\left[\mathbb{L}, \mathbb{L}^{-1}\right]$.

2.3. Laplace operator. For any $r, s \in \mathbb{Z}$, the Laplace operator $\Delta_{r, s}: \mathcal{M}_{r, s} \rightarrow$ $\mathcal{M}_{r, s}$ is defined by the equivalent formulae

$$
\Delta_{r, s}=-\bar{\partial}_{s-1} \partial_{r}+r(s-1)=-\partial_{r-1} \bar{\partial}_{s}+s(r-1) .
$$

The component $\Delta_{0,0}$ is the Laplace-Beltrami operator. Let us denote by $\Delta: \mathcal{M} \rightarrow$ $\mathcal{M}$ the linear operator that acts by $\Delta_{r, s}$ on the component $\mathcal{M}_{r, s}$.

2.4. Petersson inner product. For any integer $n$, the map

$$
f \times g \mapsto\langle f, g\rangle:=\int_{\mathcal{D}} f(z) \overline{g(z)} y^{n} d \mathrm{vol}
$$

defines a pairing $\mathcal{M}_{r, s} \times \mathcal{S}_{n-s, n-r} \rightarrow \mathbb{C}$, where

$$
\mathcal{D}=\left\{|z|>1,|\operatorname{Re}(z)|<\frac{1}{2}\right\} \quad \text { and } \quad d \mathrm{vol}=\frac{d x d y}{y^{2}}
$$

are the interior of the standard fundamental domain for the action of $\mathrm{SL}_{2}(\mathbb{Z})$ on $\mathfrak{H}$, and the $\mathrm{SL}_{2}(\mathbb{Z})$-invariant volume form on $\mathfrak{H}$ in its standard normalization.

Restricting to holomorphic cusp forms $S_{n} \subset M_{n}$, we deduce pairings

$$
\langle f, g\rangle: \mathcal{M}_{r, s} \times S_{r-s} \longrightarrow \mathbb{C} \text { and }\langle f, \bar{g}\rangle: \mathcal{M}_{r, s} \times \bar{S}_{s-r} \longrightarrow \mathbb{C} .
$$

Since the Petersson inner product is nondegenerate on holomorphic cusp forms, these two pairings are equivalent to a linear map

$$
p=\left(p^{h}, p^{a}\right): \mathcal{M}_{r, s} \longrightarrow S_{r-s} \oplus \bar{S}_{s-r},
$$

whose components are called the holomorphic and antiholomorphic projections [34]. 
THEOREM 2.1. Let $f \in \mathcal{M}_{r, s}$. If $f=\partial F$ for some $F \in \mathcal{M}$, then

$$
\langle f, g\rangle=0 \quad \text { for all } g \in S_{r-s} \text { holomorphic. }
$$

In particular, $f$ is in the kernel of the holomorphic projection (2.8).

This can be written as $p^{h} \partial=0$. By taking the complex conjugate, $p^{a} \bar{\partial}=0$.

2.5. Example: real-analytic Eisenstein series. Recall the functions $\mathcal{E}_{r, s} \in$ $P^{-w} \mathcal{M}_{r, s}$ defined in (1.3). They satisfy the system of differential equations

$$
\begin{aligned}
\partial \mathcal{E}_{w, 0} & =\mathbb{L} \mathbb{G}_{w+2} \\
\partial \mathcal{E}_{r, s}-(r+1) \mathcal{E}_{r+1, s-1} & =0 \quad \text { for all } 1 \leqslant s \leqslant w
\end{aligned}
$$

and

$$
\begin{aligned}
\bar{\partial} \mathcal{E}_{0, w} & =\mathbb{L} \overline{\mathbb{G}}_{w+2} \\
\bar{\partial} \mathcal{E}_{r, s}-(s+1) \mathcal{E}_{r-1, s+1} & =0 \quad \text { for all } 1 \leqslant r \leqslant w .
\end{aligned}
$$

They are eigenfunctions of the Laplacian: $\Delta \mathcal{E}_{r, s}=-w \mathcal{E}_{r, s}$, with constant part

$$
\mathcal{E}_{r, s}^{0}=\frac{-B_{w+2}}{2(w+1)(w+2)} \mathbb{L}+\frac{(-1)^{s}}{2} \frac{w !}{2^{w}}\left(\begin{array}{l}
w \\
r
\end{array}\right) \zeta(w+1) \mathbb{L}^{-w} .
$$

This example is fairly atypical - in general, the differential equations with respect to the holomorphic and antiholomorphic differentials $\partial$ and $\bar{\partial}$ will not be symmetric. The theory is set up in such a manner as to make the differential structure with respect to $\partial$ as simple as possible, at the cost of losing explicit control over the action of $\bar{\partial}$. This is entirely analogous to the situation in genus 0 , since $\mathcal{L}$ satisfies a complicated differential equation with respect to $\frac{\partial}{\partial \bar{z}}$ whose coefficients involve multiple zeta values.

Let us write

$$
\mathcal{E}(\tau)=\sum_{r+s=2 w} \mathcal{E}_{r, s}(\tau)(X-\tau Y)^{r}(X-\bar{\tau} Y)^{s} .
$$

It is equivariant: $\mathcal{E}(\gamma \tau)=\left.\mathcal{E}(\tau)\right|_{\gamma}$ for all $\gamma \in \mathrm{SL}_{2}(\mathbb{Z})$. Consider the equivariant 1-form

$$
\underline{E}_{2 w+2}(\tau)=2 \pi i \mathbb{G}_{2 w+2}(\tau)(X-\tau Y)^{2 w} d \tau .
$$

Note that the normalization of the power of $2 \pi i$ in this expression can be different in different contexts [2]. Equations (2.10) and (2.11) are equivalent to the differential equation

$$
d \mathcal{E}=\frac{1}{2}\left(\underline{E}_{2 w+2}(\tau)+\underline{E}_{2 w+2}(\tau)\right)=\operatorname{Re}\left(\underline{E}_{2 w+2}(\tau)\right) .
$$


The functions $\mathcal{E}_{r, s}$ are thus obtained from the real part of an indefinite integral of a holomorphic Eisenstein series. This is the prototype for the general theory.

\section{Reminders on $\mathrm{SL}_{2}$-representations}

Throughout this paper, all tensors are over $\mathbb{Q}$ unless otherwise indicated.

3.1. Definitions. For all $n \geqslant 0$, define $V_{2 n}=\bigoplus_{r+s=2 n} X^{r} Y^{s} \mathbb{Q}$, equipped with the right action of $\mathrm{SL}_{2}$, and hence $\mathrm{SL}_{2}(\mathbb{Z})$, given by

$$
\left.(X, Y)\right|_{\gamma}=(a X+b Y, c X+d Y)
$$

for $\gamma$ of the form (1.1). There is an isomorphism of $\mathrm{SL}_{2}$-representations

$$
V_{2 m} \otimes V_{2 n} \cong V_{2 m+2 n} \oplus V_{2 m+2 n-2} \oplus \cdots \oplus V_{2|m-n|} .
$$

Consider the particular $\mathrm{SL}_{2}$-equivariant projector

$$
\delta^{k}: V_{2 m} \otimes V_{2 n} \longrightarrow V_{2 m+2 n-2 k}
$$

defined by

$$
\delta^{k}=\mu \circ\left(\frac{\partial}{\partial X} \otimes \frac{\partial}{\partial Y}-\frac{\partial}{\partial Y} \otimes \frac{\partial}{\partial X}\right)^{k},
$$

where $\mu: \mathbb{Q}[X, Y] \otimes \mathbb{Q}[X, Y] \rightarrow \mathbb{Q}[X, Y]$ is the multiplication map.

Recall the standard notation for generators of $\mathrm{SL}_{2}(\mathbb{Z})$ :

$$
S=\left(\begin{array}{cc}
0 & -1 \\
1 & 0
\end{array}\right), \quad T=\left(\begin{array}{ll}
1 & 1 \\
0 & 1
\end{array}\right) .
$$

3.2. de Rham version. It is convenient to define a de Rham version $V_{2 n}^{d R}$ of the (Betti) vector space $V_{2 n}$ generated by elements denoted by $\mathrm{X}$ and $\mathrm{Y}$.

There is a comparison isomorphism

$$
\begin{aligned}
V_{2 n}^{d R} \otimes \mathbb{C} & \stackrel{\sim}{\longrightarrow} V_{2 n} \otimes \mathbb{C} \\
(\mathrm{X}, \mathrm{Y}) & \mapsto\left(X,(2 \pi i)^{-1} Y\right) .
\end{aligned}
$$

The reason for this is that $X$ and $Y$ will span copies of $\mathbb{Q}(0)$ and $\mathbb{Q}(1)$, respectively: for example, $X$ is a Betti basis for $\mathbb{Q}(0)$ and $X$ is a de Rham basis.

The vector space $V_{2 n}^{d R}$ admits a (de Rham) action of $\mathrm{SL}_{2}(\mathbb{Z})$ on the right via

$$
\left.(\mathrm{X}, \mathrm{Y})\right|_{\gamma}=(a \mathrm{X}+b \mathrm{Y}, c \mathrm{X}+d \mathrm{Y})
$$


for $\gamma$ of the form (1.1). When the context is not clear, we shall denote this copy of $\mathrm{SL}_{2}$ by $\mathrm{SL}_{2}^{d R}$. The comparison isomorphism is an isomorphism of group schemes

$$
\operatorname{comp}_{B, d R}: \mathrm{SL}_{2} \times \mathbb{C} \stackrel{\sim}{\longrightarrow} \mathrm{SL}_{2}^{d R} \times \mathbb{C}
$$

which on the level of points is given by conjugation by $\left(\begin{array}{cc}1 & 0 \\ 0 & (2 \pi i)^{-1}\end{array}\right)$ :

$$
\operatorname{comp}_{B, d R}\left(\begin{array}{ll}
a & b \\
c & d
\end{array}\right)=\left(\begin{array}{cc}
a & 2 \pi i b \\
(2 \pi i)^{-1} c & d
\end{array}\right) .
$$

Consequently, the Betti action of $\mathrm{SL}_{2}(\mathbb{Z})$ on $V_{2 n}^{d R} \otimes \mathbb{C}$ is twisted by powers of $2 \pi i$. The images of the Betti elements $S$ and $T$ under comp ${ }_{B, d R}$ are

$$
S^{\prime}=\left(\begin{array}{cc}
0 & -2 \pi i \\
(2 \pi i)^{-1} & 0
\end{array}\right), \quad T^{\prime}=\left(\begin{array}{cc}
1 & 2 \pi i \\
0 & 1
\end{array}\right) .
$$

If bar denotes complex conjugation, these satisfy $\overline{S^{\prime}}=-S^{\prime}$ and $\overline{T^{\prime}}=\left(T^{\prime}\right)^{-1}$.

REMARK 3.1. There is a de Rham version of the projection $\delta_{d R}: V_{2 m}^{d R} \otimes V_{2 n}^{d R} \rightarrow$ $V_{2 n+2 m-k}$ defined in an identical manner to (3.1), except that we replace $X, Y$ with $\mathrm{X}, \mathrm{Y}$. Under the comparison isomorphism, $\delta_{d R}^{k}$ corresponds to $(2 \pi i)^{k} \delta^{k}$.

\section{Iterated integrals of Eisenstein series}

4.1. Preamble on filtrations. In order to keep the paper as accessible as possible, we work mostly with Lie algebras and iterated integrals and gloss over the geometric foundations. From time to time, a paragraph marked with a star explains the geometric background with references for the interested reader. Only in Section 7, which is considerably more technical, do we require any substantial Hodge theory and Tannakian theory of fundamental groups and their completions. As a result, the objects described in this and later sections are equipped with a limiting mixed Hodge structure and in particular, possess three filtrations: $W$, $M$ and $F$. Since all mixed Hodge structures considered here are of mixed Tate type, the monodromy-weight filtration $M$ is split in the de Rham realization by the Hodge filtration $F$, and is therefore associated with a grading, which we call the $M$-degree. It also determines $F$, which will not be discussed again. The geometric weight filtration $W$ plays a relatively minor role in this paper. Indeed, it is canonically split in the de Rham realization, and the $W$-degrees can be deduced from the $M$-degrees and $\mathfrak{s l}_{2}$-weights. It is related to the depth filtration [5], which is briefly discussed in Section 5.4. For this reason, we shall emphasize the $M$ degree, and the interested reader can look up the $W$-filtrations in $[2,21]$. 
4.2. A tensor algebra. The generators $\mathrm{X}, \mathrm{Y}$ of $V_{2 n}^{d R}$ satisfy

$$
\operatorname{deg}_{M} X=0, \quad \operatorname{deg}_{M} Y=-1
$$

Let $\mathbf{e}_{2 n+2}$ be a symbol that corresponds to the Eisenstein series of weight $2 n+2$. It is assigned an $M$-degree of -1 (it spans a copy of $\mathbb{Q}(1)$ ). Our convention is that typeface in sans serif font denotes elements in a de Rham realization.

DEFINITION 4.1. Let $\mathfrak{u}_{E}^{d R}$ denote the free graded Lie algebra generated by elements

$$
\mathbf{e}_{2 n+2} \mathrm{X}^{i} \mathrm{Y}^{2 n-i} \quad \text { for } 0 \leqslant i \leqslant 2 n
$$

and for all $n \geqslant 1$. Equivalently, it is the free graded Lie algebra generated by $\mathbf{e}_{2 n+2} V_{2 n}^{d R}$, for every $n \geqslant 1$. It is naturally equipped with a right action of $\mathrm{SL}_{2}^{d R}$.

The completed Lie algebra $\widehat{\mathfrak{u}}_{E}^{d R}$ is the Lie algebra of a pro-unipotent affine group scheme $\mathcal{U}_{E}^{d R}$ over $\mathbb{Q}$. We remark straight away that this object is not natural from a geometric perspective (it is not the de Rham realization of a motive), but will be very convenient for computational purposes. Its affine ring is the tensor coalgebra

$$
\mathcal{O}\left(\mathcal{U}_{E}^{d R}\right)=T^{c}\left(\bigoplus_{n \geqslant 1} \mathbf{E}_{2 n+2}\left(\check{V}_{2 n}^{d R}\right)\right)
$$

where $\mathbf{E}_{2 n+2}$ are symbols dual to $\mathbf{e}_{2 n+2}$, and where for any graded vector space $W$, the tensor coalgebra is defined to be the bigraded vector space

$$
T^{c}(W)=\bigoplus_{n \geqslant 0} W^{\otimes n}
$$

equipped with the shuffle product $\amalg$, and the deconcatenation coproduct

$$
\begin{aligned}
\Delta: T^{c}(W) & \longrightarrow T^{c}(W) \otimes T^{c}(W) \\
\Delta\left(w_{1} \otimes \cdots \otimes w_{n}\right) & =\sum_{0 \leqslant i \leqslant n}\left(w_{1} \otimes \cdots \otimes w_{i}\right) \otimes\left(w_{i+1} \otimes \cdots \otimes w_{n}\right) .
\end{aligned}
$$

The ring $\mathcal{O}\left(\mathcal{U}_{E}^{d R}\right)$ and the group scheme $\mathcal{U}_{E}^{d R}$ inherit a left (respectively, right) action of $\mathrm{SL}_{2}^{d R}$. Let us denote also by $\widehat{\mathcal{U}}_{E}^{d R}$ the completed universal enveloping algebra of $\mathfrak{u}_{E}^{d R}$. It is the ring of noncommutative formal power series in $\mathbf{e}_{2 n+2} \mathrm{X}^{i} \mathrm{Y}^{2 n-i}$, for $0 \leqslant i \leqslant 2 n$. We can view the points of $\mathcal{U}_{E}^{d R}$ as the set of group-like elements in $\widehat{\mathcal{U}}_{E}^{d R}$. 
4.3. Power series connection. Recall (2.13) that

$$
\begin{aligned}
\underline{E}_{2 n+2}(\tau) & =2 \pi i \mathbb{G}_{2 n+2}(\tau)(X-\tau Y)^{2 n} d \tau \\
& \stackrel{(3.3)}{=} \mathbb{G}_{2 n+2}(q)(\mathrm{X}-\log (q) \mathrm{Y})^{2 n} \frac{d q}{q} .
\end{aligned}
$$

The second expression has only rational coefficients in $q, \log (q)$.

Consider the following formal 1-form taking values in $\Omega^{1}(\mathfrak{H}) \widehat{\otimes} \widehat{\mathcal{U}}_{E}^{d R}$ :

$$
\Omega^{E}(\tau)=\sum_{n \geqslant 1} \underline{E}_{2 n+2}(\tau) \mathbf{e}_{2 n+2} .
$$

It is equivariant with respect to the (Betti) action of $\mathrm{SL}_{2}(\mathbb{Z})$ :

$$
\left.\Omega^{E}(\gamma \tau)\right|_{\gamma}=\Omega^{E}(\tau) \text { for all } \gamma \in \mathrm{SL}_{2}(\mathbb{Z}) .
$$

Here, and throughout this section, the (Betti) action of $\mathrm{SL}_{2}(\mathbb{Z})$ on the de Rham group $\mathcal{U}_{E}^{d R}(\mathbb{C})$ is via the comparison map (3.4). Consider the trivial vector bundle $\widehat{\mathcal{U}}_{E}^{d R} \widehat{\otimes} \mathbb{C}$ over $\mathfrak{H}$, equipped with the connection

$$
\nabla: \widehat{\mathcal{U}}_{E}^{d R} \widehat{\otimes} \mathbb{C} \longrightarrow \Omega^{1}(\mathfrak{H}) \widehat{\otimes} \widehat{\mathcal{U}}_{E}^{d R}
$$

defined by $\nabla=d+\Omega^{E}$, where the elements $\mathbf{e}_{2 n+2}$ act on $\widehat{\mathcal{U}}_{E}^{d R}$ by concatenation on the left. This connection is integrable since $d \Omega^{E}=\Omega^{E} \wedge \Omega^{E}=0$.

Proposition 4.2. There exists a canonical horizontal section,

$$
I^{E}: \mathfrak{H} \longrightarrow \widehat{\mathcal{U}}_{E}^{d R}(\mathbb{C})
$$

which is regularized at the unit tangent vector $\overrightarrow{1}_{\infty}$ at the cusp (equivalently, the tangent vector $\partial / \partial q$ of length one in the coordinate $q=e^{2 \pi i \tau}$ at the origin of the $q$-disk). It is given explicitly by the generating series of iterated integrals

$$
\begin{aligned}
I^{E}(\tau) & =1+\int_{\tau}^{\overrightarrow{1}_{\infty}} \Omega^{E}+\int_{\tau}^{\overrightarrow{1}_{\infty}} \Omega^{E} \Omega^{E}+\cdots \\
& =\sum_{r \geqslant 0} \sum_{n_{1}, \ldots, n_{r} \geqslant 1} \mathbf{e}_{2 n_{1}+2} \ldots \mathbf{e}_{2 n_{r}+2} \int_{\tau}^{\overrightarrow{1}_{\infty}} \underline{E}_{2 n_{1}+2}(\tau) \ldots \underline{E}_{2 n_{r}+2}(\tau),
\end{aligned}
$$

where we integrate from the left. It satisfies the following properties:

(i) $d I^{E}(\tau)=-\Omega^{E}(\tau) I^{E}(\tau)$, 


$$
\begin{aligned}
& \text { (ii) } I^{E}\left(\overrightarrow{1}_{\infty}\right)=1, \\
& \text { (iii) } I^{E}(\tau) \in \mathcal{U}_{E}^{d R}(\mathbb{C}) \quad \text { for all } \tau \in \mathfrak{H} .
\end{aligned}
$$

Furthermore, the coefficient of $\mathbf{e}_{2 n_{1}+2} \ldots \mathbf{e}_{2 n_{r}+2} \mathrm{X}^{i_{1}} \mathrm{Y}^{2 n_{1}-i_{1}} \otimes \cdots \otimes \mathrm{X}^{i_{r}} \mathrm{Y}^{2 n_{r}-i_{r}}$ in $I^{E}(\tau)$ is an element of the ring $\mathbb{C}[[q]][\log (q)]$, and more precisely is a polynomial in $\log (q)$ of degree at most $2\left(n_{1}+\cdots+n_{r}\right)+r$ whose coefficients are convergent power series in $q$.

Proof. The definition of the tangential base point $\overrightarrow{1}_{\infty}$ is given in [2, Section 2], as well as a concrete explanation of the regularized iterated integrals. Parts (i), (ii) and (iii) are proved in [2, Propositions 1 and 2]. Only the last part remains to be proven. For this, observe that the space of multivalued functions in the $q$-disk admitting a convergent expansion in the ring $\mathbb{C}[[q]][\log (q)]$ is closed under $f \mapsto$ $\int f q^{-1} d q$, that is, if $f \in \mathbb{C}[[q]][\log (q)]$, then there exists $F \in \mathbb{C}[[q]][\log (q)]$ such that $d F=q^{-1} f d q$. The series $F$ converges, and defines a multivalued holomorphic function on the $q$-disk. Furthermore, a primitive of $q^{i} \log ^{j}(q) d q$ is again a polynomial in $q$ and $\log q$ of degree at most $j+1$ in $\log (q)$. Since the coefficients of $\underline{E}_{2 n+2}(\tau)$ lie in $\mathbb{C}[[q]][\log (q)] d q$ and have degree at most $2 n$ in $\log q$, the statement follows.

Properties (i) and (ii) in the previous proposition determine $I^{E}(\tau)$ uniquely. Property (iii) is equivalent to a shuffle product relation between iterated integrals of Eisenstein series, which is spelt out in [2, (3.8)].

COROLlaRY 4.3. For every $\gamma \in \mathrm{SL}_{2}(\mathbb{Z})$, there is a unique $\mathcal{C}_{\gamma}^{E} \in \mathcal{U}_{E}^{d R}(\mathbb{C})$ such that

$$
\left.I^{E}(\gamma \tau)\right|_{\gamma} \mathcal{C}_{\gamma}^{E}=I^{E}(\tau)
$$

for all $\tau \in \mathfrak{H}$. It satisfies the cocycle equation

$$
\mathcal{C}_{g h}^{E}=\left.\mathcal{C}_{g}^{E}\right|_{h} \mathcal{C}_{h}^{E} \quad \text { for all } g, h \in \mathrm{SL}_{2}(\mathbb{Z})
$$

Proof. This is identical to [2, Lemma 5.1], bearing in mind that the right action of $\mathrm{SL}_{2}(\mathbb{Z})$ on $\mathcal{U}_{E}^{d R}(\mathbb{C})$ is given by (3.4).

The map $\gamma \mapsto \mathcal{C}_{\gamma}^{E}$ defines a non-Abelian cocycle

$$
\mathcal{C}^{E} \in Z^{1}\left(\mathrm{SL}_{2}(\mathbb{Z}), \mathcal{U}_{E}^{d R}(\mathbb{C})\right)
$$

Since $\mathrm{SL}_{2}(\mathbb{Z})$ is finitely presented, and generated by the two elements $T, S$, any cocycle $\mathcal{C}$ is uniquely determined [2, Lemma 5.5] by its values $\mathcal{C}_{S}$ and $\mathcal{C}_{T}$, which 
satisfy

$$
\begin{aligned}
\left.\mathcal{C}_{S}\right|_{S} \mathcal{C}_{S} & =1 \\
\left.\left.\mathcal{C}_{U}\right|_{U^{2}} \mathcal{C}_{U}\right|_{U} \mathcal{C}_{U} & =1,
\end{aligned}
$$

where $U=T S$ and $\mathcal{C}_{U}=\mathcal{C}_{T} \mid{ }_{S} \mathcal{C}_{S}$. The series $\mathcal{C}_{T}^{E}$ was computed explicitly in [2, Section 6], and its coefficients involve only powers of $2 \pi i$. The coefficients of $\mathcal{C}_{S}^{E}$ are numbers which we called multiple modular values in [2].

4.3.1. Geometric background*. The affine group scheme $\mathcal{U}_{E}^{d R}$ is not a good object in the sense that it does not admit a natural mixed Hodge structure (see [22, Remark 19.5]). A closely related object is the de Rham relative completion of the fundamental group of $\mathfrak{M}_{1,1}$ with respect to the unit tangent vector at the cusp $[2,23]$. The group scheme $\mathcal{U}_{E}^{d R}$ is a quotient of it (but not in the category of mixed Hodge structures). The reason for working with $\mathcal{U}_{E}^{d R}$ is for simplicity of exposition, since under the monodromy homomorphism, discussed below, all generators of relative completion that correspond to cusp forms act trivially, leaving only the Eisenstein series. These are artificially captured by $\mathcal{U}_{E}^{d R}$.

4.4. Totally holomorphic iterated integrals. For the meaning of 'totally holomorphic', see [2, Section 13.7]. In this section, we work over the ring of complex numbers, and all tensors are over $\mathbb{C}$. Consider the tensor coalgebra

$$
T^{c}\left(\bigoplus_{n \geqslant 1} M_{2 n+2}(\mathbb{C}) \otimes \check{V}_{2 n}\right),
$$

where $M_{2 n+2}(\mathbb{C})$ denotes the complex vector space of holomorphic modular forms of weight $2 n+2$. Elements in this coalgebra define 'totally holomorphic' iterated integrals on $\mathfrak{H}$. They are linear combinations of the following iterated integrals

$$
\int_{\tau}^{\tau_{0}} \omega_{1} \ldots \omega_{r}
$$

where $\omega_{k}$ are of the form $(2 \pi i) \tau^{m_{k}} f_{k}(\tau) d \tau$ with $f_{k} \in M_{2 n_{k}+2}(\mathbb{C})$ and $0 \leqslant$ $m_{k} \leqslant 2 n_{k}$. For $\tau_{0}$ a finite base point, these were considered by Manin [28, 29]. Expression (4.7) is the coefficient of $\pm X^{2 n_{1}-m_{1}} Y^{m_{1}} \otimes \cdots \otimes X^{2 n_{r}-m_{r}} Y^{m_{r}}$ in

$$
\int_{\tau}^{\tau_{0}} \underline{f_{1}}(\tau) \ldots \underline{f_{r}}(\tau)
$$

where for any modular form $f \in M_{2 n+2}(\mathbb{C})$ of weight $2 n+2$, we write

$$
\underline{f}(\tau)=2 \pi i f(\tau)(X-\tau Y)^{2 n} d \tau \text {. }
$$


A different normalization of the power of $2 \pi i$ was used in the first chapter of [2]. From the definition of iterated integrals, one has

$$
\frac{d}{d \tau} \int_{\tau}^{\tau_{0}} \omega_{1} \ldots \omega_{r}=-\omega_{1} \int_{\tau}^{\tau_{0}} \omega_{2} \ldots \omega_{r}
$$

The corresponding integrals regularized with respect to a tangent vector at the cusp were studied in [2, Section 4].

PROPOSITION 4.4. Let $\tau_{0}$ be any (possibly tangential) base point on $\mathfrak{H}$. The iterated integration homomorphism (with respect to the shuffle product on the tensor coalgebra)

$$
\int_{\tau}^{\tau_{0}}: T^{c}\left(\bigoplus_{n \geqslant 1} M_{2 n+2}(\mathbb{C}) \otimes V_{2 n}^{\vee}\right) \longrightarrow \text { holomorphic functions of } \tau \in \mathfrak{H}
$$

is injective. It follows that iterated integrals are linearly independent over the ring $M[\tau]$ generated by holomorphic modular forms and the function $\tau$.

We give two different proofs of linear independence. The first is conceptual and uses properties of relative completion. It is a generalization to vectorvalued iterated integrals of a well-known theorem on the linear independence of iterated integrals due to Chen [10]. The second proof is elementary but more computational. (Since writing this paper, a third approach appeared in [30], which treats the case of quasimodular forms for $\mathrm{SL}_{2}(\mathbb{Z})$.)

We first deduce the second part of the proposition from the linear independence.

Proof. Since the space $M$ of holomorphic modular forms is an algebra, we can multiply any linear relation amongst iterated integrals with coefficients in the ring $M[\tau]$ of polynomials in $M$ and $\tau$ by a nonzero modular form of sufficiently large weight to obtain a relation of the form

$$
\sum_{I=\left(i_{1}, \ldots, i_{n}\right)} \lambda_{I} \omega_{i_{1}} \int_{\tau}^{\tau_{0}} \omega_{i_{2}} \ldots \omega_{i_{n}}=0
$$

where $\lambda_{I} \in \mathbb{C}$ and every $\omega_{i}$ is of the form $\tau^{k} f(\tau)$ where $k+2$ is bounded above by the weight of $f$. This in turn implies, by integrating, a linear relation of the form

$$
\sum_{I=\left(i_{1}, \ldots, i_{n}\right)} \lambda_{I} \int_{\tau}^{\tau_{0}} \omega_{i_{1}} \omega_{i_{2}} \ldots \omega_{i_{n}}=\lambda
$$

where $\lambda \in \mathbb{C}$. Since the right-hand side is a multiple of the empty iterated integral 1 , this is a relation between iterated integrals. Using the fact that they are linearly independent, we deduce that all coefficients $\lambda$ vanish. 
The following corollary follows immediately from the proposition by applying it term by term in $\bar{q}, \bar{\tau}$.

COROLLARY 4.5. Let $\mathcal{I} \subset \mathbb{C}[[q]][\tau]$ denote the $\mathbb{C}$-vector space generated by the iterated integrals (4.7). Then the following natural map is an injection:

$$
\mathcal{I} \otimes M[\tau] \otimes \overline{\mathcal{I}} \otimes \bar{M}[\bar{\tau}] \longrightarrow \mathbb{C}[[q, \bar{q}]][\tau, \bar{\tau}] .
$$

4.4.1. General proof of linear independence using relative completion. Suppose that $\omega \in T^{c}\left(\bigoplus_{n \geqslant 1} M_{2 n+2}(\mathbb{C}) \otimes V_{2 n}^{\vee}\right)$ such that

$$
\int_{\tau}^{\tau_{0}} \omega=0
$$

for all $\tau \in \mathfrak{H}$. In particular, for any $\gamma \in \mathrm{SL}_{2}(\mathbb{Z})$, the integral from $\tau=\gamma^{-1} \tau_{0}$ to $\tau_{0}$ also vanishes. It follows that $\omega$ vanishes on $\pi_{1}\left(\mathfrak{M}_{1,1}(\mathbb{C}), x_{0}\right) \cong \mathrm{SL}_{2}(\mathbb{Z})$, where $\mathfrak{M}_{1,1}(\mathbb{C})$ is the orbifold quotient of $\mathfrak{H}$ by the action of $\mathrm{SL}_{2}(\mathbb{Z})$, and $x_{0}$ is the image of $\tau_{0}$. But

$$
T^{c}\left(\bigoplus_{n \geqslant 1} M_{2 n+2} \otimes V_{2 n}^{\vee}\right) \subset \mathcal{O}\left(\pi_{1}^{\mathrm{rel}, d R}\left(\mathfrak{M}_{1,1}(\mathbb{C}), x_{0}\right)\right),
$$

where $\pi_{1}^{\text {rel }}$ denotes the de Rham version of relative completion [2, 22, 23]. The topological fundamental group $\pi_{1}^{\text {top }}\left(\mathfrak{M}_{1,1}(\mathbb{C}), x_{0}\right)$ is Zariski-dense in its grouptheoretic relative completion, which is the Betti realization of the de Rham relative completion, and isomorphic to it over $\mathbb{C}$ via the comparison isomorphism. Thus $\mathrm{SL}_{2}(\mathbb{Z})$ is Zariski-dense in the latter, and we have $\omega=0$, that is, iterated integrals are linearly independent. Note that the proof works more generally for the whole of the affine ring of de Rham relative completion, and not just for totally holomorphic iterated integrals.

4.4.2. An elementary proof of linear independence. For the benefit of the reader not familiar with relative completion, we spell out the above proof of linear independence of iterated integrals in elementary terms. It only uses the EichlerShimura theorem.

Fix $\tau_{0} \in \mathfrak{H}$. Given a modular form $f$ of weight $w$ and $0 \leqslant i \leqslant w-2$, the differential one-form $\tau^{i} f(\tau) d \tau$ on $\mathfrak{H}$ defines a function

$$
\gamma \mapsto \int_{\gamma^{-1} \tau_{0}}^{\tau_{0}} \tau^{i} f(\tau) d \tau: \mathrm{SL}_{2}(\mathbb{Z}) \longrightarrow \mathbb{C},
$$

where $\gamma$ denotes the geodesic path from $\gamma^{-1} \tau_{0}$ to $\tau_{0}$. 
LEMMA 4.6. Let $f_{1}, \ldots, f_{n}$ be linearly independent holomorphic modular forms of weights $w_{1}, \ldots, w_{n}$. Then the functions $\tau^{i} f_{j}(\tau) d \tau: \mathrm{SL}_{2}(\mathbb{Z}) \rightarrow \mathbb{C}$, where $1 \leqslant$ $j \leqslant n$ and $0 \leqslant i \leqslant w_{j}-2$, are linearly independent over $\mathbb{C}$.

Proof. An element $g \in \mathrm{SL}_{2}(\mathbb{Z})$ defines an automorphism of $\mathfrak{H}$. By functoriality of integration with respect to automorphisms (change of variables formula), a linear combination of 1-forms of the above type $\omega$ vanishes on all $\gamma \in \mathrm{SL}_{2}(\mathbb{Z})$ if and only if the same is true of $g^{*} \omega$. Now observe by modularity of $f$ that

$$
\begin{aligned}
S^{*}(f(\tau) d \tau) & =\tau^{w-2} f(\tau) d \tau \\
T^{*}\left(\tau^{i} f(\tau) d \tau\right) & =(\tau+1)^{i} f(\tau) d \tau,
\end{aligned}
$$

where $w$ is the weight of $f$, and $T \tau=\tau+1, S \tau=-\tau^{-1}$. It follows that

$$
\left(T^{*}-\mathrm{id}\right)\left(\tau^{i} f(\tau) d \tau\right)=i \tau^{i-1} f(\tau) d \tau+\text { terms of lower order in } \tau^{j} f(\tau) .
$$

Equivalently, the complex vector space spanned by the $\tau^{i} f(\tau) d \tau$ for $0 \leqslant i \leqslant$ $w-2$ is an irreducible $\mathrm{SL}_{2}(\mathbb{Z})$-representation, and any nonzero vector in it generates the entire space under the action of $S$ and $T$. The following arguments are easily translated into representation-theoretic language, but we give a long-winded account for explicitness. Suppose that there exists an $\omega=$ $\sum \lambda_{i j} \tau^{i} f_{j}(\tau) d \tau$ that vanishes on $\mathrm{SL}_{2}(\mathbb{Z})$ for some $\lambda_{i j} \in \mathbb{C}$ not all zero. By applying $\left(T^{*}-\mathrm{id}\right)^{N}$ for a sufficiently large $N$, we can assume that all powers of $\tau$ are zero, that is, $\lambda_{i j}=0$ for $i>0$. By applying $S^{*}$, and once again $\left(T^{*}-\mathrm{id}\right)^{N^{\prime}}$ for some $N^{\prime}$, we can assume in addition that all $f_{j}$ have the same weight $w$. Thus we can write $\omega=\sum \lambda_{j} f_{j}(\tau) d \tau$, where $\lambda_{j} \in \mathbb{C}$ are not all zero. Via the action of $g^{*}$ for $g \in \mathrm{SL}_{2}(\mathbb{Z})$, we deduce that $\tau^{i} \omega$ vanishes as a function on $\mathrm{SL}_{2}(\mathbb{Z})$ for all $0 \leqslant i \leqslant w-2$. The same is therefore true of the formal linear combination

$$
\sum_{j} \lambda_{j} f_{j}(\tau)(X-\tau Y)^{w-2} d \tau
$$

which has coefficients in $V_{2 n}$. But this is equivalent to the statement

$$
\sum_{j} \lambda_{j} C_{f_{j}}=0
$$

where for $f \in M_{2 n+2}(\mathbb{C})$ holomorphic, $C_{f} \in Z^{1}\left(\mathrm{SL}_{2}(\mathbb{Z}), V_{2 n} \otimes \mathbb{C}\right)$ is the cocycle

$$
C_{f}=\gamma \mapsto \int_{\gamma^{-1} \tau_{0}}^{\tau_{0}} f(\tau)(X-\tau Y)^{w-2}
$$

associated with $f$. But the Eichler-Shimura theorem [15, 27, 33] implies that the map

$$
f \mapsto\left[C_{f}\right]: M_{2 n+2}(\mathbb{C}) \longrightarrow H^{1}\left(\mathrm{SL}_{2}(\mathbb{Z}), V_{2 n} \otimes \mathbb{C}\right)
$$


is injective, so a fortiori the cocycles $C_{f_{j}}$ are independent. Hence $\lambda_{j}=0$ for all $j$, a contradiction.

The following lemma is an easy exercise, but it is included for completeness.

LEMMA 4.7. Given any linearly independent functions $\phi_{1}, \ldots, \phi_{m}: S \rightarrow \mathbb{C}$, where $S$ is a set, there exist $x_{1}, \ldots, x_{m} \in \bigoplus_{s \in S} \mathbb{C} s$ such that $\phi_{i}\left(x_{j}\right)=\delta_{i j}$ for all $1 \leqslant i, j \leqslant m$, where the functions $\phi_{i}$ are extended linearly to linear combinations in $S$.

Proof. Consider the matrix-valued function $P=\left(\phi_{j}\left(s_{i}\right)\right)_{i, j}$ on $S^{m}$. Its determinant is not identically zero on $S^{m}$. For otherwise, a row expansion would yield a relation $\sum_{i=1}^{m} \lambda_{i} \phi_{i}\left(s_{m}\right)=0$, for all $s_{m}$, where the $\lambda_{i}$ are minors of $P$. By linear independence, the $\lambda_{i}$ vanish. The same argument can then be applied to each minor of $P$, and we eventually deduce by induction on the size of $P$ that every entry $\phi_{j}\left(s_{i}\right)$ vanishes for all $s_{i}$, a contradiction. Let $s_{1}, \ldots, s_{m} \in S$ such that $\operatorname{det}(P) \neq 0$. Let $e_{k}$ be the column vector with a 1 in the $k$ th row and zeros elsewhere. Let $\lambda_{k}$, for $1 \leqslant k \leqslant m$, denote the vector in $\mathbb{C}^{m}$ such that $\lambda_{k}^{T} P=e_{k}$. Set $x_{k}=\left(s_{1}, \ldots, s_{m}\right) \cdot \lambda_{k} \in \bigoplus_{s \in S} \mathbb{C} s$. By construction, it satisfies $\phi_{i}\left(x_{j}\right)=\delta_{i j}$.

Suppose that we have a nontrivial relation of minimal length $n$ for all $\tau \in \mathfrak{H}$ :

$$
\sum_{I} \lambda_{I} \int_{\tau}^{\tau_{0}} \omega_{i_{1}} \ldots \omega_{i_{n}}+(\text { iterated integrals of length } \leqslant n-1)=0,
$$

where the $\omega_{i_{j}} \in\left\{\omega_{1}, \ldots, \omega_{N}\right\}$, a set of linearly independent 1-forms of the kind $\tau^{i} f(\tau) d \tau$ where $0 \leqslant i \leqslant w$, where $f \in M_{w+2}(\mathbb{C})$. Composition of paths for iterated integrals $[10]$ states that

$$
\int_{\alpha \beta} \omega_{i_{1}} \ldots \omega_{i_{n}}=\sum_{k=0}^{n} \int_{\alpha} \omega_{i_{1}} \ldots \omega_{i_{k}} \int_{\beta} \omega_{i_{k+1}} \ldots \omega_{i_{n}},
$$

where $\alpha, \beta$ are two composable paths, and $\alpha \beta$ denotes the path $\alpha$ followed by $\beta$. By linearity, it holds more generally for $\alpha$ any linear combination of paths that can be composed with $\beta$. By the previous two lemmas, there exist elements $x_{i} \in \mathbb{C}\left[\mathrm{SL}_{2}(\mathbb{Z})\right]$ for $1 \leqslant i \leqslant N$, where $\mathrm{SL}_{2}(\mathbb{Z})$ are classes of paths based at $\tau$, such that

$$
\int_{x_{j}} \omega_{i}=\delta_{i j}
$$

Apply the composition of paths formula with $\beta$ a path from $\tau$ to $\tau_{0}$, and $\alpha=$ $x_{j}$. Since $\alpha \beta$ is a linear combination of paths from points in the $\mathrm{SL}_{2}(\mathbb{Z})$-orbit 
of $\tau$ to $\tau_{0}$ and since the above linear combination of iterated integrals vanishes along each such path, we deduce $N$ relations, for each $1 \leqslant i \leqslant N$, of the form

$$
\sum_{I} \lambda_{I} \delta_{i_{1} j} \int_{\tau}^{\tau_{0}} \omega_{i_{2}} \ldots \omega_{i_{n}}+(\text { iterated integrals of length } \leqslant n-2)=0,
$$

which have length $\leqslant n-1$. By minimality, the coefficients $\lambda_{I}$ such that $i_{1}=j$ vanish. Since this holds for all $j=1, \ldots, N$, we conclude that the $\lambda_{I}$ all vanish, a contradiction.

\section{A Lie algebra of geometric derivations}

5.1. Geometric context*. The de Rham fundamental group $\pi_{1}^{d R}\left(\mathcal{E}_{\partial / \partial q}^{\times}, \overrightarrow{1}_{0}\right)$ of the punctured first-order smoothing of the Tate curve [21, 23], with the base point given by a (choice of) unit tangent vector at the origin, is the de Rham realization of a mixed Tate motive over $\mathbb{Z}$ [20]. This last fact is not strictly required for this paper and could be circumvented at the cost of complicating some arguments. The affine ring of the de Rham realisation is canonically weight-graded, and one has an isomorphism

$$
\Pi \cong \pi_{1}^{d R}\left(\mathcal{E}_{\partial / \partial q}^{\times}, \overrightarrow{1}_{0}\right),
$$

where $\Pi$ is the pro-unipotent affine group scheme whose Lie algebra is the completion of the bigraded Lie algebra $\operatorname{Lie}(a, b)$ discussed below.

5.2. Derivations. Consider the free bigraded Lie algebra Lie( $(a, b)$ on two generators a, b. We shall only be concerned with its $M$-grading, which satisfies

$$
\operatorname{deg}_{M}(\mathrm{a})=-1, \quad \operatorname{deg}_{M}(\mathrm{~b})=0 .
$$

Recall that this is one half of the $M$-grading in [2, 21].

Let $\operatorname{Der}^{\Theta} \operatorname{Lie}(a, b)$ denote the Lie algebra of derivations

$$
\delta: \operatorname{Lie}(a, b) \longrightarrow \operatorname{Lie}(a, b),
$$

which annihilate the element $\Theta=[a, b] \in \operatorname{Lie}(a, b)$, that is,

$$
[\delta(\mathrm{a}), \mathrm{b}]+[\mathrm{a}, \delta(\mathrm{b})]=0 .
$$

Proposition-Definition 5.1. There exists a distinguished family of derivations $[21,31,32,35]$ for every $n \geqslant 0$ :

$$
\varepsilon_{2 n+2}^{\vee} \in \operatorname{Der}^{\Theta} \operatorname{Lie}(\mathrm{a}, \mathrm{b})
$$


which are uniquely determined by the property $\varepsilon_{2 n+2}^{\vee} \Theta=0$, the formula

$$
\varepsilon_{2 n+2}^{\vee}(\mathrm{a})=\operatorname{ad}(\mathrm{a})^{2 n+2}(\mathrm{~b}),
$$

and the fact that $\varepsilon_{2 n+2}^{\vee}(b)$ is of degree $\geqslant 1$ in $b$. Their action on $b$ is given by

$$
\varepsilon_{2 n+2}^{\vee}(\mathrm{b})=\frac{1}{2} \sum_{i+j=2 n+1}(-1)^{i}\left[\operatorname{ad}(\mathrm{a})^{i} \mathrm{~b}, \operatorname{ad}(\mathrm{a})^{j} \mathrm{~b}\right],
$$

where $i, j \geqslant 0$. These derivations were first written down by Tsunogai [35].

The Lie algebra Lie(a, b) admits a right action by $\mathrm{SL}_{2}^{d R}$ :

$$
\begin{aligned}
\operatorname{Lie}(\mathrm{a}, \mathrm{b}) \times \operatorname{SL}_{2}^{d R}(\mathbb{Z}) & \longrightarrow \operatorname{Lie}(\mathrm{a}, \mathrm{b}) \\
\left.(\mathrm{a}, \mathrm{b})\right|_{\gamma} & =(d \mathrm{a}+c \mathrm{~b}, b \mathrm{a}+a \mathrm{~b}),
\end{aligned}
$$

where $\gamma$ is given by (1.1). The infinitesimal version of this action gives rise to an action of the Lie algebra $\mathfrak{s l}_{2}$ via the following derivations:

$$
\varepsilon_{0}=-\mathrm{a} \frac{\partial}{\partial \mathrm{b}}, \quad \varepsilon_{0}^{\vee}=\mathrm{b} \frac{\partial}{\partial \mathrm{a}} .
$$

The notation is consistent with (5.2) since $\varepsilon_{0}^{\vee}$ is indeed the case $n=-1$ of (5.2). Since they annihilate $\Theta$, they generate a copy of $\mathfrak{s l}_{2}$ inside $\operatorname{Der}^{\Theta} \operatorname{Lie}(a, b)$ via

$$
\left[\varepsilon_{0}, \varepsilon_{0}^{\vee}\right]=\mathrm{h}, \quad\left[\mathrm{h}, \varepsilon_{0}\right]=-2 \varepsilon_{0}, \quad\left[\mathrm{~h}, \varepsilon_{0}^{\vee}\right]=2 \varepsilon_{0}^{\vee},
$$

where $h \in \operatorname{Der}^{\Theta} \operatorname{Lie}(a, b)$ is multiplication by $\operatorname{deg}_{b}-\operatorname{deg}_{a}$. The derivation algebra $\operatorname{Der}{ }^{\Theta} \operatorname{Lie}(a, b)$ therefore admits an inner action of $\mathfrak{s l}_{2}$. With our conventions, a derivation $x \in \operatorname{Der}^{\Theta} \operatorname{Lie}(\mathrm{a}, \mathrm{b})$ is a lowest weight vector if and only if it satisfies $\left[\varepsilon_{0}, x\right]=0$. It is a highest weight vector if and only if $\left[\varepsilon_{0}^{\vee}, x\right]=0$. Alternatively, one can simply assign an $\mathfrak{s l}_{2}$-weight of 1 to $b$ and -1 to $a$.

LEMMA 5.2. The elements $\varepsilon_{2 n+2}^{\vee}$ are highest weight vectors and generate an irreducible representation of $\mathfrak{s l}_{2}$ of dimension $2 n+1$, with the basis $\operatorname{ad}^{i}\left(\varepsilon_{0}^{\vee}\right) \varepsilon_{2 n+2}^{\vee}$ for $0 \leqslant i \leqslant 2 n$. In particular, $\operatorname{ad}^{2 n+1}\left(\varepsilon_{0}^{\vee}\right)\left(\varepsilon_{2 n+2}^{\vee}\right)=0$.

Proof. These facts are well known and easily verified from the definitions.

Let us denote by $\varepsilon_{2 n+2}$ the operators obtained from $\varepsilon_{2 n+2}^{\vee}$ by conjugating by the element $S$, where $\left.(\mathrm{a}, \mathrm{b})\right|_{S}=(-\mathrm{b}, \mathrm{a})$. These are lowest weight vectors and satisfy

$$
\varepsilon_{2 n+2}(\mathrm{~b})=-\operatorname{ad}(\mathrm{b})^{2 n+2}(\mathrm{a}) .
$$




\section{3. (de Rham) Lie algebra of geometric derivations}

Definition 5.3. Define a Lie subalgebra

$$
\mathfrak{u}^{\text {geom }} \leqslant \operatorname{Der}^{\Theta} \operatorname{Lie}(a, b)
$$

to be the bigraded Lie subalgebra generated by the $\varepsilon_{2 n+2}^{\vee}$ for $n \geqslant 1$, under the action of $\mathfrak{s l}_{2}$. By the previous lemma, it is the bigraded Lie subalgebra generated by the derivations $\operatorname{ad}\left(\varepsilon_{0}^{\vee}\right)^{i} \varepsilon_{2 n+2}^{\vee}$ for all $n \geqslant 1$ and $0 \leqslant i \leqslant 2 n$.

With this definition, which differs slightly from the version given in [5], $\mathfrak{u}^{\text {geom }}$ does not contain the element $\varepsilon_{2}^{\vee}=\varepsilon_{2}$, which plays a limited role, and commutes (as one may verify) with all elements of $\mathfrak{u}^{\text {geom }}$. Therefore the Lie algebra generated by $\varepsilon_{0}$ and all derivations $\varepsilon_{2 n+2}^{\vee}$ for $n \geqslant-1$ is isomorphic to

$$
\varepsilon_{2} \mathbb{Q} \oplus\left(\mathfrak{s l}_{2} \ltimes \mathfrak{u}^{\text {geom }}\right) .
$$

DEFINITION 5.4. Let $\mathcal{U}^{\text {geom }}$ denote the (de Rham) pro-unipotent affine group scheme whose Lie algebra is the completion of $\mathfrak{u}^{\text {geom }}$. It admits a right action of $\mathrm{SL}_{2}^{d R}(\mathbb{Z})$.

The complex points $\mathcal{U}^{\text {geom }}(\mathbb{C})$ can be described relatively explicitly as follows. They consist of the group-like elements in the completed universal enveloping algebra of $\mathfrak{u}^{\text {geom }}$ tensored with $\mathbb{C}$. These can be represented as certain formal power series

$$
\sum_{r \geqslant 0} \sum_{0 \leqslant m_{i} \leqslant n_{i}} \sum_{n_{1}, \ldots, n_{r} \geqslant 1} a_{n_{1}, \ldots, n_{r}}^{m_{1}, \ldots, m_{r}} \varepsilon_{2 n_{1}+2}^{\left(m_{1}\right)} \varepsilon_{2 n_{2}+2}^{\left(m_{2}\right)} \ldots \varepsilon_{2 n_{r}+2}^{\left(m_{r}\right)}
$$

with $a_{n_{1}, \ldots, n_{r}}^{m_{1}, \ldots, m_{r}} \in \mathbb{C}$, where $\varepsilon_{2 n+2}^{(m)}$ corresponds to a generator ad $\mathrm{ad}^{m}\left(-\varepsilon_{0}\right) \varepsilon_{2 n+2}$ of $\mathfrak{u}^{\text {geom }}$. Note that there are relations in $\mathfrak{u}^{\text {geom }}$, so this representation is not unique.

5.4. Relations. The elements $\varepsilon_{2 n+2}$ satisfy many nontrivial relations, which were first studied by Pollack [32] following a suggestion of Hain (in Pollack's notation, the notations $\varepsilon$ and $\varepsilon^{\vee}$ are reversed). One shows that the elements

$$
\left[\varepsilon_{0}^{i} \varepsilon_{2 a+2}, \varepsilon_{0}^{j} \varepsilon_{2 b+2}\right] \quad \text { where } 0 \leqslant i \leqslant 2 a, 0 \leqslant j \leqslant 2 b
$$

are linearly independent when $a, b \geqslant 1$ and $a+b \leqslant 4$, but starting from $M$-degree $\leqslant-12$, Pollack showed that there are relations

$$
\left[\varepsilon_{10}^{\vee}, \varepsilon_{4}^{\vee}\right]-3\left[\varepsilon_{8}^{\vee}, \varepsilon_{6}^{\vee}\right]=0
$$




$$
2\left[\varepsilon_{14}^{\vee}, \varepsilon_{4}^{\vee}\right]-7\left[\varepsilon_{12}^{\vee}, \varepsilon_{6}^{\vee}\right]+11\left[\varepsilon_{10}^{\vee}, \varepsilon_{8}^{\vee}\right]=0,
$$

which are the first two in an infinite sequence of quadratic and higher order relations. There are several different proofs of these relations in the literature. We shall derive a new and elementary interpretation of these relations via the orthogonality of equivariant iterated integrals of Eisenstein series to cusp forms (Theorem 12.3).

5.5. Embeddings. One way to think of the Lie algebra $\mathfrak{u}^{\text {geom }}$ is as a quotient of a certain free Lie algebra generated by Eisenstein symbols (see the next section). Another way is to embed it inside the free Lie algebra Lie (a, b).

Let us write $H=\mathbb{Q} \mathbf{a} \oplus \mathbb{Q} \mathrm{b}$. The natural map

$$
\operatorname{Der} \operatorname{Lie}(\mathrm{a}, \mathrm{b}) \longrightarrow \operatorname{Hom}(H, \operatorname{Lie}(\mathrm{a}, \mathrm{b}))
$$

is injective, since a derivation $\delta$ is uniquely determined by $\delta(\mathrm{a}), \delta(\mathrm{b})$. Hence

$$
\mathfrak{u}^{\text {geom }} \longrightarrow H^{\vee} \otimes_{\mathbb{Q}} \operatorname{Lie}(\mathrm{a}, \mathrm{b})
$$

is also injective. In fact, more is true. It is straightforward to show that, as a consequence of the relation $\delta(\Theta)=0$, the action of a derivation on either $a$ or $b$ defines a pair of injective linear maps

$$
e v_{x}: \delta \mapsto \delta(x): \mathfrak{u}^{\text {geom }} \longrightarrow \operatorname{Lie}(\mathrm{a}, \mathrm{b}),
$$

where $x=\mathrm{a}$ or $\mathrm{b}$. These maps do not respect the Lie algebra structure but can be used to write down elements in $\mathcal{O}\left(\mathcal{U}^{\text {geom }}\right)$. We shall only use them in Section 12.4.

\section{Monodromy homomorphism}

6.1. Geometric preamble*. Let $\mathcal{G}_{1,1}^{d R}$ denote the de Rham relative completion $[2,22]$ of the fundamental group of the moduli scheme of elliptic curves equipped with a nonzero Abelian differential, with base point the unit tangent vector at the cusp. The monodromy action defines a canonical homomorphism

$$
\mathcal{G}_{1, \overrightarrow{1}}^{d R} \longrightarrow \operatorname{Aut}\left(\pi_{1}^{d R}\left(\mathcal{E}_{\partial / \partial q}^{\times}, \overrightarrow{1}_{0}\right)\right) .
$$

After choosing suitable splittings of the weight filtrations, it gives rise to a morphism from the graded Lie algebra of $\mathcal{G}_{1,1}^{d R}$ to $\varepsilon_{2} \mathbb{Q} \oplus \mathfrak{u}^{\text {geom }}\left(\varepsilon_{2}\right.$ is central). One deduces the existence of a homomorphism

$$
\mathcal{G}_{1,1}^{d R} \longrightarrow \mathcal{U}^{\text {geom }}
$$


where $\mathcal{G}_{1,1}^{d R}$ is the de Rham relative completion of the fundamental group of $\mathfrak{M}_{1,1}$. One knows $[2,21]$ that the previous map factors through its quotient $\mathcal{U}_{E}^{d R}$, that is, all generators corresponding to cusp forms map to zero.

\subsection{Description of the map $\boldsymbol{\mu}$. The isomorphism}

$$
(\mathrm{X}, \mathrm{Y}) \mapsto(\mathrm{b}, \mathrm{a}): \mathbb{Q} \mathrm{X} \oplus \mathbb{Q} \mathrm{Y} \stackrel{\sim}{\longrightarrow} \mathbb{Q} \mathrm{b} \oplus \mathbb{Q} \mathrm{a}
$$

respects the action of $\mathrm{SL}_{2}^{d R}$ on both sides. This induces an isomorphism of the de Rham Lie algebras $\mathfrak{s l}_{2}$, which act on $\mathfrak{u}_{E}^{d R}$ and $\mathfrak{u}^{\text {geom }}$, respectively:

$$
\mathrm{X} \frac{\partial}{\partial \mathrm{Y}} \mapsto \varepsilon_{0}^{\vee} \quad \text { and } \quad-\mathrm{Y} \frac{\partial}{\partial \mathrm{X}} \mapsto \varepsilon_{0}
$$

DEFINITION 6.1. There is a unique morphism of Lie algebras satisfying

$$
\begin{aligned}
\mu: \mathfrak{u}_{E}^{d R} & \longrightarrow \mathfrak{u}^{\text {geom }} \\
\mathbf{e}_{2 n+2} \mathbf{X}^{2 n} & \mapsto \frac{2}{(2 n) !} \varepsilon_{2 n+2}
\end{aligned}
$$

for all $n \geqslant 1$, which is equivariant for the respective actions of $\mathfrak{s l}_{2}$ on both sides. In particular, $\mu\left(\left(\begin{array}{c}2 n \\ i\end{array}\right) \mathrm{X}^{2 n-i} \mathrm{Y}^{i}=\frac{2}{(2 n) !} \frac{(-1)^{i}}{i !} \mathrm{ad}\left(\varepsilon_{0}\right)^{i} \varepsilon_{2 n+2}\right.$. It respects the $M$ grading.

The map $\mu$ is surjective. It induces a surjective (faithfully flat) homomorphism of affine group schemes

$$
\mu: \mathcal{U}_{E}^{d R} \longrightarrow \mathcal{U}^{\text {geom }}
$$

On the level of complex points, it sends a group-like formal power series of the form

$$
\sum_{r \geqslant 0} a_{n_{1}, \ldots, n_{r}}^{i_{1}, \ldots, i_{r}}\left(\mathbf{e}_{2 n_{1}+2} \mathrm{X}^{i_{1}} \mathrm{Y}^{2 n_{1}-i_{1}} \otimes \cdots \otimes \mathbf{e}_{2 n_{r}+2} \mathrm{X}^{i_{r}} \mathrm{Y}^{2 n_{r}-i_{r}}\right)
$$

where $a_{n_{1}, \ldots, n_{r}}^{i_{1}, \ldots, i_{r}} \in \mathbb{C}$, to a series of the form (5.4) by applying $\mu \otimes \cdots \otimes \mu(r$ times) to each term of length $r$. For a series of the above type to be group-like is equivalent to certain shuffle product equations amongst the coefficients $a_{n_{1}, \ldots, n_{r}}^{i_{1}, \ldots, i_{r}}$. These are spelt out explicitly in [2, Section 3.6], where we used the slightly different 'multivariable' notation $\mathbf{e}_{2 n_{1}+2} \ldots \mathbf{e}_{2 n_{r}+2} \mathrm{X}_{1}^{i_{1}} \mathrm{Y}_{1}^{2 n_{1}-i_{1}} \ldots \mathrm{X}_{r}^{i_{r}} \mathrm{Y}_{r}^{2 n_{r}-i_{r}}$ instead of a tensor product of the form $\left(\mathbf{e}_{2 n_{1}+2} X^{i_{1}} Y^{2 n_{1}-i_{1}} \otimes \ldots \otimes \mathbf{e}_{2 n_{r}+2} X^{i_{r}} Y^{2 n_{r}-i_{r}}\right)$. 


\subsection{The generating series $J$}

Definition 6.2. Let $J$ denote the composition $\mu I^{E}$. It defines an analytic function

$$
J: \mathfrak{H} \longrightarrow \mathcal{U}^{\text {geom }}(\mathbb{C})
$$

whose coefficients are certain linear combinations of iterated integrals of $\mathbb{G}_{2 n}$, $n \geqslant 2$. For the explicit shape of $J(\tau)$, see equations (6.5) and the lines that follow. Note that only some, but not all, iterated integrals of Eisenstein series actually appear in $J$ because of the existence of relations in $\mathfrak{u}^{\text {geom }}$.

The following proposition is perhaps known to one or two experts, but is not stated explicitly in the literature to our knowledge.

Proposition 6.3. The function $J$ is the unique solution to the differential equation

$$
d J=-\omega J
$$

taking the value 1 at the tangent vector $\partial / \partial q$ at $q=0$, where $\omega$ is the formal 1-form

$$
\omega=-\operatorname{ad}\left(\varepsilon_{0}\right) \frac{d q}{q}+\sum_{n \geqslant 1} \frac{2}{(2 n) !} \varepsilon_{2 n+2} \mathbb{G}_{2 n+2}(q) \frac{d q}{q} .
$$

Proof. We shall use the following easily verified identity:

$$
(\mathrm{X}-\log (q) \mathrm{Y})^{n}=\exp \left(-\log (q) \mathrm{Y} \frac{\partial}{\partial \mathrm{X}}\right) \mathrm{X}^{n} .
$$

The map $\mu$ induces a map $\mu \otimes \mathrm{id}: \mathfrak{u}_{E}^{d R} \otimes \Omega^{1}(\mathfrak{H}) \longrightarrow \mathfrak{u}^{\text {geom }} \otimes \Omega^{1}(\mathfrak{H})$, which by definition (6.2) of $\mu$ and (4.2) of $\underline{E}_{2 n+2}(\tau)$ gives

$$
\begin{aligned}
(\mu \otimes \mathrm{id})\left(\mathbf{e}_{2 n+2} \underline{E}_{2 n+2}(\tau)\right) & =\frac{2}{(2 n) !} \exp \left(\log (q) \operatorname{ad}\left(\varepsilon_{0}\right)\right) \varepsilon_{2 n+2} \mathbb{G}_{2 n+2}(q) \frac{d q}{q} \\
& =\frac{2}{(2 n) !} \sum_{m \geqslant 0} \frac{1}{m !}(\log q)^{m} \operatorname{ad}\left(\varepsilon_{0}\right)^{m} \varepsilon_{2 n+2} \mathbb{G}_{2 n+2}(q) \frac{d q}{q} \\
& =\frac{2}{(2 n) !} \sum_{m \geqslant 0} \varepsilon_{2 n+2}^{(m)}(\int_{q}^{\overrightarrow{1}_{\infty}} \underbrace{\frac{d q}{q} \ldots \frac{d q}{q}}_{m}) \mathbb{G}_{2 n+2}(q) \frac{d q}{q}
\end{aligned}
$$

where for simplicity we write $\varepsilon_{2 n+2}^{(m)}$ for $\left(\operatorname{ad}\left(-\varepsilon_{0}\right)\right)^{m} \varepsilon_{2 n+2}$. These elements vanish whenever $m>2 n$ as a consequence of Lemma 5.2. 
The tangential base point $\overrightarrow{1}_{\infty}$ corresponds, in the $q$-disk, to $\frac{\partial}{\partial q}$, the unit tangent vector at the origin. From formula (4.4) for $I^{E}$ as an iterated integral transposed to the $q$-disk, we see that its image $\mu I^{E}$ has the form

$$
J=\mu I^{E}=\sum_{r \geqslant 0} \sum_{n_{1}, \ldots, n_{r} \geqslant 1} \sum_{m_{1}, \ldots, m_{r} \geqslant 0} J_{n_{1}, \ldots, n_{r}}^{m_{1}, \ldots, m_{r}}(q),
$$

where

$$
\begin{aligned}
& J_{n_{1}, \ldots, n_{r}}^{m_{1}, \ldots, m_{r}}(q)=\frac{2}{\left(2 n_{1}\right) !} \cdots \frac{2}{\left(2 n_{r}\right) !} \varepsilon_{2 n_{1}+2}^{\left(m_{1}\right)} \ldots \varepsilon_{2 n_{r}+2}^{\left(m_{r}\right)} \\
& \times \int_{q}^{\frac{\partial}{\partial q}}(\underbrace{\frac{d q}{q} \ldots \frac{d q}{q}}_{m_{1}} \mathbb{G}_{2 n_{1}+2}(q) \frac{d q}{q}) \ldots(\underbrace{\frac{d q}{q} \ldots \frac{d q}{q}}_{m_{r}} \mathbb{G}_{2 n_{r}+2}(q) \frac{d q}{q}) .
\end{aligned}
$$

It follows from the formula for the derivative of an iterated integral that

$$
d J_{n_{1}, \ldots, n_{r}}^{m_{1}, \ldots, m_{r}}(q)=\operatorname{ad}\left(\varepsilon_{0}\right) \frac{d q}{q} J_{n_{1}, n_{2}, \ldots, n_{r}}^{m_{1}-1, m_{2}, \ldots, m_{r}}(q)
$$

if $m_{1} \geqslant 1$, and

$$
d J_{n_{1}, \ldots, n_{r}}^{0, m_{2}, \ldots, m_{r}}(q)=-\frac{2}{\left(2 n_{1}\right) !} \varepsilon_{2 n_{1}+2} \mathbb{G}_{2 n_{1}+2}(q) \frac{d q}{q} J_{n_{2}, \ldots, n_{r}}^{m_{2}, \ldots, m_{r}}(q)
$$

otherwise. Therefore $J$ is the unique solution to the differential equation (6.3), which takes the value 1 at the unit tangent vector at the origin.

The image of $\mathcal{C}^{E}$ under the homomorphism $\mu$ is a non-Abelian cocycle

$$
\mathcal{G}:=\mu \mathcal{C}^{E} \in Z^{1}\left(\mathrm{SL}_{2}(\mathbb{Z}), \mathcal{U}^{\text {geom }}(\mathbb{C})\right)
$$

where $\mathrm{SL}_{2}(\mathbb{Z})$ acts via the Betti action (3.4). The function $J$ satisfies

$$
\left.J(\gamma \tau)\right|_{\gamma} \mathcal{G}_{\gamma}=J(\tau)
$$

for all $\tau \in \mathfrak{H}$, and $\mathcal{G}_{\gamma}$ satisfies the cocycle equation

$$
\mathcal{G}_{g h}=\left.\mathcal{G}_{g}\right|_{h} \mathcal{G}_{h}
$$

for all $g, h \in \mathrm{SL}_{2}(\mathbb{Z})$. 


\section{Action of complex conjugation}

Complex conjugation acts via $q \mapsto \bar{q}$ on the unit disk, and corresponds on the upper-half plane to the involution $\tau \mapsto-\bar{\tau}$.

It acts on $\mathcal{U}^{\text {geom }}(\mathbb{C})$ by complex conjugation on the coefficients. It is important to note that it does not respect the cocycle relation (6.8), since

$$
\overline{\mathcal{G}}_{g h}=\left.\overline{\mathcal{G}}_{g}\right|_{h} \overline{\mathcal{G}}_{h}
$$

and $\bar{h}$ is not in general equal to $h$ since the (Betti) action of $\mathrm{SL}_{2}(\mathbb{Z})$ on $\mathcal{U}^{\text {geom }}(\mathbb{C})$ is via (3.4), which involves powers of $2 \pi i$ (see (3.5)). To remedy this, we compose the action of complex conjugation with the element $-1 \in \mathbb{G}_{m}(\mathbb{Q})$, where $\mathbb{G}_{m}$ is the multiplicative group corresponding to the $M$-grading.

More precisely, consider the involution

$$
(a, b) \mapsto(-a, b): \mathbb{L}(a, b) \longrightarrow \mathbb{L}(a, b) .
$$

It has the effect of multiplying terms of $M$-degree $m$ by $(-1)^{m}$. This induces an involution $(-1): \mathfrak{u}^{\text {geom }} \rightarrow \mathfrak{u}^{\text {geom }}$ on the Lie subalgebra $\mathfrak{u}^{\text {geom }}$, and hence an involution $(-1): \mathcal{U}^{\text {geom }} \rightarrow \mathcal{U}^{\text {geom }}$ on affine group schemes.

Definition 7.1. Consider the homomorphism of groups

$$
\mathrm{sv}: \mathcal{U}^{\text {geom }}(\mathbb{C}) \longrightarrow \mathcal{U}^{\text {geom }}(\mathbb{C})
$$

which is obtained by composing $(-1): \mathcal{U}^{\text {geom }} \rightarrow \mathcal{U}^{\text {geom }}$ with the action of complex conjugation on coefficients, in either order (they commute).

It follows that the involution sv now preserves the cocycle relation

$$
\operatorname{sv} \mathcal{G}_{g h}=\operatorname{sv}\left(\left.\mathcal{G}_{g}\right|_{h}\right) \operatorname{sv}\left(\mathcal{G}_{h}\right)
$$

for all $g, h \in \mathrm{SL}_{2}(\mathbb{Z})$, and hence $\operatorname{sv} \mathcal{G} \in Z^{1}\left(\mathrm{SL}_{2}(\mathbb{Z}), \mathcal{U}^{\text {geom }}(\mathbb{C})\right)$. It was shown in [2] that the space of cocycles is a torsor over a certain automorphism group, and the technical heart of this paper is to relate $\mathcal{G}$ and sv $\mathcal{G}$ via this automorphism group. To state this, recall that $\mathcal{Z}^{\text {sv }} \subset \mathbb{R}$ denotes the ring of single-valued multiple zeta values. The following theorem involves a considerable amount of the machinery constructed in [2], and may be taken as a black box.

THEOREM 7.2. There exist elements

$$
b^{\mathrm{sv}} \in \mathcal{U}^{\text {geom }}\left(\mathcal{Z}^{\mathrm{sv}}\right) \quad \text { and } \quad \phi^{\mathrm{sv}} \in \operatorname{Aut}\left(\mathcal{U}^{\text {geom }}\right)^{\mathrm{SL}_{2}}\left(\mathcal{Z}^{\mathrm{sv}}\right)
$$


such that, for all $\gamma \in \mathrm{SL}_{2}(\mathbb{Z})$, we have

$$
\left.\left(b^{\mathrm{sv}}\right)^{-1}\right|_{\gamma} \phi^{\mathrm{sv}}\left(\mathrm{sv} \mathcal{G}_{\gamma}\right) b^{\mathrm{sv}}=\mathcal{G}_{\gamma} .
$$

The pair of elements $\left(b^{\mathrm{sv}}, \phi^{\mathrm{sv}}\right)$ is well-defined up to replacing it with $\left(a b^{\mathrm{sv}}\right.$, $\left.a \phi^{\mathrm{sv}} a^{-1}\right)$, where $a \in \mathcal{U}^{\mathrm{geom}}\left(\mathcal{Z}^{\mathrm{sv}}\right)^{\mathrm{SL}_{2}}$. Furthermore, the automorphism

$$
\left(b^{\text {sv }}\right)^{-1} \phi^{\text {sv }} b^{\text {sv }} \in \operatorname{Aut}\left(\mathcal{U}^{\text {geom }}\right)\left(\mathcal{Z}^{\text {sv }}\right)
$$

is induced by an automorphism $\psi^{\mathrm{sv}} \in \operatorname{Aut}(\Pi)\left(\mathcal{Z}^{\mathrm{sv}}\right)$ of $\Pi$ (defined in (5.1)).

The group $\operatorname{Aut}\left(\mathcal{U}^{\text {geom }}\right)^{\mathrm{SL}_{2}}$ denotes the $\mathrm{SL}_{2}$-invariant automorphisms of $\mathcal{U}^{\text {geom }}$, that is, automorphisms $\phi: \mathcal{U}^{\text {geom }} \stackrel{\sim}{\rightarrow} \mathcal{U}^{\text {geom }}$ satisfying $\phi(x g)=\phi(x) g$ for all $g \in$ $\mathrm{SL}_{2}^{d R}$, with the de Rham action on $\mathcal{U}^{\text {geom }}$. Therefore, an element $\phi \in \operatorname{Aut}\left(\mathcal{U}^{\text {geom }}\right)^{\mathrm{SL}_{2}}$ commutes with the (Betti) image of $\mathrm{SL}_{2}(\mathbb{Z})$ in $\mathrm{SL}_{2}^{d R}(\mathbb{C})$ under the comparison map (3.4).

7.1. Further properties. Before turning to the proof, we state a number of further properties satisfied by the elements $b^{\mathrm{sv}}, \phi^{\mathrm{sv}}$ of the theorem.

(i) The elements $b^{\mathrm{sv}}, \phi^{\mathrm{sv}}$ are, respectively, exponentials of elements

$$
\beta^{\text {sv }} \in \mathfrak{u}^{\text {geom }}\left(\mathcal{Z}^{\text {sv }}\right) \quad \text { and } \quad \delta^{\text {sv }} \in \operatorname{Der}\left(\mathfrak{u}^{\text {geom }}\right)^{\mathfrak{s l}_{2}}\left(\mathcal{Z}^{\text {sv }}\right) \text {, }
$$

where the derivation $\delta^{\mathrm{sv}}$ has the property that

$$
\left[\delta^{\mathrm{sv}}, \varepsilon_{0}\right]=0 \quad \text { and } \quad\left[\delta^{\mathrm{sv}}, \varepsilon_{0}^{\vee}\right]=0,
$$

which is equivalent to saying that $\delta^{\mathrm{sv}}$ is $\mathfrak{s l}_{2}$-invariant.

(ii) There exists a derivation

$$
\psi^{\mathrm{sv}}=\operatorname{Lie} \psi^{\mathrm{sv}} \in \operatorname{Der}^{\Theta} \operatorname{Lie}(\mathrm{a}, \mathrm{b})\left(\mathcal{Z}^{\mathrm{sv}}\right)
$$

which preserves $\mathfrak{u}^{\text {geom }}\left(\mathcal{Z}^{\text {sv }}\right)$ and whose restriction to $\mathfrak{u}^{\text {geom }}\left(\mathcal{Z}^{\text {sv }}\right)$ satisfies

$$
\left.\boldsymbol{\psi}^{\mathrm{sv}}\right|_{\mathfrak{u}^{\mathrm{geom}}\left(\mathcal{Z}^{\mathrm{sv}}\right)}=\operatorname{ad}\left(\beta^{\mathrm{sv}}\right)+\delta^{\mathrm{sv}} .
$$

This is a highly nontrivial constraint. For example, it expresses the nonobvious fact that the derivation on the right-hand side is uniquely determined by its action on a single element a (or b). Conversely, the fact that $\psi^{\text {sv }}$ in turn normalizes the image of all geometric derivations $\mathfrak{u}^{\text {geom }}$ is equivalent to an infinite sequence of combinatorial constraints of the form

$$
\left[\psi^{\mathrm{sv}}, \varepsilon_{2 n+2}\right] \in \mathfrak{u}^{\mathrm{geom}}\left(\mathcal{Z}^{\mathrm{sv}}\right)
$$


for every $n \geqslant 1$. It is far from obvious that there exist any solutions to these equations but follows from the Tannakian theory implicit in the proof of Theorem 7.2 (we know that 'motivic' derivations must satisfy a similar property).

(iii) Equation (7.2), in the case $\gamma=T$, is equivalent to the 'inertial relation'

$$
\left[\beta^{\mathrm{sv}}, \varepsilon_{0}\right]+\left[\beta^{\mathrm{sv}}, N_{+}\right]+\delta^{\mathrm{sv}}\left(N_{+}\right)=0,
$$

where the element $N_{+} \in \mathfrak{u}^{\text {geom }}$ is the element of $M$-degree -1 given by

$$
N_{+}=\sum_{n \geqslant 1} \frac{B_{2 n+2}}{4 n+4} \frac{2}{(2 n) !} \varepsilon_{2 n+2}
$$

and is the unipotent part of the logarithm of $\left(T, \mathcal{G}_{T}\right) \in \mathrm{SL}_{2} \ltimes \mathcal{U}^{\text {geom }}(\mathbb{C})$. The inertial relation ties together $\beta^{\text {sv }}$ and $\delta^{\text {sv }}$ : information about $\beta^{\text {sv }}$ can be deduced from information about $\delta^{\mathrm{sv}}$ and vice versa. Equation (7.2) is uniquely determined by its two instances $\gamma=T$ and $\gamma=S$. In other words, $\left(b^{\text {sv }}, \phi^{\text {sv }}\right)$ are uniquely determined (up to twisting by $a \in\left(\mathcal{U}^{\text {geom }}\right)^{\mathrm{SL}_{2}}$ via $(b, \phi) \mapsto\left(a b^{\mathrm{sv}}, a \phi^{\mathrm{sv}} a^{-1}\right)$ ), by the inertial relation and

$$
\left.\left(b^{\mathrm{sv}}\right)^{-1}\right|_{S} \phi^{\mathrm{sv}}\left(\mathrm{sv} \mathcal{G}_{S}\right) b^{\mathrm{sv}}=\mathcal{G}_{S} .
$$

(iv) It follows as a consequence of [2, Section 18] that to lowest order

$$
b \equiv 1+\sum_{n \geqslant 1} \zeta_{\mathrm{sv}}(2 n+1) \varepsilon_{2 n+2}^{\vee}\left(\bmod \left[\mathfrak{u}^{\mathrm{geom}}\left(\mathcal{Z}^{\mathrm{sv}}\right), \mathfrak{u}^{\mathrm{geom}}\left(\mathcal{Z}^{\mathrm{sv}}\right)\right]\right)
$$

is canonical, where $\zeta_{\mathrm{sv}}(2 n+1)=2 \zeta(2 n+1)$. The element $\phi^{\text {sv }}$ is also known to lowest order by [2, Theorem 16.9] via the inertial relation. In particular,

$$
\left[\delta^{\mathrm{sv}}, \mathfrak{u}^{\text {geom }}\left(\mathcal{Z}^{\mathrm{sv}}\right)\right] \subset\left[\mathfrak{u}^{\text {geom }}\left(\mathcal{Z}^{\mathrm{sv}}\right), \mathfrak{u}^{\text {geom }}\left(\mathcal{Z}^{\mathrm{sv}}\right)\right]
$$

The element $\psi^{\mathrm{sv}}$ satisfies $\psi^{\mathrm{sv}}(\mathrm{a}) \equiv \mathrm{a}$ and $\psi^{\mathrm{sv}}(\mathrm{b}) \equiv \mathrm{b}$ modulo terms of degree $\geqslant 3$ in b.

(v) The weights of the coefficients of $a, b^{\mathrm{sv}}, \phi^{\mathrm{sv}}$ are determined by the $M$ filtration. In fact, much more is true: replacing $b^{\mathrm{sv}}, \phi^{\mathrm{sv}}$ with their 'motivic' versions as in [2, Section 18.3], the action of the de Rham motivic Galois group of $\mathcal{M T}(\mathbb{Z})$ on their coefficients is determined by its action on $\left(b^{\mathrm{sv}}, \phi^{\mathrm{sv}}\right)$. Restricting to the subgroup $\mathbb{G}_{m}$ implies that the weights of the motivic multiple zeta values are induced by the $M$-grading on $\mathcal{O}\left(\mathcal{U}^{\text {geom }}\right)$.

(vi) Since complex conjugation is an involution, the elements sv and hence $\left(b^{\mathrm{sv}}, \phi^{\mathrm{sv}}\right)$ satisfy an involution equation, which we will not write down. 
REMARK 7.3. The element $\psi^{\mathrm{sv}} \in$ Aut $\Pi(\mathbb{C})$ is the image of the single-valued element sv defined below, and could be computed independently via the periods of $\Pi$. In particular, it is compatible [2] with the Hain morphism $\Phi$ from the motivic fundamental group of the projective line minus three points $\mathbb{P}^{1} \backslash\{0,1, \infty\}$. The image of sv in its group of automorphisms was computed in [4] and involves single-valued multiple zeta values. This provides yet another constraint, and hence a method to obtain information about $b^{\text {sv }}$ and $\phi^{\text {sv }}$, which we will not exploit here.

In conclusion, the elements $b^{\mathrm{sv}}, \phi^{\mathrm{sv}}$ are very heavily constrained and it should in principle be possible to compute them explicitly to higher orders.

7.2. Proof of Theorem 7.2. The proof follows closely the argument given in [2, Section 18], with some minor differences. We summarize the main ingredients, and refer to loc. cit. for further details.

(1) The objects $\mathcal{G}_{1,1}^{d R}, \pi_{1}^{d R}\left(\mathcal{E}_{\partial / \partial q}^{\times}, \overrightarrow{1}_{0}\right)$ are affine group schemes over $\mathbb{Q}$ whose affine rings are the de Rham components of Ind-objects in a category $\mathcal{H}$ of realizations. The objects in $\mathcal{H}$ consist of triples $\left(M_{B}, M_{d R}, c\right)$, where $M_{B}, M_{d R}$ are finite-dimensional $\mathbb{Q}$-vector spaces and $c$ is an isomorphism $M_{d R} \otimes_{\mathbb{Q}} \mathbb{C} \stackrel{\sim}{\rightarrow} M_{B} \otimes_{\mathbb{Q}} \mathbb{C}$. In addition, $M_{B}, M_{d R}$ are equipped with an increasing weight filtration (in our situation, the $M$-filtration) compatible with $c$, and $M_{d R}$ has a decreasing filtration $F$ such that $M_{B}$, equipped with the weight filtration and the filtration $c(F) \otimes \mathbb{C}$ on $M_{B} \otimes_{\mathbb{Q}} \mathbb{C}$, is a graded polarizable $\mathbb{Q}$-mixed Hodge structure. The final piece of data is a real Frobenius involution $F_{\infty}: M_{B} \stackrel{\sim}{\rightarrow} M_{B}$ compatible with $c$ and with complex conjugation on both $M_{B} \otimes \mathbb{C}$ and $M_{d R} \otimes \mathbb{C}$.

(2) By a Tannakian argument [21, Appendix B], one can choose splittings of the weight and Hodge filtrations $M$ and $F$ in the de Rham realization of $\mathcal{H}$. They are compatible with the monodromy homomorphism $\mu$. Therefore we can identify $\pi_{1}^{d R}\left(\mathcal{E}_{\partial / \partial q}^{\times}, \overrightarrow{1}_{0}\right)$ with $\Pi$ (see (5.1)). The image of $\mathcal{U}_{1,1}^{d R}$, the unipotent radical of $\mathcal{G}_{1,1}^{d R}$, under $\mu$ is by definition $\mathcal{U}^{\text {geom }}$ :

$$
\mu: \mathcal{U}_{1,1}^{d R} \longrightarrow \mathcal{U}^{\text {geom }} \leqslant \operatorname{Aut}(\Pi)
$$

(3) Let $G_{\mathcal{H}}^{d R}$ denote the group of tensor automorphisms of the fibre functor on the category $\mathcal{H}$, which sends $\left(M_{B}, M_{d R}, c\right)$ to $M_{d R}$. It is an affine group scheme over $\mathbb{Q}$. Consider the composition of isomorphisms

$$
M_{d R} \otimes \mathbb{C} \stackrel{c}{\longrightarrow} M_{B} \otimes \mathbb{C} \stackrel{F_{\infty}}{\longrightarrow} M_{B} \otimes \mathbb{C} \stackrel{c^{-1}}{\longrightarrow} M_{d R} \otimes \mathbb{C} .
$$


Since it is functorial in $M$, it defines a canonical element

$$
\mathbf{s} \in G_{\mathcal{H}}^{d R}(\mathbb{C}) .
$$

Thus for every object $X$ in $\mathcal{H}$, we deduce the existence of $\mathbf{s} \in \operatorname{Aut}\left(X^{d R}\right)(\mathbb{C})$, which is compatible with all morphisms in $\mathcal{H}$, and compute the action of the real Frobenius $F_{\infty}$ in the de Rham realization.

(4) The element $\mathbf{s}$ is canonical, but it is convenient to modify it as follows. Here our presentation differs slightly from that of [2, Section 19]. The action of $G_{\mathcal{H}}^{d R}$ on the de Rham component of the Lefschetz object $\mathbb{Q}(-1)=$ $(\mathbb{Q}, \mathbb{Q}, 1 \mapsto 2 \pi i)$ in $\mathcal{H}$ defines a morphism $\pi: G_{\mathcal{H}}^{d R} \rightarrow \operatorname{Aut}(\mathbb{Q})=\mathbb{G}_{m}$. The image of $\mathbf{s}$ under $\pi$ is $-1 \in \mathbb{C}^{\times}=\mathbb{G}_{m}(\mathbb{C})$. Now, the choice of splitting of the weight filtration $M$ is equivalent to an action of the multiplicative group $\mathbb{G}_{m}$ on the de Rham component of objects of $\mathcal{H}$, that is, a splitting of the homomorphism $\pi: G_{\mathcal{H}}^{d R} \rightarrow \mathbb{G}_{m}$. We now multiply $\mathbf{s}$ by the image of $-1 \in \mathbb{G}_{m}(\mathbb{Q})$ under this splitting to obtain a modified element $\mathrm{sv}=(-1) \mathbf{s} \in$ $G_{\mathcal{H}}^{d R}(\mathbb{C})$, which now acts by the identity on $\mathbb{Q}(-1)$. The element sv depends on the choice of splitting.

Note that since $\pi_{1}\left(\mathcal{E}_{\partial / \partial q}^{\times}, \overrightarrow{1}_{0}\right)$ has a mixed Tate Hodge structure, its $M$ filtration is canonically split in the de Rham realization by $F$, and the action of sv upon its complex points is canonical. The same applies for $\mathcal{U}^{\text {geom }}$, and so the statement of the theorem depends in no way on the choices of splittings.

(5) We therefore deduce the existence of an element

$$
\operatorname{sv} \in \operatorname{Aut}\left(\mathcal{G}_{1,1}^{d R}\right)(\mathbb{C}) \times \operatorname{Aut}(\Pi)(\mathbb{C}),
$$

which is compatible with $\mu$. It is the image of the element $\mathrm{sv} \in G_{\mathcal{H}}^{d R}$, which acts compatibly on both $\mathcal{G}_{1,1}^{d R}(\mathbb{C})$ and $\Pi(\mathbb{C})$. On the other hand, in [2, Section 10], we gave an explicit description of the group of automorphisms of $\mathcal{G}_{1,1}^{d R}$. We showed that, for any choice of splitting $\mathcal{G}_{1,1}^{d R} \cong \mathrm{SL}_{2} \ltimes \mathcal{U}_{1,1}^{d R}$, any automorphism of $\mathcal{G}_{1,1}^{d R}$ that acts trivially on $\mathrm{SL}_{2}$ defines a pair

$$
b \in \mathcal{U}_{1,1}^{d R}(\mathbb{C}) \quad \text { and } \quad \phi \in \operatorname{Aut}\left(\mathcal{U}_{1,1}^{d R}\right)^{\mathrm{SL}_{2}}(\mathbb{C}) .
$$

They are well-defined up to twisting by an element $a \in\left(\mathcal{U}_{1,1}^{d R}\right)^{\mathrm{SL}_{2}}(\mathbb{C})$. The (right) action of $(b, \phi)$ on $(g, u) \in\left(\mathrm{SL}_{2} \ltimes \mathcal{U}_{1,1}^{d R}\right)(\mathbb{C})$ is $\left(g,\left.b^{-1}\right|_{g} \phi(u) b\right)$. There exists, by the argument of [21, Appendix B], a splitting $\mathcal{G}_{1,1}^{d R} \cong \mathrm{SL}_{2} \ltimes \mathcal{U}_{1,1}^{d R}$ compatible with the choice of $M$-splittings. We deduce that the image of sv under the monodromy homomorphism $\mu$ is represented by a pair

$$
b^{\text {sv }} \in \mathcal{U}^{\text {geom }}(\mathbb{C}) \text { and } \quad \phi^{\text {sv }} \in \operatorname{Aut}\left(\mathcal{U}^{\text {geom }}\right)^{\mathrm{SL}_{2}}(\mathbb{C}),
$$


which are well-defined up to twisting by $a \in\left(\mathcal{U}^{\text {geom }}\right)^{\mathrm{SL}_{2}}(\mathbb{C})$. It follows from the compatibility of sv with $\mu$ that the image of sv $\in \operatorname{Aut}\left(\mathcal{U}^{\text {geom }}\right)(\mathbb{C})$ is given by the automorphism $\left(b^{\mathrm{sv}}\right)^{-1} \phi^{\mathrm{sv}} b^{\mathrm{sv}}$. It is induced by the automorphism sv $\in \operatorname{Aut}(\Pi)(\mathbb{C})$, which we call $\psi^{\mathrm{sv}}$ in the statement of the theorem.

(6) Now let us apply the element sv to the 'canonical cocycle'. The Betti component $\mathcal{G}_{1,1}^{B}$ of the relative completion $\mathcal{G}_{1,1}$ admits a natural map

$$
\pi_{1}^{\text {top }}\left(\mathcal{M}_{1,1}(\mathbb{C}), \overrightarrow{1}_{\infty}\right)=\mathrm{SL}_{2}(\mathbb{Z}) \longrightarrow \mathcal{G}_{1,1}^{B}(\mathbb{Q})
$$

This is one of the defining properties of relative completion. We deduce a map

$$
\mathrm{SL}_{2}(\mathbb{Z}) \longrightarrow \mathcal{G}_{1,1}^{B}(\mathbb{Q}) \stackrel{c}{\longrightarrow} \mathcal{G}_{1,1}^{d R}(\mathbb{C}) \cong \mathrm{SL}_{2}^{d R}(\mathbb{C}) \ltimes \mathcal{U}_{1,1}^{d R}(\mathbb{C}),
$$

where $c$ denotes the comparison isomorphism, $\operatorname{comp}_{B, d R}$ for short. The image of $\gamma \in \mathrm{SL}_{2}(\mathbb{Z})$ is $\left(c \gamma, \mathcal{C}_{\gamma}\right)$, where $c \gamma$ is the image of $\gamma$ under (3.4) and $\mathcal{C}_{\gamma} \in \mathcal{U}_{1,1}^{d R}(\mathbb{C})$ is called the canonical cocycle. Its image under $\mu$ is precisely $\mathcal{G}_{\gamma} \in \mathcal{U}^{\text {geom }}(\mathbb{C})$. Since $\gamma$ is Betti-rational, the action of $F_{\infty}$ corresponds, via the comparison isomorphism, to complex conjugation on coefficients of $c(\gamma)$. This action is computed by the element $\mathbf{s}$. The element $\mathrm{sv}$ computes complex conjugation composed with the map $-1 \in \mathbb{G}_{m}$ (7.1). Since the affine ring of $\mathrm{SL}_{2}$ is pure Tate, the latter action is trivial on $\mathrm{SL}_{2}$ and hence $\mathrm{sV}$ acts trivially on $\gamma$ (this is the reason for preferring $\mathrm{sv}$ over $\mathbf{s}$, which does not). It follows that the (right) action of sv satisfies the equation

$$
\left(\gamma, \mathrm{sv} \mathcal{G}_{\gamma}\right) \circ \mathrm{sv}=\left(\gamma, \mathcal{G}_{\gamma}\right) .
$$

Writing sv in terms of $\left(b^{\mathrm{sv}}, \phi^{\mathrm{sv}}\right)$ above, we deduce that

$$
\left.\left(b^{\mathrm{sv}}\right)^{-1}\right|_{\gamma} \phi^{\mathrm{sv}}\left(\mathrm{sv} \mathcal{G}_{\gamma}\right) b^{\mathrm{sv}}=\mathcal{G}_{\gamma} .
$$

(7) It remains to compute the coefficients of sv. For this, we need the fact that the motivic fundamental group of the punctured Tate elliptic curve (or rather, the image of $\mathcal{G}_{1,1}$ under the monodromy homomorphism $\mu$ ) is a mixed Tate motive over $\mathbb{Z}[20]$. It then follows from the results of [4] that, for any mixed Tate motive $M$ over $\mathbb{Z}$, the coefficients of sv in $\operatorname{Aut}\left(M_{d R}\right)(\mathbb{R})$ are singlevalued multiple zeta values.

The idea of this proof applies in a much more general setting. It may be possible to circumvent the final step (7), which appeals to deep results about the category of mixed Tate motives over $\mathbb{Z}$ by a direct argument following the procedure outlined in Remark 7.3. However, it is likely that this would forfeit some of the constraints described in Section 7.1, which would become conjectures given the current state of knowledge. 


\section{Equivariant iterated Eisenstein integrals}

We can now define modular-equivariant iterated integrals of Eisenstein series.

Definition 8.1. Let $\left(b^{\mathrm{sv}}, \phi^{\mathrm{sv}}\right)$ be as in Theorem 7.2. Then define

$$
J^{\mathrm{eqv}}(\tau)=J(\tau)\left(b^{\mathrm{sv}}\right)^{-1} \phi^{\mathrm{sv}}\left(\mathrm{sv} J(\tau)^{-1}\right) .
$$

It is well-defined up to right multiplication by an element $a \in\left(\mathcal{U}^{\text {geom }}\right)^{\mathrm{SL}_{2}}\left(\mathcal{Z}^{\text {sv }}\right)$.

THEOREM 8.2. The series $J^{\text {eqv }}$ defines a real-analytic function

$$
J^{\text {eqv }}: \mathfrak{H} \longrightarrow \mathcal{U}^{\text {geom }}(\mathbb{C})
$$

which is equivariant for the action of $\mathrm{SL}_{2}(\mathbb{Z})$ :

$$
\left.J^{\mathrm{eqv}}(\gamma \tau)\right|_{\gamma}=J^{\mathrm{eqv}}(\tau)
$$

Proof. Using the monodromy equation (6.7), we compute

$$
\begin{aligned}
\left.J^{\mathrm{eqv}}(\gamma \tau)\right|_{\gamma} & =\left.\left.J(\gamma \tau)\right|_{\gamma}\left(b^{\mathrm{sv}}\right)^{-1}\right|_{\gamma} \phi^{\mathrm{sv}}\left(\left.\operatorname{sv} J(\gamma \tau)^{-1}\right|_{\gamma}\right) \\
& =\left.J(\tau) \mathcal{G}_{\gamma}^{-1}\left(b^{\mathrm{sv}}\right)^{-1}\right|_{\gamma} \phi^{\mathrm{sv}}\left(\operatorname{sv} \mathcal{G}_{\gamma} \operatorname{sv} J(\tau)^{-1}\right) \\
& =J(\tau) \mathcal{G}_{\gamma}^{-1}\left(\left.\left(b^{\mathrm{sv}}\right)^{-1}\right|_{\gamma} \phi^{\mathrm{sv}}\left(\operatorname{sv} \mathcal{G}_{\gamma}\right) b^{\mathrm{sv}}\right)\left(b^{\mathrm{sv}}\right)^{-1} \phi^{\mathrm{sv}}\left(\operatorname{sv} J(\tau)^{-1}\right) \\
& =J(\tau)\left(b^{\mathrm{sv}}\right)^{-1} \phi^{\mathrm{sv}}\left(\operatorname{sv} J(\tau)^{-1}\right) \\
& =J^{\mathrm{eqv}}(\tau) .
\end{aligned}
$$

The cancellation of terms going from the third to the fourth equation follows from the defining property (7.2).

The series $J^{\text {eqv }}$ is of the form

$$
J^{\mathrm{eqv}}=J(\tau)(\operatorname{sv} J(\tau))^{-1}+\text { correction terms, }
$$

since $b^{\mathrm{sv}}$ and $\phi^{\mathrm{sv}}$ are to lowest order equal to 1 and the identity, respectively. Therefore, to leading orders, the coefficients of $J^{\text {eqv }}$ are the real or imaginary parts of iterated integrals of Eisenstein series of the appropriate length. The correction terms involve linear combinations of products of real and imaginary parts of iterated integrals of Eisenstein series of lower lengths with single-valued multiple zeta value coefficients. 
8.1. A digression: 'single-valued' versus 'equivariant'. The series $J^{\text {eqv }}(\tau)$ does not quite correspond to a naive 'single-valued version' of $J(\tau)$, which is

$$
J^{\mathrm{sv}}(\tau):=J(\tau)\left(b^{\mathrm{sv}}\right)^{-1} \phi^{\mathrm{sv}}\left(\mathrm{sv} J(\tau)^{-1}\right) b^{\mathrm{sv}}=J^{\mathrm{eqv}}(\tau) b^{\mathrm{sv}} .
$$

Unlike $J^{\text {eqv }}$, it is canonically defined, that is, does not depend on the choice of representative $\left(b^{\mathrm{sv}}, \phi^{\mathrm{sv}}\right)$. Its value at the unit tangent vector at the cusp is 1 :

$$
J^{\mathrm{sv}}\left(\overrightarrow{1}_{\infty}\right)=1
$$

The generating function $J^{\mathrm{sv}}(\tau)$ is not modular-equivariant. In fact, by the previous theorem, it satisfies

$$
\begin{aligned}
\left.J^{\mathrm{sv}}(\gamma \tau)\right|_{\gamma} & =\left.\left.J^{\mathrm{eqv}}(\gamma \tau)\right|_{\gamma} b^{\mathrm{sv}}\right|_{\gamma} \\
& =\left.J^{\mathrm{eqv}}(\tau) b^{\mathrm{sv}}\right|_{\gamma} \\
& =\left.J^{\mathrm{sv}}(\tau)\left(b^{\mathrm{sv}}\right)^{-1} b^{\mathrm{sv}}\right|_{\gamma}
\end{aligned}
$$

Thus if we define

$$
\mathcal{G}_{\gamma}^{\mathrm{sv}}=\left.\left(b^{\mathrm{sv}}\right)^{-1} b^{\mathrm{sv}}\right|_{\gamma} \in Z^{1}\left(\mathrm{SL}_{2}(\mathbb{Z}), \mathcal{U}^{\text {geom }}(\mathbb{C})\right)
$$

to be the single-valued cocycle, the single-valued generating series $J^{\text {sv }}$ satisfies

$$
\left.J^{\mathrm{sv}}(\gamma \tau)\right|_{\gamma}=J^{\mathrm{sv}}(\tau) \mathcal{G}_{\gamma}^{\mathrm{sv}} .
$$

The coefficients of $\mathcal{G}_{\gamma}^{\text {sv }}$ are single-valued multiple zeta values since this is true for $b^{\text {sv }}$. A key point is that the cocycle $\mathcal{G}^{\text {sv }}$ is a coboundary. It is for this reason that it can be trivialized (noncanonically) to produce a modular-equivariant function $J^{\text {eqv }}(\tau)$.

8.2. Properties of $\boldsymbol{J}^{\text {eqv }}$. Let $\left(b^{\text {sv }}, \phi^{\text {sv }}\right)$ be as in Theorem 7.2.

COROLLARY 8.3. The generating function $J^{\text {eqv }}$ satisfies the differential equation

$$
d J^{\mathrm{eqv}}=-\omega J^{\mathrm{eqv}}+J^{\mathrm{eqv}} \phi^{\mathrm{sv}}(\mathrm{sv} \omega) .
$$

It is the unique solution whose value at the tangent vector of length one at the cusp is

$$
J^{\mathrm{eqv}}\left(\overrightarrow{1}_{\infty}\right)=\left(b^{\mathrm{sv}}\right)^{-1}
$$


Proof. The differential equation follows from Definition 8.1 together with the observation that $J^{-1}$ satisfies the equation $d J^{-1}=J^{-1} \omega$. This follows from differentiating the equation $J^{-1} J=1$, which implies that $\left(d J^{-1}\right) J-J^{-1} \omega J=0$, and by multiplying on the right by $J^{-1}$. The formula for the value of $J^{\text {eqv }}$ at $\overrightarrow{1}_{\infty}$ is a consequence of the definition, the fact that $J\left(\overrightarrow{1}_{\infty}\right)=1$, and the fact that $\phi^{\mathrm{sv}}$, sv are group homomorphisms, and therefore preserve the identity in $\mathcal{U}^{\text {geom }}\left(\mathcal{Z}^{\text {sv }}\right)$.

In particular, the holomorphic component of the differential equation

$$
\left(\frac{\partial}{\partial \tau} J^{\mathrm{eqv}}\right) d \tau=-\omega J^{\mathrm{eqv}}
$$

is canonical, but the antiholomorphic part

$$
\left(\frac{\partial}{\partial \bar{\tau}} J^{\mathrm{eqv}}\right) d \bar{\tau}=J^{\mathrm{eqv}} \phi^{\mathrm{sv}}(\mathrm{sv} \omega)
$$

depends on the choice of $\phi^{\mathrm{sv}}$. The right-hand side is $J^{\mathrm{eqv}} \mathrm{Sv} \omega$ to leading order, and so $d J^{\text {eqv }} \equiv-\omega J^{\text {eqv }}+J^{\text {eqv }} \mathrm{sv} \omega$ modulo lower order terms.

LEMMA 8.4. Complex conjugation acts on $J^{\text {eqv }}$ via the formula

$$
\operatorname{sv}\left(b^{\mathrm{sv}} J^{\mathrm{eqv}}\right)=\widetilde{\phi}^{\mathrm{sv}}\left(J^{\mathrm{eqv}} b^{\mathrm{sv}}\right)^{-1},
$$

where $\widetilde{\phi}^{\mathrm{sv}}=\mathrm{sv} \phi^{\mathrm{sv}} \mathrm{sv}$.

Proof. The element $K=\mathrm{sv} J^{\text {eqv }}$ satisfies the equation

$$
d K=-\mathrm{sv}(\omega) K+K \widetilde{\phi}^{\mathrm{sv}}(\omega)
$$

and hence

$$
d K^{-1}=-\widetilde{\phi}^{\mathrm{sv}}(\omega) K^{-1}+K^{-1} \operatorname{sv}(\omega) .
$$

The element $F=\widetilde{\phi}^{\text {sv }}\left(J^{\text {eqv }}\right)$ satisfies the equation

$$
d F=-\widetilde{\phi}^{\mathrm{sv}}(\omega) F+F \widetilde{\phi}^{\mathrm{sv}}\left(\phi^{\mathrm{sv}}(\operatorname{sv}(\omega))\right)
$$

Since the holomorphic components of these two differential equations agree, it follows that $F=K^{-1} A$, for some $A: \mathfrak{H} \rightarrow \mathcal{U}^{\text {geom }}(\mathbb{C})$ that satisfies

$$
\left.A(\gamma \tau)\right|_{\gamma}=A(\tau) \quad \text { and } \quad \frac{\partial A}{\partial \tau}=0 .
$$

Its modular equivariance follows from the equivariance of $\phi^{\text {sv }}$ and the fact that sv preserves $\mathrm{SL}_{2}(\mathbb{Z})$. Consider any coefficient $a: \mathfrak{H} \rightarrow V_{2 n} \otimes \mathbb{C}$ of $A$. 
It defines a holomorphic section $\bar{a} \in \Gamma\left(\mathrm{SL}_{2}(\mathbb{Z}) \backslash \mathfrak{H} ; \mathbb{V}_{2 n}\right)$, where $\mathbb{V}_{2 n}$ denotes the vector bundle associated with $V_{2 n}$, which is analytic at the cusp. Such sections correspond to modular forms of weight $2 n$. On the other hand, from $A=K F$, we see that the coefficients of $A$ are linear combinations of iterated integrals of modular forms and their complex conjugates. By Corollary 4.5, $A$ is constant. We deduce that

$$
\mathrm{sv}\left(J^{\mathrm{eqv}}\right)=A \widetilde{\phi}^{\mathrm{sv}}\left(J^{\mathrm{eqv}}\right)^{-1}
$$

Multiplying by $\operatorname{sv}\left(b^{\text {sv }}\right)$ on the left and changing the constant $A$, this is equivalent to

$$
\operatorname{sv}\left(b^{\mathrm{sv}} J^{\mathrm{sv}}\left(b^{\mathrm{sv}}\right)^{-1}\right)=A^{\prime} \widetilde{\phi}^{\mathrm{sv}}\left(J^{\mathrm{sv}}\right)^{-1},
$$

where $J^{\text {sv }}=J^{\text {eqv }} b^{\text {sv }}$. But $J^{\text {sv }}\left(\overrightarrow{1}_{\infty}\right)=1$, which implies that $A^{\prime}=1$.

The previous result can also be deduced from the fact that complex conjugation and hence the elements $\mathbf{S}$ and $\mathrm{sv}$ in the proof of Theorem 7.2 are involutions. This implies a cumbersome identity involving $b^{\mathrm{sv}}, \phi^{\mathrm{sv}}$ and sv, which we chose to omit.

\section{Definition of a class of real-analytic modular forms}

Having defined a modular-equivariant function $J^{\text {eqv }}$, we can extract realanalytic modular forms from its coefficients, which in turn generate the space $\mathcal{M I}^{E}$.

9.1. Coefficients of $\boldsymbol{J}^{\text {eqv }}$. For every $\tau \in \mathfrak{H}$, we view $J^{\text {eqv }}(\tau) \in \mathcal{U}^{\text {geom }}(\mathbb{C})$ as a homomorphism $\mathcal{O}\left(\mathcal{U}^{\text {geom }}\right) \rightarrow \mathbb{C}$.

DEFINITION 9.1. We call a coefficient function an $\mathrm{SL}_{2}$-equivariant $\mathbb{Q}$-linear map

$$
c: \check{V}_{2 n}^{d R} \longrightarrow \mathcal{O}\left(\mathcal{U}^{\text {geom }}\right)
$$

Given such a function, consider the composite (all tensor products are over $\mathbb{Q}$ )

$$
\mathfrak{H} \stackrel{J^{\text {eqv }}}{\longrightarrow} \operatorname{Hom}\left(\mathcal{O}\left(\mathcal{U}^{\text {geom }}\right), \mathbb{C}\right) \stackrel{c}{\longrightarrow} \operatorname{Hom}\left(\check{V}_{2 n}^{d R}, \mathbb{C}\right) \cong V_{2 n} \otimes \mathbb{C}
$$

which we call the 'coefficient of $c$ in $J^{\text {eqv }}$ '. The last isomorphism is induced by the comparison $V_{2 n}^{d R} \otimes \mathbb{C} \cong V_{2 n} \otimes \mathbb{C}$ (3.3). The coefficient of $c$ is a real-analytic map

$$
c\left(J^{\text {eqv }}\right): \mathfrak{H} \longrightarrow V_{2 n} \otimes \mathbb{C} .
$$

Since $J^{\text {eqv }}$ is equivariant, it follows that for all $\gamma \in \mathrm{SL}_{2}(\mathbb{Z})$,

$$
\left.c\left(J^{\mathrm{eqv}}\right)(\gamma \tau)\right|_{\gamma}=c\left(J^{\mathrm{eqv}}\right) .
$$


9.2. Equivariant sections. The following result was proved in [6, Section 7].

Proposition 9.2. A real-analytic section $f: \mathfrak{H} \rightarrow V_{2 n} \otimes \mathbb{C}$

$$
f(\tau)=\sum_{r+s=2 n} f^{r, s}(\tau) X^{r} Y^{s}
$$

can be uniquely written in the form

$$
f(\tau)=\sum_{r+s=2 n} f_{r, s}(\tau)(X-\tau Y)^{r}(X-\bar{\tau} Y)^{s},
$$

where $f_{r, s}(\tau): \mathfrak{H} \rightarrow \mathbb{C}$ are real analytic. Then $f$ is equivariant if and only if the coefficients $f_{r, s}(\tau)$ are modular of weights $(r, s)$ for all $r, s$. If in addition the $f^{r, s}(\tau)$ admit expansions in the ring $\mathbb{C}[[q, \bar{q}]][\tau, \bar{\tau}]$, then $f_{r, s} \in P^{-(r+s)} \mathcal{M}_{r, s}$.

The proposition follows from applying the invertible change of variables

$$
\begin{aligned}
& X \mapsto \frac{\tau}{\tau-\bar{\tau}}(X-\bar{\tau} Y)-\frac{\bar{\tau}}{\tau-\bar{\tau}}(X-\tau Y) \\
& Y \mapsto \frac{1}{\tau-\bar{\tau}}(X-\bar{\tau} Y)-\frac{1}{\tau-\bar{\tau}}(X-\tau Y) .
\end{aligned}
$$

In the de Rham basis of $V_{2 n}^{d R}$, this corresponds to

$$
\begin{aligned}
& \mathrm{X} \mapsto \frac{\log (q)}{2 \mathbb{L}}(\mathrm{X}+\log (\bar{q}) \mathrm{Y})+\frac{\log (\bar{q})}{2 \mathbb{L}}(\mathrm{X}-\log (q) \mathrm{Y}) \\
& \mathrm{Y} \mapsto \frac{1}{2 \mathbb{L}}(\mathrm{X}+\log (\bar{q}) \mathrm{Y})-\frac{1}{2 \mathbb{L}}(\mathrm{X}-\log (q) \mathrm{Y})
\end{aligned}
$$

9.3. Definition of the space $\mathcal{M I}^{E}$. Define the modular components $c_{r, s}\left(J^{\text {eqv }}\right)$ of a coefficient function $c$ to be the functions defined in the manner of Proposition 9.2. They are the unique functions satisfying

$$
\sum_{r+s=2 n} c_{r, s}\left(J^{\mathrm{eqv}}\right)(X-\tau Y)^{r}(X-\bar{\tau} Y)^{s}=c\left(J^{\mathrm{eqv}}\right)(X, Y) \text {. }
$$

We shall show in Theorem 10.6 below that the $c_{r, s}\left(J^{\text {eqv }}\right)$ lie in $\mathcal{M}_{r, s}$.

Definition 9.3. Let $\mathcal{M I}^{E} \subset \mathcal{M}$ denote the $\mathcal{Z}^{\text {sv }}$-module generated by all modular components $c_{r, s}\left(J^{\text {eqv }}\right)$ of all coefficients of $J^{\text {eqv }}$. 
The space $\mathcal{M I}^{E}$ is well-defined, that is, independent of the choices $b^{\mathrm{sv}}, \phi^{\mathrm{sv}}$. This follows because $J^{\text {eqv }}$ is unique, by Theorem 7.2, up to multiplying on the right by an element $a \in\left(\mathcal{U}^{\text {geom }}\right)^{\mathrm{SL}_{2}}\left(\mathcal{Z}^{\mathrm{sv}}\right)$. The operation of multiplying on the right by such an element $a$ is equivalent to modifying the coefficients $c$ by linear combinations, with coefficients in $\mathcal{Z}^{\text {sv }}$, of coefficient functions of smaller length. Since $\mathcal{M} \mathcal{I}^{E}$ is a $\mathcal{Z}^{\text {sv }}$-module, it follows that the space generated by the coefficients of $J^{\text {eqv }}$ is unaffected by this procedure.

\section{First properties of $\mathcal{M} \mathcal{I}^{E}$}

Since the space $\mathcal{M} \mathcal{I}^{E}$ is generated by coefficients (9.1), it inherits a number of structures from the affine ring $\mathcal{O}\left(\mathcal{U}^{\text {geom }}\right)$. Some first examples are discussed here.

\subsection{Multiplicative structure}

PROPOSITION 10.1. The space $\mathcal{M} \mathcal{I}^{E}\left[\mathbb{L}, \mathbb{L}^{-1}\right]$ is closed under multiplication.

Proof. Since $\mathcal{O}\left(\mathcal{U}^{\text {geom }}\right)$ is a commutative ring, any two $\mathrm{SL}_{2}$-equivariant maps

$$
c: \check{V}_{2 m}^{d R} \longrightarrow \mathcal{O}\left(\mathcal{U}^{\text {geom }}\right) \quad \text { and } \quad c^{\prime}: \check{V}_{2 n}^{d R} \longrightarrow \mathcal{O}\left(\mathcal{U}^{\text {geom }}\right)
$$

can be multiplied together via

$$
\check{V}_{2 m}^{d R} \otimes \check{V}_{2 n}^{d R} \stackrel{c \otimes c^{\prime}}{\longrightarrow} \mathcal{O}\left(\mathcal{U}^{\text {geom }}\right) \otimes \mathcal{O}\left(\mathcal{U}^{\text {geom }}\right) \longrightarrow \mathcal{O}\left(\mathcal{U}^{\text {geom }}\right),
$$

where the second map is the multiplication on $\mathcal{O}\left(\mathcal{U}^{\text {geom }}\right)$. By composing with the inclusion of an isotypical factor in an $\mathrm{SL}_{2}$-equivariant decomposition

$$
\check{V}_{2 m+2 n-2 k}^{d R} \hookrightarrow \check{V}_{2 m+2 n}^{d R} \oplus \cdots \oplus \check{V}_{2|m-n|}^{d R} \cong \check{V}_{2 m}^{d R} \otimes \check{V}_{2 n}^{d R}
$$

of the left-hand side, we deduce that the product of coefficients $c, c^{\prime}$ can be decomposed as a linear combination of coefficients of the form

$$
c_{k}^{\prime \prime}: \check{V}_{2 m+2 n-2 k}^{d R} \longrightarrow \mathcal{O}\left(\mathcal{U}^{\text {geom }}\right)
$$

for $0 \leqslant k \leqslant \min \{m, n\}$. Since, furthermore, transformation (9.4) is linear with coefficients in $\mathbb{Q}\left[\mathbb{L}, \mathbb{L}^{-1}, \log q, \log \bar{q}\right]$, it follows that every product $c_{r, s} \cdot c_{r^{\prime}, s^{\prime}}^{\prime}$ of modular components (Section 9.3) can in turn be expressed as a linear combination, with coefficients in $\mathbb{Q}\left[\mathbb{L}, \mathbb{L}^{-1}, \log q, \log \bar{q}\right]$, of $c_{r+r^{\prime}, s+s^{\prime}}^{\prime \prime}$. In fact, the coefficients must lie in $\mathbb{Q}\left[\mathbb{L}, \mathbb{L}^{-1}\right]$, because any modular form satisfying (1.1) is translation-invariant, and so the coefficients in this formula must lie in the $T$ invariant subspace of $\mathbb{Q}\left[\mathbb{L}, \mathbb{L}^{-1}, \log q, \log \bar{q}\right]$, which is exactly $\mathbb{Q}\left[\mathbb{L}, \mathbb{L}^{-1}\right]$ (see $[6$, Lemma 2.2]). 
In particular, for any $r, s, r^{\prime}, s^{\prime} \geqslant 0$, multiplication on the space of real-analytic functions on the upper-half plane induces a map

$$
\mathcal{M I}_{r, s}^{E} \times \mathcal{M I}_{r^{\prime}, s^{\prime}}^{E} \subset \mathcal{M} \mathcal{I}^{E}\left[\mathbb{L}, \mathbb{L}^{-1}\right]_{r+r^{\prime}, s+s^{\prime}}
$$

In other words, the product of two functions in $\mathcal{M I}^{E}$ is again a linear combination of functions in $\mathcal{M} \mathcal{I}^{E}$ with coefficients in $\mathbb{Q}\left[\mathbb{L}, \mathbb{L}^{-1}\right]$ of the appropriate modular weights. Note that $\mathcal{M} \mathcal{I}^{E}$ itself is not an algebra. The examples in [6, Section 9.3] illustrate why powers of $\mathbb{L}$ are necessary. The two functions $F_{2,2}^{(0)}=\mathcal{E}_{2,0} \mathcal{E}_{0,2}+$ $\frac{1}{2} \mathcal{E}_{1,1}^{2}$ and $F_{0,0}^{(2)}=\mathbb{L}^{2}\left(\mathcal{E}_{2,0} \mathcal{E}_{0,2}-\frac{1}{4} \mathcal{E}_{1,1}^{2}\right)$ lie in $\mathcal{M I}^{E}$ and are linear combinations of products of functions in $\mathcal{M I}^{E}$ of length one, but to solve for $\mathcal{E}_{1,1}^{2}$, for example, one must invert $\mathbb{L}$.

10.2. Complex conjugation. The space $\mathcal{M I}^{E}$ is stable under the action of complex conjugation. This follows from the definition and Lemma 8.4, since the coefficients of $b^{\text {sv }}$ and $\phi^{\text {sv }}$ lie in the ring $\mathcal{Z}^{\text {sv }} \subset \mathcal{M} \mathcal{I}^{E}$.

10.3. Length filtration. The affine ring $\mathcal{O}\left(\mathcal{U}^{\text {geom }}\right)$ is equipped with an increasing length filtration $L$, which is dual to the lower central series on $\mathfrak{u}^{\text {geom }}$.

DEFINITION 10.2. This induces an increasing filtration

$$
\mathcal{M} \mathcal{I}_{\ell}^{E}=\left\langle c_{r, s}: \operatorname{Im}(c) \subseteq L_{\ell} \mathcal{O}\left(\mathcal{U}^{\text {geom }}\right)\right\rangle_{\mathcal{Z}^{\text {sv }}}
$$

Elements in $\mathcal{M I}_{\ell}^{E}$ are generated by the real and imaginary parts of iterated integrals of Eisenstein series of length less than or equal to $\ell$. The length filtration is compatible with the multiplicative structure defined above, since $L_{u} L_{v} \subset L_{u+v}$.

10.4. $M$-grading. The affine ring $\mathcal{O}\left(\mathcal{U}^{\text {geom }}\right)$ is nonnegatively graded by the $M$-degree, and furthermore, the $M$-degree is compatible with the action of $\mathrm{SL}_{2}$. In particular, the generators (6.1) of $\mathfrak{s l}_{2}$ have $M$-degrees $1,-1$, respectively.

Likewise, the space $V_{2 n}^{d R}$ inherits an $M$-grading with degrees $0,-1, \ldots,-2 n$ from (4.1). Its dual $\check{V}_{2 n}^{d R}$ has $M$-degrees $0,1, \ldots, 2 n$.

LEMMA 10.3. Every nonzero coefficient function

$$
c: \check{V}_{2 n}^{d R} \longrightarrow \mathcal{O}\left(\mathcal{U}^{\text {geom }}\right)
$$

is a map of $M$-graded $\mathrm{SL}_{2}$-modules of degree $m$, for some $m \geqslant 0$. It follows that its modular components $c_{r, s}$ inherit an $M$-grading from $\mathcal{O}\left(\mathcal{U}^{\text {geom }}\right)$ satisfying

$$
\operatorname{deg}_{M} c_{r, s}=\operatorname{deg}_{M} c=m \text { for all } r, s \geqslant 0 .
$$


Proof. The fact that a coefficient function respects the $M$-grading follows from the compatibility between the $M$-grading and $\mathrm{SL}_{2}$-action: any coefficient function is generated, under the action of $\mathrm{SL}_{2}$, by its image on a lowest weight vector. Since $\mathcal{O}\left(\mathcal{U}^{\text {geom }}\right)$ is concentrated in nonnegative $M$-degrees, and $\check{V}_{2 n}^{d R}$ has a component in $M$-degree 0 , it follows that any coefficient function must have degree $m \geqslant 0$.

Finally, we define $\operatorname{deg}_{M} 2 \pi i \tau=\operatorname{deg}_{M} 2 \pi i \bar{\tau}=1$. This implies that

$$
\operatorname{deg}_{M} \mathbb{L}=1 .
$$

With these definitions, the terms in (9.4) are homogeneous of degree zero, since $\operatorname{deg}_{M} X=0$ and $\operatorname{deg}_{M} Y=-1$. Therefore $\operatorname{deg}_{M} c_{r, s}=\operatorname{deg}_{M} c^{r, s}=\operatorname{deg}_{M} c$.

Note that the $M$-degree of $\mathbb{L}$ is compatible with the Hodge-theoretic weight of the function $2 \log |q|$, which is the single-valued period of a family of Kummer extensions, and has Hodge-theoretic weight 2. Recall that $M$ is normalized to be one half of the Hodge-theoretic weight. The motivic Lefschetz period that is associated with $2 \pi i$ also has Hodge-theoretic weight 2 , so these definitions are forced upon us from the fact that $\tau$ and $\bar{\tau}$ have $M$-degree zero.

DEFINITION 10.4. The $M$-grading defines an increasing filtration in the usual manner, and induces an $M$-filtration on $\mathcal{M \mathcal { I } ^ { E }}$, where the $M$-filtration on $\mathcal{Z}^{\text {sv }}$ is half the usual Hodge-theoretic weight filtration (the multiple zeta value weight).

The $M$-filtration is well-defined (independent of the choice of representatives $b^{\text {sv }}, \phi^{\text {sv }}$ in Theorem 7.2) since it is induced by the $M$-filtration on $\mathcal{O}\left(\mathcal{U}^{\text {geom }}\right)$. Another way to say this is that the coproduct on $\mathcal{O}\left(\mathcal{U}^{\text {geom }}\right)$ dual to multiplication in $\mathcal{U}^{\text {geom }}$ is compatible with the $M$-filtration, so twisting by an element $a$, as in Theorem 7.2, only modifies the coefficient functions by elements of lower $M$ weight.

REMARK 10.5. Conjecturally, the weight filtration is a grading on $\mathcal{Z}^{\text {sv }}$. One way to exploit this is to work with motivic periods instead of real numbers, since motivic single-valued multiple zeta values are indeed weight-graded. This amounts to replacing $b^{\text {sv }}, \phi^{\text {sv }}$ with their 'motivic' versions, defined in [2]. Then the weight grading on the motivic periods is precisely dual to the $M$-grading on $\mathcal{O}\left(\mathcal{U}^{\text {geom }}\right)$. It remains to verify that this grading is not disturbed when extracting coefficients and modular components (Section 9.3). To see this, note that the comparison isomorphism (3.3) must be replaced with the universal comparison isomorphism in which one replaces $2 \pi i$ by its motivic version $(2 \pi i)^{\mathfrak{m}}$, which is Tate, and $M$-graded of degree 1 . The motivic version of the change of variables (9.4), in which one replaces $2 \pi i$ by $(2 \pi i)^{\mathfrak{m}}$, is therefore homogeneous of total 
degree 0 , which implies that $\operatorname{deg}_{M} c_{r, s}=\operatorname{deg}_{M} c$. It follows that the $M$-grading on $\mathcal{O}\left(\mathcal{U}^{\text {geom }}\right)$ induces an $M$-grading on all coefficient functions. This leads to a class of 'motivic' modular forms, which are formal expansions (1.4) whose coefficients are motivic periods (in this case, motivic single-valued multiple zeta values). Their images under the period homomorphism are genuine modular forms in $\mathcal{M}$. This will be discussed elsewhere. Note that the $M$-grading can be used to determine the powers of $\mathbb{L}$ on the right-hand side of (10.1).

Since the generators of $\mathfrak{u}^{\text {geom }}$ have strictly negative $M$-degrees and since $M_{0} \mathcal{Z}^{\text {sv }} \cong \mathbb{Q}$, we deduce that $M_{0} \mathcal{M} \mathcal{I}^{E}=\mathbb{Q}$.

\subsection{Expansions and coefficients}

THEOREM 10.6. Every element of $\mathcal{M I}^{E}$ admits an expansion in the ring

$$
\mathcal{Z}^{\mathrm{sv}}[[q, \bar{q}]]\left[\mathbb{L}, \mathbb{L}^{-1}\right],
$$

that is, its coefficients are single-valued multiple zeta values. An element of total modular weight $w$ has poles in $\mathbb{L}$ of order at most $w$.

Proof. By Theorem 7.2, the coefficients of $b^{\text {sv }}, \phi^{\text {sv }}$ are in the ring $\mathcal{Z}^{\text {sv }}$. It follows from the definition of $J^{\text {eqv }}$ that its coefficients are $\mathcal{Z}^{\text {sv }}$-linear combinations of real and imaginary parts of the coefficients of $J$. By Proposition 4.2, the latter are iterated integrals with expansions in the ring $\mathbb{Q}[[q]][\log q]$. Finally, the change of variables (9.4) is defined over $\mathbb{Q}\left[\mathbb{L}, \mathbb{L}^{-1}, \log q, \log \bar{q}\right]$. This proves that every element of $\mathcal{M I}^{E}$ admits an expansion in

$$
\mathcal{Z}^{\mathrm{sv}}[[q, \bar{q}]]\left[\mathbb{L}, \mathbb{L}^{-1}, \log q, \log \bar{q}\right] .
$$

A modular form is translation-invariant, so its expansion lies in the subring of $T$-invariants, which is precisely $\mathcal{Z}^{\text {sv }}[[q, \bar{q}]]\left[\mathbb{L}, \mathbb{L}^{-1}\right]$ (see [6, Lemma 2.2]). The bound on the order of poles in $\mathbb{L}$ follows from the definition of the coefficients and (9.4).

\subsection{Finiteness}

THEOREM 10.7. The subspace of elements of $\mathcal{M I}^{E}$ of total modular weight $w$ and $M$-degree $\leqslant m$ is finite-dimensional for every $m, w$.

Proof. It is enough to show that the dimension of each $\mathrm{SL}_{2}$-isotypical component of $\operatorname{gr}_{m}^{M} \mathcal{O}\left(\mathcal{U}^{\text {geom }}\right)$, or equivalently $\operatorname{gr}_{-m}^{M} \mathfrak{u}^{\text {geom }}$, is finite-dimensional. Since the latter 
is a quotient of $\mathfrak{u}_{E}^{d R}$, it is enough to prove it for the free Lie algebra on $\mathbf{e}_{2 n+2} V_{2 n}^{d R}$. Note in passing that since $\operatorname{gr}_{M} \mathbf{e}_{2 n+2}=-1$, the space $\operatorname{gr}_{-m}^{M} \mathfrak{u}_{E}^{d R}$ only involves Lie words of bounded length in the $\mathbf{e}_{2 n+2} V_{2 n}^{d R}$. Now the inclusion of an isotypical component

$$
V_{2 \ell}^{d R} \longrightarrow V_{2 n_{1}}^{d R} \otimes \cdots \otimes V_{2 n_{r}}^{d R}
$$

has $M$-degree $\ell-\left(n_{1}+\cdots+n_{r}\right)$. This follows from the fact that the map $\delta_{d R}^{k}$ defined in (3.1) has $M$-degree $k$, so its dual has $M$-degree $-k$, where $k=n_{1}+\cdots+$ $n_{r}-\ell$. The integer $\ell$ is constrained by the modular weight: $\ell=w$. The statement follows from the fact that the number of strictly positive integers $n_{1}, \ldots, n_{r}$ whose sum is bounded above is finite, and that the subring of $\mathcal{Z}^{\text {sv }}$ of bounded weight is finite-dimensional.

10.7. Compatibilities. The above structures are all mutually compatible: for example, the length filtration and $M$-filtration are compatible with the algebra structure.

\section{Differential structure of $\mathcal{M} \mathcal{I}^{E}$}

The ordinary differential equations satisfied by $J^{\text {eqv }}$ are equivalent to a system of differential equations involving the operators $\partial$ and $\bar{\partial}$ satisfied by its modular components. These in turn give rise to inhomogeneous Laplace eigenvalue equations.

\subsection{Vector-valued differential equations}

Proposition 11.1. Let $F, A, B: \mathcal{H} \rightarrow V_{2 n} \otimes \mathbb{C}$ be real analytic. Then the equation

$$
\frac{\partial F}{\partial z}=\frac{2 \pi i}{2} A(z)
$$

is equivalent to the following system of equations:

$$
\begin{aligned}
\partial F_{2 n, 0} & =\mathbb{L} A_{2 n, 0} \\
\partial F_{r, s}-(r+1) F_{r+1, s-1} & =\mathbb{L} A_{r, s} \quad \text { if } s \geqslant 1,
\end{aligned}
$$

for all $r+s=2 n$, and $r, s \geqslant 0$. In a similar manner,

$$
\frac{\partial F}{\partial \bar{z}}=\frac{2 \pi i}{2} B(z)
$$


is equivalent to the following system of equations:

$$
\begin{aligned}
\bar{\partial} F_{0,2 n} & =\mathbb{L} B_{0,2 n} \\
\bar{\partial} F_{r, s}-(s+1) F_{r-1, s+1} & =\mathbb{L} B_{r, s} \quad \text { if } r \geqslant 1 .
\end{aligned}
$$

The proof is a straightforward computation [6, Section 7].

Lemma 11.2. Suppose that $A: \mathfrak{H} \rightarrow V_{2 n} \otimes \mathbb{C}$ and set

$$
F=\frac{\delta_{d R}^{k}}{(k !)^{2}}\left((X-z Y)^{2 m} \otimes A\right) .
$$

Then $F: \mathfrak{H} \rightarrow V_{2 m+2 n-2 k} \otimes \mathbb{C}$ vanishes if $k>2 n$ or $k>2 m$, and its components satisfy

$$
F_{r, s}=(2 \mathbb{L})^{k}\left(\begin{array}{c}
2 m \\
k
\end{array}\right)\left(\begin{array}{c}
s+k \\
k
\end{array}\right) A_{r-2 m+k, s+k},
$$

where we set $A_{p, q}=0$ for $p<0$ or $q<0$. Therefore $F_{r, s}$ vanishes if $r<2 m-k$, or equivalently, $s+k>2 n$.

Proof. The statement with $\delta_{d R}$ replaced by $\delta$ is given in [6, Section 7]. Use $\delta_{d R}=2 \pi i \delta$.

11.2. Differential structure of $\mathcal{M I}^{E}$. Recall that the graded $\mathbb{Q}[\mathbb{L}]$-vector space generated by Eisenstein series is denoted by

$$
E=\bigoplus_{m \geqslant 1, n \geqslant 0} \mathbb{G}_{2 m+2} \mathbb{L}^{n} \mathbb{Q} .
$$

The terms $\mathbb{G}_{2 m+2}$ are assigned an $M$-degree of zero.

THEOREM 11.3. Every modular component $c_{r, s}$ of a coefficient function $c$ satisfies

$$
\partial c_{r, s}-(r+1) c_{r+1, s-1} \in E \times \mathcal{M I}_{k-1}^{E},
$$

where we define $c_{r, s}$ to be zero if $r$ or $s$ is negative. Similarly,

$$
\bar{\partial} c_{r, s}-(s+1) c_{r-1, s+1} \in \bar{E} \times \mathcal{M I}_{k-1}^{E} .
$$

In both these equations, the products on the right-hand sides denote their images under multiplication inside the ring of real-analytic functions on the upper-half plane.

Proof. Let $c: \check{V}_{2 n}^{d R} \rightarrow \mathcal{O}\left(\mathcal{U}^{\text {geom }}\right)$ be a coefficient. Embed $\mathcal{O}\left(\mathcal{U}^{\text {geom }}\right) \subset \mathcal{O}\left(\mathcal{U}_{E}^{d R}\right)$ via $\mu^{*}$, where $\mu$ is the monodromy homomorphism. We shall also denote the induced 
$\mathrm{SL}_{2}$-equivariant map $\mu^{*} c: \check{V}_{2 n}^{d R} \rightarrow \mathcal{O}\left(\mathcal{U}_{E}^{d R}\right)$ by $c$ and view a coefficient function as taking values in $\mathcal{O}\left(\mathcal{U}^{\text {geom }}\right)$ or $\mathcal{O}\left(\mathcal{U}_{E}^{d R}\right)$ as appropriate. If we choose any splitting $\mathcal{O}\left(\mathcal{U}_{E}^{d R}\right) \rightarrow \mathcal{O}\left(\mathcal{U}^{\text {geom }}\right)$ as $\mathrm{SL}_{2}$-modules, we can write $J^{\text {eqv }}$ via Definition 8.1 in the form

$$
J^{\mathrm{eqv}}(\tau)=\mu\left(I^{E} \bar{K}\right)
$$

for some antiholomorphic function $\bar{K}: \mathfrak{H} \rightarrow \mathcal{U}^{\text {geom }}(\mathbb{C})$, which is viewed as a function taking values in $\widehat{\mathcal{U}}_{E}^{d R}(\mathbb{C})$ via our choice of splitting. Therefore

$$
\frac{\partial}{\partial \tau} c\left(J^{\mathrm{eqv}}\right)=-c\left(\mu\left(\widetilde{\Omega}^{E} I^{E} \bar{K}\right)\right)
$$

where

$$
\widetilde{\Omega}^{E}=2 \pi i \sum_{n \geqslant 1} \mathbf{e}_{2 n+2} \mathbb{G}_{2 n+2}(\tau)(X-\tau Y)^{2 n},
$$

which follows from the differential equation $d I^{E}=-\Omega^{E} I^{E}$ and (4.3). The coproduct

$$
\Delta: \mathcal{O}\left(\mathcal{U}_{E}^{d R}\right) \longrightarrow \mathcal{O}\left(\mathcal{U}_{E}^{d R}\right) \otimes \mathcal{O}\left(\mathcal{U}_{E}^{d R}\right)
$$

dual to the multiplication law in $\mathcal{U}_{E}^{d R}$ is $\mathrm{SL}_{2}$-equivariant. In particular, it induces a coproduct on coefficient functions $c: \check{V}_{2 n}^{d R} \rightarrow \mathcal{O}\left(\mathcal{U}_{E}^{d R}\right)$ in the following form (using a variant of Sweedler's notation):

$$
\Delta c=\sum_{k \geqslant 0}\left(c^{\prime} \otimes c^{\prime \prime}\right)\left(\delta_{d R}^{k}\right)^{\vee}
$$

where $\left(\delta_{d R}^{k}\right)^{\vee}: \check{V}_{2 n}^{d R} \subset \check{V}^{d R} \rightarrow \check{V}^{d R} \otimes \check{V}^{d R}$ is the dual of $\delta_{d R}^{k}: V^{d R} \otimes V^{d R} \rightarrow V^{d R}$, and $V^{d R}=\bigoplus_{n \geqslant 0} V_{n}^{d R}=\mathbb{Q}[\mathrm{X}, \mathrm{Y}]$. Let $\langle$,$\rangle denote the pairing between the points$ of $\mathcal{U}^{\text {geom }}$ and $\mathcal{O}\left(\mathcal{U}^{\text {geom }}\right)$. We can rewrite our differential equation in the form

$$
\frac{\partial}{\partial \tau} c\left(J^{\mathrm{eqv}}\right)=-\left\langle c, m(\mu \otimes \mu)\left(\widetilde{\Omega}^{E} \otimes I^{E} \bar{K}\right)\right\rangle,
$$

where $m$ denotes multiplication, and $\widetilde{\Omega}^{E}$ is viewed as a section of $L_{1} \mathcal{O}\left(\mathcal{U}_{E}^{d R}\right)$. By the duality between coproducts and multiplication, this equals

$$
\begin{aligned}
\frac{\partial}{\partial \tau} c\left(J^{\mathrm{eqv}}\right) & =-\left\langle\Delta c,(\mu \otimes \mu)\left(\widetilde{\Omega}^{E} \otimes I^{E} \bar{K}\right)\right\rangle \\
& =-\sum_{k \geqslant 0}\left\langle\left(c^{\prime} \otimes c^{\prime \prime}\right)\left(\delta_{d R}^{k}\right)^{\vee}, \widetilde{\Omega}^{E} \otimes J^{\mathrm{eqv}}\right\rangle \\
& =-\sum_{k \geqslant 0} \delta_{d R}^{k}\left(\left(c^{\prime} \otimes c^{\prime \prime}\right)\left(\widetilde{\Omega}^{E} \otimes J^{\mathrm{eqv}}\right)\right),
\end{aligned}
$$


where in the second line, we view $c^{\prime \prime}$ as a coefficient function on $\mathcal{O}\left(\mathcal{U}^{\text {geom }}\right)$ and $c^{\prime}$ as a coefficient function on $\mathcal{O}\left(\mathcal{U}_{E}^{d R}\right)$. Restricted to $L_{1} \mathcal{O}\left(\mathcal{U}_{E}^{d R}\right)$, the map $c^{\prime}$ is a linear combination of the maps that send one $\mathrm{e}_{2 r+2}$ to 1 and all other $\mathrm{e}_{2 r^{\prime}+2}$ to 0 . We conclude that

$$
\frac{\partial}{\partial \tau} c\left(J^{\mathrm{eqv}}\right)=2 \pi i A,
$$

where $A: \mathfrak{H} \rightarrow V_{2 n} \otimes \mathbb{C}$ is a $\mathbb{Q}$-linear combination of terms of the form

$$
\delta_{d R}^{k}\left(\mathbb{G}_{2 m+2}(\tau)(X-\tau Y)^{2 m} \otimes c^{\prime \prime}\left(J^{\mathrm{eqv}}\right)\right),
$$

where $c^{\prime \prime}$ is a coefficient function of strictly smaller length than $c$, since the coproduct $\Delta$ is compatible with the length filtration. The first part of the theorem follows on applying Proposition 11.1 and Lemma 11.2. The second part follows from the first using the fact that $\mathcal{M I}^{E}$ is stable under complex conjugation.

Since $\mathcal{M I}^{E}$ is generated by coefficient functions, we deduce the following corollary.

COROLLARY 11.4. The space $\mathcal{M I}^{E}$ has the following differential structure:

$$
\begin{aligned}
& \partial\left(\mathcal{M I}_{k}^{E}\right) \subset \mathcal{M I}_{k}^{E}+E \times \mathcal{M I}_{k-1}^{E} \\
& \bar{\partial}\left(\mathcal{M I}_{k}^{E}\right) \subset \mathcal{M I}_{k}^{E}+\bar{E} \times \mathcal{M I}_{k-1}^{E} .
\end{aligned}
$$

The operators $\partial, \bar{\partial}$ respect the $M$-filtration.

Proof. The first part is immediate from the previous theorem. The statement about the $M$-filtration follows since the coproduct $\Delta$ respects the $M$-grading on $\mathcal{O}\left(\mathcal{U}_{E}^{d R}\right)$, the Eisenstein series $\mathbb{G}_{2 m+2}$ lie in $M$-degree zero, and the fact that in Lemma 11.2, the $M$-degree of the powers of $\mathbb{L}$ matches the $M$-degree of $\delta_{d R}^{k}$.

By (2.6), an element $\xi \in \mathcal{M}_{r, s}$ is uniquely determined by $\partial \xi$ and $\bar{\partial} \xi$, up to a possible multiple of $\mathbb{L}^{-r}$ in the case $r=s$. When $\xi \in \mathcal{M \mathcal { I } ^ { E }}$, this constant is an element of $\mathcal{Z}^{\text {sv }}$, whose $M$-weight can be determined from the $M$-grading.

REMARK 11.5. By the independence of iterated integrals (Corollary 4.5), the sums on the right-hand side in the previous corollary are direct, and so we may write

$$
\partial\left(\mathcal{M I}_{k}^{E}\right) \subset \mathcal{M I}_{k}^{E} \oplus\left(E \times \mathcal{M I}_{k-1}^{E}\right)
$$

and similarly for $\bar{\partial}$. This is because $E$ does not contain any constant functions. 
11.3. Reconstruction of vector-valued modular forms. Given an element $f_{m, n} \in \mathcal{M I}_{k}^{E}$ of modular weights $m, n \geqslant 0$, we can use the splitting of Remark 11.5 to define functions $f_{r, s} \in \mathcal{M I}_{k}^{E}$ for all $m+n=r+s$ and $r, s \geqslant 0$ via

$$
\partial f_{r, s}=(r+1) f_{r+1, s-1}\left(\bmod E \times \mathcal{M I}_{k-1}^{E}\right)
$$

whenever $s>1$ (since $r+1 \neq 0)$ and via

$$
\bar{\partial} f_{r, s}=(s+1) f_{r-1, s+1}\left(\bmod \bar{E} \times \mathcal{M I}_{k-1}^{E}\right)
$$

whenever $r>1$ (since $s+1 \neq 0$ ). That these equations are consistent follows from $[\partial, \bar{\partial}]=h$. The function

$$
F(\tau)=\sum_{r, s} f_{r, s}(X-\tau Y)^{r}(X-\bar{\tau} Y)^{s}
$$

is then a vector-valued modular form, and can be reconstructed from any one of its individual modular components $f_{m, n}$.

11.4. Laplace operator for vector-valued functions. The following lemma explains the existence of Laplace eigenvalue equations in a general setting.

LEMMA 11.6. Let $F: \mathfrak{H} \rightarrow V_{2 n} \otimes \mathbb{C}$ be real analytic satisfying the equation

$$
d F=\frac{(2 \pi i)}{2}(A d z+B d \bar{z})
$$

for some $A, B: \mathfrak{H} \rightarrow V_{2 n} \otimes \mathbb{C}$. Then

$$
\begin{aligned}
-(\Delta+r+s) F_{r, s} & =\mathbb{L}\left(\bar{\partial} A_{r, s}+(r+1) B_{r+1, s-1}\right) \\
& =\mathbb{L}\left(\partial B_{r, s}+(s+1) A_{r-1, s+1}\right),
\end{aligned}
$$

where $A_{r, s}, B_{r, s}$ are understood to be zero if any subscript $r$ or $s$ is negative.

Proof. The differential equation $d^{2} F=0$ implies that $\frac{\partial B}{\partial z}-\frac{\partial A}{\partial \bar{z}}=0$. By Proposition 11.1, this is equivalent to the equation

$$
\partial B_{r, s}-(r+1) B_{r+1, s-1}=\bar{\partial} A_{r, s}-(s+1) A_{r-1, s+1}
$$

for all $r+s=2 n$, This shows that the two expressions (11.8) are equivalent. Again by Proposition 11.1 and the relation $[\bar{\partial}, \mathbb{L}]=0$, we verify that

$$
\begin{aligned}
\bar{\partial} \partial F_{r, s} & =(r+1) \bar{\partial} F_{r+1, s-1}+\mathbb{L} \bar{\partial} A_{r, s} \\
& =(r+1) s F_{r, s}+\mathbb{L} \bar{\partial} A_{r, s}+(r+1) \mathbb{L} B_{r+1, s-1} .
\end{aligned}
$$

The statement follows from the definition of the Laplacian (2.7). 


\subsection{Laplace operator structure for $\mathcal{M} \mathcal{I}^{E}$.}

COROLLARY 11.7. Every element $F \in \mathcal{M I}_{k}^{E}$ of modular weights $(r, s)$ satisfies an inhomogeneous Laplace equation of the following form:

$$
(\Delta+r+s) F \in(E+\bar{E}) \times \mathcal{M I}_{k-1}^{E}+E \bar{E} \times \mathcal{M I}_{k-2}^{E},
$$

where the eigenvalue is minus the total modular weight. Recall that $E$ is the $\mathbb{Q}$ vector space spanned by $\mathbb{L}^{i} \mathbb{G}_{2 m+2}$ for $i \geqslant 0, m \geqslant 1$ and $\bar{E}$ is its complex conjugate. The space $E \bar{E}$ is generated by $\mathbb{L}^{i} \mathbb{G}_{2 m+2} \overline{\mathbb{G}}_{2 n+2}$ for $i \geqslant 0, m, n \geqslant 1$.

Proof. This follows from the previous lemma and equations (11.6) and (11.7) (or by direct application of the definition of the Laplace operator, using these same two equations and the Leibniz rule).

The sum on the right-hand side is direct by Corollary 4.5. It could be written as

$$
\left(E \times \mathcal{M I}_{k-1}^{E}\right) \oplus\left(\bar{E} \times \mathcal{M I}_{k-1}^{E}\right) \oplus\left(E \bar{E} \times \mathcal{M I}_{k-2}^{E}\right) .
$$

\section{Algebraic structure of $\mathcal{M} \mathcal{I}^{E}$}

In this section, we delve more deeply into the algebraic structure of $\mathcal{M I}^{E}$. Since the space $\mathcal{M} \mathcal{I}^{E}$ is generated from the coefficients of $\mathcal{O}\left(\mathcal{U}^{\text {geom }}\right)$, its structure is closely related to that of the geometric Lie algebra $\mathfrak{u}^{\text {geom }}$. Although the precise structure of the latter is not completely known, we can use the relationship with $\mathcal{M I}^{E}$ to transfer information back and forth between modular forms and derivations in $\mathfrak{u}^{\text {geom }}$.

12.1. Algebraic structure and dimensions. Let us denote the subspace of lowest weight vectors for $\mathrm{SL}_{2}$ in $\mathcal{O}\left(\mathcal{U}^{\text {geom }}\right)$ by

$$
\operatorname{lw}\left(\mathcal{O}\left(\mathcal{U}^{\text {geom }}\right)\right) \subset \mathcal{O}\left(\mathcal{U}^{\text {geom }}\right) .
$$

It is a subalgebra of $\mathcal{O}\left(\mathcal{U}^{\text {geom }}\right)$, filtered by length, and graded with respect to $M$.

On the other hand, consider

$$
\mathcal{M}_{\bullet, 0}=\bigoplus_{n \geqslant 0} \mathcal{M}_{n, 0}
$$

which defines a subalgebra of $\mathcal{M}$. It consists of functions that transform like classical modular forms (with no antiholomorphic factor of automorphy). Let us define

$$
\operatorname{lw}\left(\mathcal{M I}^{E}\right)=\mathcal{M I}^{E} \cap \mathcal{M}_{\bullet, 0},
$$


and call the elements lowest weight vectors. Although the Lie algebra $\mathfrak{s l}_{2}$ does not act on $\mathcal{M I}^{E}$ per se, the terminology is justified since images of elements in $\operatorname{lw}\left(\mathcal{M I}^{E}\right)$ behave like lowest weight vectors in the context of Section 11.3.

THEOREM 12.1. The subspace

$$
\operatorname{lw}\left(\mathcal{M I}^{E}\right) \subset \mathcal{M I}^{E}
$$

is closed under multiplication.

There is a canonical $\mathcal{Z}^{\mathrm{sv}}$-linear isomorphism of algebras

$$
\operatorname{gr}_{\bullet}^{L} \operatorname{lw}\left(\mathcal{O}\left(\mathcal{U}^{\mathrm{geom}}\right)\right) \otimes \mathcal{Z}^{\mathrm{sv}} \stackrel{\sim}{\longrightarrow} \operatorname{gr}_{\bullet}^{L} \operatorname{lw}\left(\mathcal{M I}^{E}\right) .
$$

It respects the $M$-filtration on both sides of the isomorphism.

Proof. Fix a choice of elements $\left(b^{\text {sv }}, \phi^{\text {sv }}\right)$ as in Theorem 7.2 and define $J^{\text {eqv }}(\tau)$ according to Definition 8.1. A nontrivial lowest weight vector $v \in \operatorname{lw}\left(\mathcal{O}\left(\mathcal{U}^{\text {geom }}\right)\right)$ of $\mathrm{SL}_{2}$-weight $n$ generates, under the action of $\mathrm{SL}_{2}$, an irreducible $\mathrm{SL}_{2}$-submodule

$$
c_{v}: \check{V}_{n}^{d R} \subset \mathcal{O}\left(\mathcal{U}^{\text {geom }}\right),
$$

and furthermore, every irreducible $\mathrm{SL}_{2}$-submodule arises in this way. Taking the coefficient $c_{v}\left(J^{\text {eqv }}\right)$ and extracting the term $\left(c_{v}\right)_{n, 0}$ in the manner of Proposition 9.2 defines a modular form in $\mathcal{M I}^{E}$ of modular weights $(n, 0)$. It is given explicitly by

$$
\chi(v)=\left.\mathbb{L}^{-2 n} c_{v}\left(J^{\mathrm{eqv}}\right)\right|_{X=\pi i \bar{z}, Y=\pi i} \in \mathcal{M I}^{E} \cap \mathcal{M}_{n, 0} .
$$

This extends to a $\mathcal{Z}^{\text {sv }}$-linear map

$$
\chi: \operatorname{lw}\left(\mathcal{O}\left(\mathcal{U}^{\text {geom }}\right)\right) \otimes \mathcal{Z}^{\text {sv }} \longrightarrow \operatorname{lw}\left(\mathcal{M I}^{E}\right),
$$

which respects the $M$ and $L$ filtrations. It depends on the choice of $\left(b^{\mathrm{sv}}, \phi^{\mathrm{sv}}\right)$. By the definition of $\mathcal{M I}^{E}$, every modular form of weights $(n, 0)$ arises in this way, and $\chi$ is surjective. To prove injectivity, it is enough to show that the associated graded of $\chi$ with respect to the length filtration is injective. For this, consider lowest weight vectors $v_{1}, \ldots, v_{n}$ in $L_{k} \mathcal{O}\left(\mathcal{U}^{\text {geom }}\right)$, which are linearly independent in $\operatorname{gr}_{k}^{L} \mathcal{O}\left(\mathcal{U}^{\text {geom }}\right)$. They define linearly disjoint $\mathrm{SL}_{2}$-submodules $c_{v_{1}}$, $\ldots, c_{v_{n}}$ of $\mathcal{O}\left(\mathcal{U}^{\text {geom }}\right)$. The dual of the monodromy homomorphism (6.1) defines an embedding

$$
\mathcal{O}\left(\mathcal{U}^{\text {geom }}\right) \hookrightarrow \mathcal{O}\left(\mathcal{U}_{E}^{d R}\right)=T^{c}\left(\bigoplus_{n \geqslant 1} \mathbf{E}_{2 n+2} \check{V}_{2 n}^{d R}\right)
$$


By the linear independence of iterated integrals (Corollary 4.5), the corresponding modular forms $\chi\left(v_{1}\right), \ldots, \chi\left(v_{n}\right)$ are linearly independent modulo iterated integrals of length $\leqslant k-1$, since by the remarks following Theorem 8.2, their leading terms are real and imaginary parts of iterated integrals of independent Eisenstein series. This proves injectivity.

We next show that $\chi$ is a homomorphism. Let $v_{1}, v_{2} \in \operatorname{lw}\left(\mathcal{O}\left(\mathcal{U}^{\text {geom }}\right)\right)$ of $\mathrm{SL}_{2}$ weights $n_{1}, n_{2}$. Then $c_{v_{1} v_{2}}$ is defined via the commuting diagram

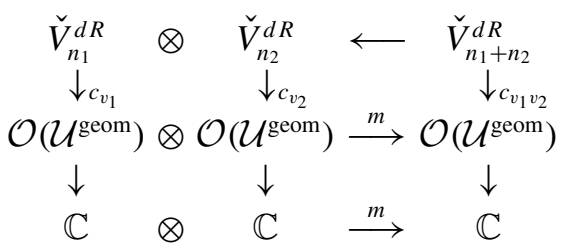

where the map along the top is dual to $\delta_{d R}^{0}: V_{n_{1}}^{d R} \otimes V_{n_{2}}^{d R} \rightarrow V_{n_{1}+n_{2}}^{d R}$, the vertical maps in the bottom square are given by the homomorphism $J^{\text {eqv }}(\tau): \mathcal{O}\left(\mathcal{U}^{\text {geom }}\right) \rightarrow \mathbb{C}$, and $m$ denotes multiplication. The reason that the top square commutes is because the corresponding square with $\mathcal{O}\left(\mathcal{U}^{\text {geom }}\right)$ replaced with $\mathcal{O}\left(\mathcal{U}_{E}^{d R}\right)$ commutes, and the monodromy map $\mu^{*}: \mathcal{O}\left(\mathcal{U}^{\text {geom }}\right) \subset \mathcal{O}\left(\mathcal{U}_{E}^{d R}\right)$ is $\mathrm{SL}_{2}$-equivariant and respects multiplication. It follows that

$$
c_{v_{1} v_{2}}\left(J^{\mathrm{eqv}}\right)=\delta_{d R}^{0}\left(c_{v_{1}}\left(J^{\mathrm{eqv}}\right) c_{v_{2}}\left(J^{\mathrm{eqv}}\right)\right) .
$$

From the definition of $\chi$, we obtain $\chi\left(v_{1} v_{2}\right)=\chi\left(v_{1}\right) \chi\left(v_{2}\right)$. Since $\chi$ is an isomorphism, this also implies that $\operatorname{lw}\left(\mathcal{M I}^{E}\right) \subset \mathcal{M} \mathcal{I}^{E}$ is stable under multiplication.

Finally, isomorphism (12.1) is obtained by replacing $\chi$ with its associated graded for the length filtration. It is well-defined since modifying $J^{\text {eqv }}$ by $J^{\text {eqv }} a$, for $a \in\left(\mathcal{U}^{\text {geom }}\right)^{\mathrm{SL}_{2}}\left(\mathcal{Z}^{\text {sv }}\right)$ changes $\chi$ by terms of lower length. This is because multiplication is trivial on the associated graded for the lower central series.

Note that the action of $\mathfrak{s l}_{2}$ on $\mathcal{O}\left(\mathcal{U}^{\text {geom }}\right)$ does not correspond to the action of the differential operators $\partial, \bar{\partial}$ on $\mathcal{M I}^{E}$.

REMARK 12.2. Note that there are elements in $\operatorname{lw}\left(\mathcal{M I}^{E}\right)$ which are polynomials in elements of $\mathcal{M I}^{E}$ of lower length, but which do not necessarily lie in $\operatorname{lw}\left(\mathcal{M I}^{E}\right)$. These relations are not captured by the previous theorem.

12.2. Orthogonality to cusp forms. It follows from Theorem 2.1 that the composite

$$
\operatorname{lw}\left(\mathcal{M I}^{E}\right) \stackrel{\partial}{\longrightarrow} \mathcal{M}_{\bullet,-1} \stackrel{p^{h}}{\longrightarrow} S,
$$


where $S$ is the complex vector space of holomorphic cusp forms, is the zero map. This sheds some light on the structure of $\mathcal{O}\left(\mathcal{U}^{\text {geom }}\right)$, and makes it clear that the generators of $\mathfrak{u}^{\text {geom }}$ have infinitely many relations coming from every cusp form.

More precisely, consider the linear map

$$
\begin{aligned}
P:\left(E \otimes \mathcal{M I}^{E}\right)_{\bullet,-1} & \longrightarrow S[\mathbb{L}] \\
\mathbb{L}^{k+1} \mathbb{G}_{a} \otimes F_{b, k} & \mapsto \mathbb{L}^{k} p^{h}\left(\mathbb{G}_{a} F_{b, k}\right),
\end{aligned}
$$

where $F_{b, k} \in \mathcal{M I}^{E}$ has modular weights $(b, k)$. Then Theorem 2.1 implies that

$$
\partial\left(\operatorname{lw}\left(\mathcal{M I}^{E}\right)\right) \subset \operatorname{ker}(P),
$$

which provides, via Theorem 12.1, a possible constraint on $\operatorname{lw}\left(\mathcal{O}\left(\mathcal{U}^{\text {geom }}\right)\right)$ for every $f \mathbb{L}^{d} \in S[\mathbb{L}]$, where $f$ is a cuspidal eigenform and $d \geqslant 0$.

12.3. Relations in $\mathfrak{u}^{\text {geom }}$ via orthogonality. Pollack's relations (Section 5.4) were initially proved in [32] in a certain quotient of $\mathfrak{u}^{\text {geom }}$ (except for the relations of depth two, which hold in $\mathfrak{u}^{\text {geom }}$ itself) by direct combinatorial computation. As a corollary of the analytic computations in [2], which rely on the RankinSelberg method, one can prove that the relations lift to $\mathfrak{u}^{\text {geom }}$. This makes heavy use of either the Tannakian formalism or Beilinson-Deligne cohomology. The reason for the existence of these relations is the presence of nontrivial extensions of the trivial motive $\mathbb{Q}$ by Tate twists of the motives of Hecke eigen cusp forms $V_{f}(w+d)$ inside the relative completion of the fundamental group of $\mathcal{M}_{1,1}$, where $w$ is the weight of $f$ and $d \geqslant 0$.

Here we suggest a much simpler conceptual explanation for these relations, which only makes use of the construction of the previous paragraph: every relation comes about because of orthogonality to cusp forms. The role of Tate twists is played by the powers of $\mathbb{L}$ on the right-hand side of (12.3). Thus, for every cuspidal Hecke eigenform $f$ and integer $d$, the term $f \mathbb{L}^{d} \in S[\mathbb{L}]$ potentially gives rise to a new relation and corresponds to an extension of $\mathbb{Q}$ by $V_{f}(w+d)$ of the kind described above.

More precisely, one has the following theorem, which, using Theorem 12.1, explains the source of relations in $\mathfrak{u}^{\text {geom }}$ from orthogonality.

THEOREM 12.3. There is an exact sequence

$$
0 \longrightarrow \operatorname{lw}\left(\mathrm{gr}_{2}^{L} \mathcal{M} \mathcal{I}^{E}\right) \stackrel{\partial}{\longrightarrow}\left(E \otimes \operatorname{gr}_{1}^{L} \mathcal{M} \mathcal{I}^{E}\right)_{\bullet,-1} \stackrel{P}{\longrightarrow} S[\mathbb{L}] .
$$

This theorem is a consequence of the results in [2]. The last map is welldefined because $L_{1} \mathcal{M} \mathcal{I}^{E} \cong \mathcal{Z}^{\text {sv }} \oplus \operatorname{gr}_{1}^{L} \mathcal{M I}^{E}$ is split. The injectivity of the first 
map follows from (2.6). Since $\mathrm{gr}_{1}^{L} \mathcal{M I}^{E}$ is generated by real-analytic Eisenstein series $\mathcal{E}_{r, s}$, the holomorphic projections can be computed [2, Section 9], using the Rankin-Selberg method, and give special values of $L$-functions of cusp forms. One deduces that the last map in the above sequence is surjective after tensoring with $\mathbb{C}$. The exactness in the middle follows from computing dimensions: the leftmost space is dual to a subquotient of $\mathfrak{u}^{\text {geom }}$ whose dimensions were computed in [32]. One has to only show that there are no other relations in length two, and the point is that it is enough to work in the same subquotient of $\mathfrak{u}^{\text {geom }}$, which is much easier than working in the full Lie algebra $\mathfrak{u}^{\text {geom }}$.

EXAMPLE 12.4. Let us illustrate with an example in weight 12 (see also [6, Example 9.8]). Let $\Delta$ denote the Ramanujan cusp form of weight 12 . The functions

$$
f_{2}=\mathbb{L} \mathbb{G}_{4} \mathcal{E}_{8,0}, \quad f_{4}=\mathbb{L} \mathbb{G}_{6} \mathcal{E}_{6,0}, \quad f_{6}=\mathbb{L} \mathbb{G}_{8} \mathcal{E}_{4,0}, \quad f_{8}=\mathbb{L} \mathbb{G}_{10} \mathcal{E}_{2,0}
$$

span $\left(E \otimes \operatorname{gr}_{1}^{L} \mathcal{M} \mathcal{I}^{E}\right)_{11,-1}$. Their holomorphic projection is given by the RankinSelberg method [2, Corollary 9.14], which shows that $\left\langle f_{2 a}, \mathbb{L} \Delta\right\rangle$ is proportional to a product of $L$-values $L(\Delta, 2 a+1) L(\Delta, 12)$, which are nonzero. By Manin's period polynomial relations, the odd critical $L$-values $L(\Delta, 2 a+1)$ for $1 \leqslant a \leqslant 4$ are proportional to each other over $\mathbb{Q}$, and one deduces that the kernel of $P$ has dimension 3. By the computations in the next paragraph, where we explicitly give the dimensions of $\mathfrak{u}^{\text {geom }}$ in low weights, we know that there exists, in addition to the two products of real-analytic Eisenstein series $\mathcal{E}_{2,0} \mathcal{E}_{8,0}$ and $\mathcal{E}_{4,0} \mathcal{E}_{6,0}$, an irreducible modular equivariant function $F \in \mathcal{M I}_{2}^{E}$. Its image under $\partial$ lies in the kernel of $P$, and satisfies

$$
\partial F=9\left(f_{2}-f_{8}\right)+14\left(f_{4}-f_{6}\right) .
$$

This equation is dual to the first of Pollack's equations (5.6) (multiply $\varepsilon_{2 n}^{\vee}$ by $(2 n-2)$ ! in (5.6) to reproduce the coefficients 9 and 14). See [2, Example 22.3], for precise details and the connection with double-shuffle equations.

REMARK 12.5. One could try to avoid the Rankin-Selberg method altogether by computing the holomorphic projection $P$ directly from the Fourier expansion using a formula of Sturm [34]. Diamantis (personal communication) has shown using Sturm's formula how to derive the first Pollack relation using the holomorphic double Eisenstein series considered in [11].

12.4. Linearized double-shuffle equations. A connection between $\mathfrak{u}^{\text {geom }}$ and linearized double-shuffle equations was described in [5]. We briefly recall the statement. 
The evaluation map (5.7), for $x=\mathrm{a}$, provides an embedding

$$
\mathfrak{u}^{\text {geom }} \hookrightarrow \operatorname{Lie}(\mathrm{a}, \mathrm{b}) \subset T^{c}(\mathrm{a}, \mathrm{b}) .
$$

The tensor coalgebra $T^{c}(\mathrm{a}, \mathrm{b})$ is graded by the degree in $\mathrm{b}$. Consider the linear map

$$
\begin{aligned}
\rho: \operatorname{gr}_{\mathrm{b}}^{r} T^{c}(\mathrm{a}, \mathrm{b}) & \longrightarrow \mathbb{Q}\left(x_{1}, \ldots, x_{r}\right) \\
\mathrm{a}^{i_{0}} \mathrm{ba}^{i_{1}} \mathrm{~b} \ldots \mathrm{ba}^{i_{r}} & \mapsto \frac{x_{1}^{i_{1}} \ldots x_{r}^{i_{r}}}{x_{1}\left(x_{1}-x_{2}\right) \ldots\left(x_{r-1}-x_{r}\right) x_{r}}
\end{aligned}
$$

for $r \geqslant 1$. The space $\mathfrak{p l s}=\bigoplus_{r \geqslant 1} \mathfrak{p l s}^{r}$ was defined in [5] to be a bigraded Lie algebra of homogeneous rational functions $f$ in the variables $x_{1}, \ldots, x_{r}$, for all $r \geqslant 2$ with the property

$$
x_{1}\left(x_{1}-x_{2}\right) \ldots\left(x_{r-1}-x_{r}\right) x_{r} f \in \mathbb{Q}\left[x_{1}, \ldots, x_{r}\right],
$$

which satisfy the linearized double-shuffle equations. It is equipped with a version of the linearized Ihara Lie bracket, which involves symmetrization of rational functions over the action of a dihedral group. The space $\mathfrak{p l s}^{2}$ is described by the solutions to

$$
\begin{array}{r}
f\left(x_{1}, x_{2}\right)+f\left(x_{2}, x_{1}\right)=0 \\
f\left(x_{1}, x_{1}+x_{2}\right)+f\left(x_{2}, x_{1}+x_{2}\right)=0
\end{array}
$$

and for $r=3$, these equations take the form

$$
\begin{array}{r}
f\left(x_{1}, x_{2}, x_{3}\right)+f\left(x_{2}, x_{1}, x_{3}\right)+f\left(x_{2}, x_{3}, x_{1}\right)=0 \\
f\left(x_{1}, x_{12}, x_{123}\right)+f\left(x_{2}, x_{12}, x_{123}\right)+f\left(x_{2}, x_{23}, x_{123}\right)=0,
\end{array}
$$

where $x_{i j}=x_{i}+x_{j}$ and $x_{123}=x_{1}+x_{2}+x_{3}$. The space $\mathfrak{p l s}$ is in addition equipped with an action of $\mathfrak{s l}_{2}$.

THEOREM 12.6 [5]. The linear map $\rho$, when restricted to $\mathfrak{u}^{\text {geom }}$, defines an injective map of bigraded Lie algebras

$$
\rho: \mathfrak{u}^{\text {geom }} \longrightarrow \mathfrak{p l s},
$$

which commutes with the action of $\mathfrak{s l}_{2}$ on both sides.

By Theorem 12.1, we deduce a canonical surjection

$$
\operatorname{gr}_{L}^{\bullet} \operatorname{lw}(\mathcal{O}(\mathcal{P})) \otimes \mathcal{Z}^{\mathrm{sv}} \longrightarrow \operatorname{gr}_{L}^{\bullet} \operatorname{lw}\left(\mathcal{M I}^{E}\right),
$$


where $\mathcal{P}$ denotes the affine group scheme corresponding to the graded Lie algebra $\mathfrak{p l s}$.

The b-degree on $\mathfrak{u}^{\text {geom }}$ coincides with the grading $r$ on $\mathfrak{p l s}$. In [5], we proved the following theorem.

THEOREM 12.7. The map $\rho: \mathfrak{u}^{\text {geom }} \rightarrow \mathfrak{p l s}$ is an isomorphism in $\mathrm{b}$-degrees $\leqslant 3$. If

$$
u_{k}(s)=\sum_{n \in \mathbb{Z}} s^{n} \operatorname{dim}_{\mathbb{Q}}\left(\operatorname{gr}_{k}^{B} \operatorname{gr}_{2 n}^{M} \mathfrak{u}^{\text {geom }}\right)
$$

denotes its Poincaré series, then

$u_{1}(s)=\frac{s}{1-s^{2}}, \quad u_{2}(s)=\frac{s^{2}}{\left(1-s^{2}\right)\left(1-s^{6}\right)}, \quad u_{3}(s)=\frac{s}{\left(1-s^{2}\right)\left(1-s^{4}\right)\left(1-s^{6}\right)}$.

Note that in [5], we included $\varepsilon_{0}$ in the definition of $\mathfrak{u}^{\text {geom }}$, which led to a marginally different formula for $u_{1}(s)$.

REMARK 12.8. The bigraded Lie subalgebra

$$
\mathfrak{L}=\bigoplus_{r \geqslant 1} \mathfrak{p l s} \cap \mathbb{Q}\left[x_{1}, \ldots, x_{r}\right]
$$

of solutions to linearized double-shuffle equations in polynomials (that is, with no poles) is related to the structure of depth-graded motivic multiple zeta values $[3,24]$. It follows from Theorem 12.1 that the structure of $\mathcal{M I}^{E}$ is intimately connected to the structure of depth-graded multiple zeta values. It would be interesting to compare with [17].

\section{3. $L$-functions associated with modular forms in $\mathcal{M} \mathcal{I}^{E}$}

Hecke associated an $L$-function with every classical holomorphic modular form. One can do the same for functions in $\mathcal{M I}^{E}$.

Definition 13.1. Let $f \in \mathcal{M I}^{E}$ and let $f^{0}$ denote its constant part. Its completed $L$-function is defined for $\operatorname{Re}(s)$ large by the regularized Mellin transform:

$$
\Lambda(f ; s)=\int_{0}^{\infty}\left(f(i y)-f^{0}(i y)\right) y^{s} \frac{d y}{y} .
$$

The integral converges more precisely for $\operatorname{Re}(s)>w+k$, where $w$ is the weight of $f$ and $k$ is the maximum of 0 and the largest $k$ such that the coefficient $a_{0,0}^{(k)}(f)$ is nonzero. This follows from (13.1) and Theorem 10.6, which implies that $a_{0,0}^{(k)}(f)=0$ for $k<-w$. 
The definition applies to a much more general class of modular functions [6, Section 9.4].

Proposition 13.2. If $f$ has modular weights $(\alpha, \beta)$, the function $\Lambda(f ; s)$ admits a meromorphic continuation to $\mathbb{C}$ and satisfies the functional equation

$$
\Lambda(f ; s)=i^{h} \Lambda(f ; w-s),
$$

where $w=\alpha+\beta$ and $h=\alpha-\beta$. It has at most simple poles of the form

$$
\sum_{k}(-2 \pi)^{k} a_{0,0}^{(k)}\left(\frac{i^{h}}{s-w-k}-\frac{1}{s+k}\right),
$$

where $f^{0}=\sum_{k} a_{0,0}^{(k)} \mathbb{L}^{k}$ is the constant part of $f$. Furthermore, it can be expressed in terms of Dirichlet series as follows. Let

$$
L^{(k)}(f ; s)=\sum_{\substack{m, n \geqslant 0 \\ m+n \geqslant 1}} \frac{a_{m, n}^{(k)}}{(m+n)^{s}},
$$

where $a_{m, n}^{(k)}$ are the expansion coefficients of (1.4) of $f$. Then

$$
\Lambda(f ; s)=\sum_{k}(-1)^{k}(2 \pi)^{-s} \Gamma(s+k) L^{(k)}(f ; s+k) .
$$

Since the functions in $\mathcal{M I}^{E}$ are naturally associated with universal mixed elliptic motives, their $L$-functions are liable to contain interesting arithmetic information. We do not know the values of $\Lambda(f ; s)$ for integers $s>w$, even when $f$ is a product of two real-analytic Eisenstein series.

\subsection{Compatibility with the differential structure}

LEMMA 13.3. The following relation holds for all $s \in \mathbb{C}$, where $w=\alpha+\beta$ :

$$
\Lambda(\partial f ; s)+\Lambda(\bar{\partial} f ; s)+(2 s-w) \Lambda(f ; s)=0 .
$$

Proof. Set $z=i y$, where $y \in \mathbb{R}$. For $\operatorname{Re}(s)$ sufficiently large, take the regularized Mellin transform from 0 to $\infty$ (see [1] for the definition) of both sides of the following equation, which follows from the definition of $\partial_{\alpha}, \bar{\partial}_{\beta}$ :

$$
d\left(f y^{s}\right)=\left(y \frac{\partial f}{\partial y}+s f\right) y^{s-1} d y=\frac{1}{2}\left(\partial_{\alpha} f+\bar{\partial}_{\beta} f-w f\right) y^{s-1} d y+s f y^{s-1} d y .
$$


Conclude by analytic continuation for all $s \in \mathbb{C}$. Alternatively, one can simply apply [6, Lemma 2.6], which relates the expansion coefficients $a_{m, n}^{(k)}$ of $f, \partial f$ and $\bar{\partial} f$, and substitute into the formula for the Dirichlet series $L^{(k)}(\bullet, s)$.

EXAMPLE 13.4. Using Lemma 13.3 and the differential equations (Section 2.5) for $\mathcal{E}_{\alpha, \beta}$, one obtains equations which one can solve to express the $L$-functions of real-analytic Eisenstein series in terms of Hecke $L$-functions of holomorphic Eisenstein series. This follows from the proof of the theorem 13.5. More precisely, one finds that

$$
\Lambda\left(\mathcal{E}_{\alpha, \beta} ; s\right)=\frac{\pi P_{\alpha, \beta}(s)}{s(s-2) \ldots(s-w-2)(s-w)} \Lambda\left(\mathbb{G}_{w+2} ; s+1\right),
$$

where $w=\alpha+\beta$, and $P_{\alpha, \beta}(s) \in \mathbb{Q}[s]$ has degree less than $w / 2$ and satisfies

$$
P_{\alpha, \beta}(w-s)=P_{\alpha, \beta}(s) .
$$

For any $f$ with real Fourier coefficients, $\Lambda(\bar{f} ; s)=\Lambda(f ; s)$. Therefore $\Lambda\left(\mathcal{E}_{\alpha, \beta} ; s\right)=\Lambda\left(\mathcal{E}_{\beta, \alpha} ; s\right)$ and so $P_{\alpha, \beta}$ is symmetric in $\alpha, \beta$. The function $\Lambda\left(\mathcal{E}_{\alpha, \beta} ; s\right)$ has simple poles at $s=-1, w+1$ with rational residues, and at $0, w$ with residues proportional to $\zeta(w+1)$. Some examples are

$$
\begin{array}{r}
\Lambda\left(\mathcal{E}_{2,0} ; s\right)=\Lambda\left(\mathcal{E}_{0,2} ; s\right)=\frac{(s-1) \pi}{s(s-2)} \Lambda\left(\mathbb{G}_{4}, s+1\right) \\
\Lambda\left(\mathcal{E}_{1,1} ; s\right)=\frac{-2 \pi}{s(s-2)} \Lambda\left(\mathbb{G}_{4}, s+1\right),
\end{array}
$$

where $\Lambda\left(\mathbb{G}_{2 n} ; s\right)=(2 \pi)^{-s} \Gamma(s) \zeta(s) \zeta(s-2 n+1)$. In general, the middle term $P_{k, k} \in \mathbb{Q}$ is constant. In fact, we claim that one obtains

$$
(-4 \pi)^{k} \Lambda\left(\mathcal{E}_{k, k} ; s\right)=\frac{(2 k-1) !}{(k-1) !} \xi(s+1) \xi(s-2 k),
$$

where $\xi(s)=\pi^{-s / 2} \Gamma(s / 2) \zeta(s)$ is the completed Riemann zeta function.

13.2. Relation between nonholomorphic and mixed $\boldsymbol{L}$-functions. In [1], we defined $L$-functions in several variables as iterated Mellin transforms of theta functions.

THEOREM 13.5. Let $F \in \mathcal{M I}_{\ell}^{E}$ have modular weights $\alpha, \beta \geqslant 0$, with $w=\alpha+\beta$. Then

$$
s(s-1) \ldots(s-w) \Lambda(F ; s)
$$


is a $\mathcal{Z}^{\mathrm{sv}}[\pi, s]$-linear combination of the mixed L-functions defined in [1]:

$$
\Lambda\left(f_{1}, \ldots, f_{k} ; a_{1}, \ldots, a_{k-1}, a_{k}+s\right),
$$

where $k \leqslant \ell, f_{1}, \ldots, f_{k}$ are holomorphic Eisenstein series, and $a_{1}, \ldots, a_{k}$ are integers such that $1 \leqslant a_{i} \leqslant$ weight $\left(f_{i}\right)-1$ for all $1 \leqslant i \leqslant k-1$.

Proof. By Theorem 11.3, $\mathcal{M I}_{k}^{E}$ is generated by families of functions $F_{\alpha, \beta}$ satisfying

$$
\begin{aligned}
& \partial F_{\alpha, \beta}-(\alpha+1) F_{\alpha+1, \beta-1} \in E \times \mathcal{M I}_{k-1}^{E} \\
& \bar{\partial} F_{\alpha, \beta}-(\beta+1) F_{\alpha-1, \beta+1} \in \bar{E} \times \mathcal{M I}_{k-1}^{E},
\end{aligned}
$$

where we write $F_{-1, *}=F_{*,-1}=0$, and $E$ denotes the graded $\mathbb{Q}[\mathbb{L}]$-vector space generated by holomorphic Eisenstein series. Let $\alpha+\beta=w$ and write $\Lambda_{\alpha, \beta}=$ $\Lambda\left(F_{\alpha, \beta} ; s\right)$ and $\Lambda_{-1, *}=\Lambda_{*,-1}=0$. Lemma 13.3 implies that

$$
(\alpha+1) \Lambda_{\alpha+1, \beta-1}+(2 s-w) \Lambda_{\alpha, \beta}+(\beta+1) \Lambda_{\alpha-1, \beta+1} \equiv 0
$$

modulo regularized Mellin transforms of elements in $(E+\bar{E}) \times \mathcal{M I}_{k-1}^{E}$. We first need to check that this system of equations can be solved for the $\Lambda_{\alpha, \beta}$. This is obviously true for generic $s$ since the determinant of this system of linear equations defines a polynomial in $s$ and has finitely many zeros. In fact, one easily verifies that the matrix

$$
M_{w}=\left(\begin{array}{cccccc}
2 s-w & 1 & & & & \\
w & 2 s-w & 2 & & & \\
& w-1 & 2 s-w & 3 & & \\
& & \ddots & \ddots & & \\
& & & 2 & 2 s-w & w \\
& & & & 1 & 2 s-w
\end{array}\right)
$$

has determinant $\operatorname{det}\left(M_{w}\right)=2^{w+1} s(s-1) \ldots(s-w)$. To see this, note that $M_{w}$ is the matrix of $D+(2 s-w)$, where $D=x \frac{\partial}{\partial y}+y \frac{\partial}{\partial x}$ acting on the space $\bigoplus_{i+j=w} x^{i} y^{j} \mathbb{C}$ of homogeneous polynomials of degree $w$. The eigenvectors for $D$ are the set of polynomials $(x+y)^{i}(x-y)^{j}$, where $i+j=w$, and have eigenvalue $i-j$. Therefore $\operatorname{det}\left(M_{w}\right)$ vanishes at $w-2 s=i-j$, or equivalently, $s=j$, for $j=0, \ldots, w$. A row expansion of $M_{w}$ shows that the leading coefficient of $s$ in $\operatorname{det}\left(M_{w}\right)$ is $2^{w+1}$.

We deduce that

$$
s(s-1) \ldots(s-w) \Lambda\left(F_{\alpha, \beta} ; s\right)
$$


is a $\mathcal{Z}^{\mathrm{sv}}[\pi, s]$-linear combination of regularized Mellin transforms [1]

$$
\int_{0}^{\overrightarrow{1}_{\infty}} f(i y) \mathcal{A}(i y) y^{s+p} \frac{d y}{y}
$$

and their complex conjugates, where $f \in E, \mathcal{A} \in \mathcal{M I}_{k-1}^{E}, p \in \mathbb{N}$. By the definition of $J^{\text {eqv }}$, the restriction of $\mathcal{A}$ to the imaginary axis can be expressed as a linear combination over $\mathcal{Z}^{\mathrm{sv}}[\pi, s]$ of regularized iterated integrals of length at most $k-1$ of holomorphic Eisenstein series $f_{i}(\tau) \tau^{r_{i}}$, where $0 \leqslant r_{i} \leqslant n_{i}-2$ if $f_{i}$ is of weight $n_{i}$, since complex conjugation introduces at most a sign. Substituting into (13.2) gives exactly the quantity $\Lambda\left(f_{1}, \ldots, f_{k-1}, f ; r_{1}, \ldots, r_{k-1}, p+s\right)$.

This result holds for more general modular iterated integrals, but can also be made more precise in this situation: indeed, the linear combination in the statement of the theorem is effectively computable.

\section{Acknowledgements}

This project has received funding from the European Research Council (ERC) under the European Union's Horizon 2020 research and innovation programme (grant agreement no. 724638) and was written during a stay at the IHES. Many thanks are due to Richard Hain for ongoing discussions about relative completion and mixed elliptic motives and to Nils Matthes, for corrections. Many thanks also go to Andrey Levin, who suggested several years ago that double elliptic polylogarithms could be orthogonal to cusp forms.

\section{Conflict of Interest: None.}

\section{References}

[1] F. Brown, A multi-variable version of the completed Riemann zeta function and other $L$ functions, https://arxiv.org/abs/1904.00190.

[2] F. Brown, Multiple modular values and the relative completion of the fundamental group of $M_{1,1}$, arXiv:1407.5167.

[3] F. Brown, Depth-graded motivic multiple zeta values, arXiv:1301.3053.

[4] F. Brown, 'Polylogarithmes multiples uniformes en une variable', C. R. Math. Acad. Sci. Paris 338(7) (2004), 527-532.

[5] F. Brown, 'Zeta elements in depth 3 and the fundamental Lie algebra of the infinitesimal Tate curve', Forum Math. Sigma 5 (2017), doi:10.1017/fms.2016.29, arXiv:1504.04737.

[6] F. Brown, 'A class of non-holomorphic modular forms I', Res. Math. Sci. 5 (2018), 7, https:// doi.org/10.1007/s40687-018-0130-8, (https://arxiv.org/abs/1710.07912). 
[7] F. Brown, 'A class of non-holomorphic modular forms III: real analytic cusp forms', Res. Math. Sci. 5 (2018), 34, https://doi.org/10.1007/s40687-018-0151-3, (https://arxiv.org/abs/17 10.07912).

[8] F. Brown and A. Levin, Multiple elliptic polylogarithms, arXiv:1110.6917.

[9] D. Calaque, B. Enriquez and P. Etingof, 'Universal KZB equations: the elliptic case', in Algebra, Arithmetic, and Geometry: in honour of Yu. I. Manin, Vol. I, Progress in Mathematics, 269 (Springer, Birkhäuser Boston, 2009), 165-266.

[10] K. T. Chen, 'Iterated path integrals', Bull. Amer. Math. Soc. 83 (1977), 831-879.

[11] N. Diamantis and C. O'Sullivan, 'Kernels for products of L-functions', Algebra Number Theory 7(8) (2013), 1883-1917.

[12] E. D'Hoker and M. Green, 'Identities between modular graph forms', J. Number Theory 189 (2018), 25-88.

[13] E. D’Hoker, M. B. Green, O. Gürdogan and P. Vanhove, Modular graph functions, arXiv:1512.06779.

[14] E. D'Hoker and J. Kaidi, 'Hierarchy of modular graph identities', J. High Energy Phys. (11)051 (2016).

[15] M. Eichler, 'Eine Verallgemeinerung der Abelschen Integrale', Math. Z. 67 (1957), 267-298.

[16] B. Enriquez, 'Elliptic associators', Selecta Math. (N.S.) 20(2) (2014), 491-584.

[17] H. Gangl, M. Kaneko and D. Zagier, 'Double zeta values and modular forms', in Automorphic Forms and Zeta Functions (World Sci. Publ., Hackensack, NJ, 2006), 71-106.

[18] M. Green, J. Russo and P. Vanhove, 'Low energy expansion of the four-particle genus-one amplitude in type II superstring theory', J. High Energy Phys. 2008 (2008), doi:10.1088/112 6-6708/2008/02/020.

[19] R. Hain, 'Notes on the universal elliptic KZB equation', Pure Appl. Math. Q. 12(2) (2016), International Press, [arXiv:1309.0580].

[20] R. Hain, Ihara curves, unpublished notes.

[21] doi:10.1017/S1474748018000130 R. Hain and M. Matsumoto, 'Universal mixed elliptic motives', J. Inst. Math. Jussieu 19(3) 663-76610.1017/S1474748018000130.

[22] R. Hain, 'The Hodge de Rham theory of the relative Malcev completion', Ann. Sci. Éc. Norm. Supér. 31 (1998), 47-92.

[23] R. Hain, 'The Hodge-de Rham theory of modular groups', in Recent Advances in Hodge Theory, London Mathematical Society Lecture Note Series, 427 (Cambridge University Press, Cambridge, 2016), 422-514.

[24] K. Ihara, M. Kaneko and D. Zagier, 'Derivation and double shuffle relations for multiple zeta values', Compos. Math. 142 (2006), 307-338.

[25] A. Levin and G. Racinet, Towards multiple elliptic polylogarithms, arXiv:math/0703237.

[26] M. Kontsevich and D. Zagier, 'Periods', in Mathematics Unlimited- 2001 and Beyond (Springer, Berlin, 2001), 771-808.

[27] Y. Manin, 'Periods of parabolic points and p-adic Hecke series', Math. Sb. (1973), 371-393.

[28] Y. Manin, 'Iterated Shimura integrals', Moscow Math. J. 5 (2005), 869-881.

[29] Y. Manin, 'Iterated integrals of modular forms and non-commutative modular symbols', in Algebraic Geometry and Number Theory, Progress in Mathematics, 253 (Springer, Birkhäuser Boston, 2006), 565-597.

[30] N. Matthes, 'On the algebraic structure of iterated integrals of quasimodular forms', Algebra Number Theory 11(9) (2017), 2113-2130.

[31] H. Nakamura, 'Tangential base points and Eisenstein power series', in Aspects of Galois Theory, London Mathematical Society Lecture Note Series, 256 (Cambridge University Press, Cambridge, 1999), 202-217. 
[32] A. Pollack, Relations between derivations arising from modular forms, undergraduate thesis, Duke University, (2009).

[33] G. Shimura, 'Sur les intégrales attachées aux formes automorphes', J. Math. Soc. Japan 11 (1959), 291-311.

[34] J. Sturm, 'Projections of $C^{\infty}$ automorphic forms', Bull. Amer. Math. Soc. (N.S.) 2(3) (1980), 435-439.

[35] H. Tsunogai, 'On some derivations of Lie algebras related to Galois representations', Publ. Res. Inst. Math. Sci. 31 (1995), 113-134.

[36] D. Zagier, 'The 1-2-3 of modular forms', in Elliptic Modular Forms and Their Applications, Universitext (Springer, Berlin, 2008), 1-103.

[37] F. Zerbini, 'Single-valued multiple zeta values in genus 1 superstring amplitudes', Commun. Number Theory Phys. 10(4) (2016), 703-737. 